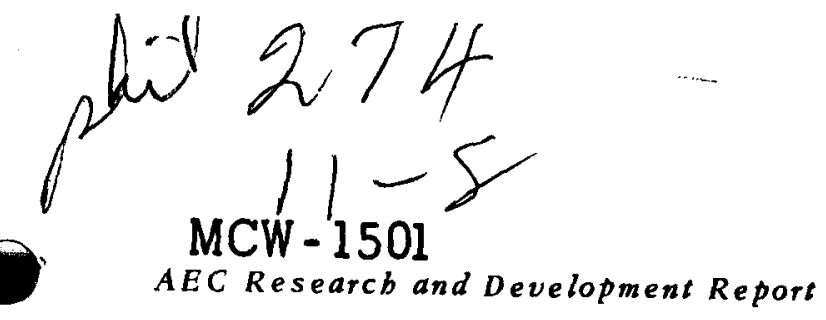

\title{
SOME OBSERVATIONS ON THE ELECTRON METALLOGRAPHY OF URANIUM
}

BELEASED FOR ANNOUNCEMENT

IN NUCLEAR SCIENCE ABETRACTS

August 1, 1966
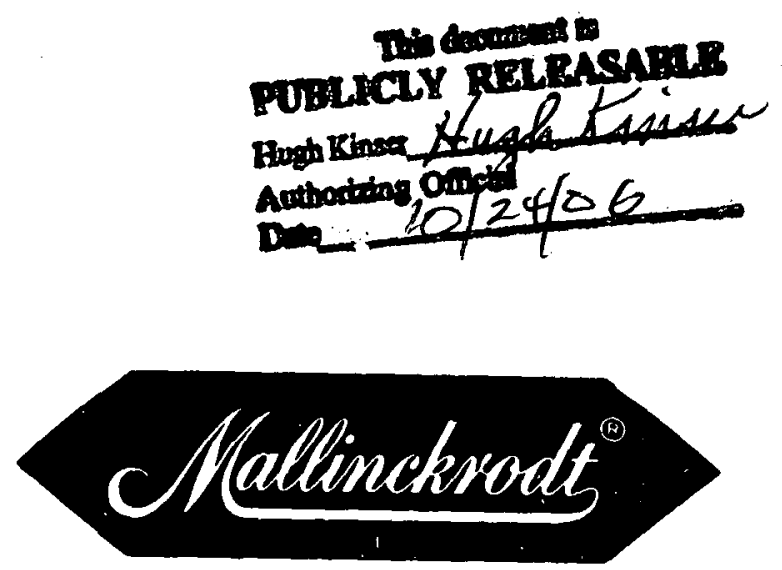

WELDON SPRING PLANT

\author{
MALLINCKRODT OHEMICAL WORKS \\ URANIUM DIVISION \\ Contract No. W-14-108-Eng-8
}




\section{DISCLAIMER}

This report was prepared as an account of work sponsored by an agency of the United States Government. Neither the United States Government nor any agency Thereof, nor any of their employees, makes any warranty, express or implied, or assumes any legal liability or responsibility for the accuracy, completeness, or usefulness of any information, apparatus, product, or process disclosed, or represents that its use would not infringe privately owned rights. Reference herein to any specific commercial product, process, or service by trade name, trademark, manufacturer, or otherwise does not necessarily constitute or imply its endorsement, recommendation, or favoring by the United States Government or any agency thereof. The views and opinions of authors expressed herein do not necessarily state or reflect those of the United States Government or any agency thereof. 


\section{DISCLAIMER}

Portions of this document may be illegible in electronic image products. Images are produced from the best available original document. 


\section{LEGAL NOTICE}

This report was prepared as an account of Government sponsored work. Neither the United States, nor the Commission, nor any person acting on behalf of the Commission:

A. Makes any warranty or representation, expressed or implied, with respect to the accurocy, completeness, or usefulness of the information contained in this repart, or that the use of any information, apparatus, method, or process disclosed in this report may not infringe privately owned rights; or

B. Assumes any liabilities with respect to the use of, or for domages resulting from the use of any information, apparatus, method, or process disclosed in this report.

As used in the above, "person acting on behalf of the Commission" includes any employee or controctor of the Commission, or employee of such controctor, to the extent that such employee or contractor of the Commission, or employee of such contractor prepares, disseminates, or provides access to, any information pursuant to his omployment or contract with the Commission, or his employment with such contractor.

Printed in USA. Price $\$ 3.00$. Available from the Clearinghouse for Federal Scientific and Technical Information, National Bureau of Standards, U. S. Department of Commerce, Springfield, Virginia 
MCW-1501

UC-25, Metals, Ceramics, and Materials (TID-4500, 48th Edition)

by

H. C. Kloepper, Jr.
BETEASED FOR ALROUNGEAENT

IN TUCLAAR SGIENCE ABSTRACTS

August 1, 1966

\author{
Mallinckrodt Chemical Works \\ Uranium Division \\ Weldon :Spring, Missouri
}


Number of Copies

Interna1

12

External in accordance with TID-4500, 48th Edition

Category UC-25, Metals, Ceramic8, and Materials

Special

Battelle-Northwest

J. E. Minor

R. S. Kemper

F. W. Albaugh

S. H. Bush

J. J. Cadwe11

G. A. Last

Cincinnati Area Office U.S.A.B.C.

C. L. $\operatorname{Rar} 1$

W. T. Warner

Division of Production, Washington U.S.A.E.C.

F. P. Baranowski

1

F. R. Dowling

W. Devine, Jr.

Douglas United Nuclear, Richland

W. M. Mathis

J. T. Stringer

C. D. Harrington

C. W. Kuhlman

Dow Chemical Co., Rocky Flats

L. A. Matheson

du Pont Company, Aiken

T. C. Evans

R. T. Huntoon

1

W. R. McDonnell

du Pont Company, Wilmington

W. B. Delong :

J. W. Croach 
General Electric Company, Richland

M. Lewis

J. W. Nicholaus

A. E. Guay

T. D. Naylor

1

1

1

1

National Lead Company of Ohio

S. Marshall

C. E. Pokon

C. B. Bussert

1

1

1

Oak Ridge Operations Office

S. A. Sapirie

J. W. Ruch

B. M. Robinson

R. E. Wedemeyer

1

1.

1

1

Richland Operations office

A. T. Gifford

P. M. Midkiff

O. W. Rathbun

1

1

St. Louis Area Office

F. H. Belcher

1

Savannah River Operations office

H. L. Kilburn

I. A. Hobbs

N. J. Donahue 
SOME OBSERVATIONS ON THE ELECTRON METALLOGRAPHY OF URANIUM

by

H. C. Kloepper, Jr.

\begin{abstract}
In this report, metallographic techniques are described that are necessary in the preparation of thin-foil specimens of uranium metal that are sultable for study at high magnification with the transmission electron microscope. Also discussed are the relationships between structure revealed in electron microscopy and $x$-ray diffraction data generated from the same area by the electron beam. Appropriate interpretation of such data permits the identification of the orientation of the matrix lattice and the determination of the crystallographic sites at which dislocations accumulate and precipitates originate. The procedures also reveal the shape and orientation of precipitate particles within the matrix. Application of these techniques to three beta-quenched, alpha-annealed U-Si alloys disclosed that precipitation of $\mathrm{U}_{3} \mathrm{Si}$ did not occur within reasonable annealing times at $500^{\circ} \mathrm{C}$ or 1 ess but that precipitation readily took place at dislocation sites when annealed at $600^{\circ} \mathrm{C}$, the number and size of particles increasing with annealing time. The $\mathrm{U}_{3} \mathrm{Si}$ particles were needle-shaped with a preferred orientation to the uranium matrix. Other studies of structures of a U-Si alloy after tensile testing indicated that the deformation at room temperature was by twinning whereas at $500^{\circ} \mathrm{C}$ the metallic flow was mainly by slip.
\end{abstract}


SOME OBSERVATIONS OF THE ELECTRON METALLOGRAPHY OF URANIUM

by

H. C. Kloepper, Jr.*

\section{INTRODUCTION}

The long range object of this work was to investigate the effect of solidsolution alloys, of two-phase alloy systems, and of strain rate on the stress necessary to initiate and continue dislocation movement in uranium structures, since this has been ${ }_{1}$ postulated to be intimately connected with tendencies for irradiation growth. Since the formation, habit, and stability at elevated temperatures of second-phase particles have been believed to be important in influencing dislocation movement and therefore a factor contributing to the stability of a fuel element under irradiation, a study of such characteristics of uranium alloys was scheduled with the electron microscope. Uraniumsilicon alloys were chosen for the first study since they had shown the most promise in resisting grain-boundary tearing during irradiation.

The short range prerequisite for this work was to acquire a familiarization with the techniques of sample preparation, microscope calibration and manipulation, and with the crystallography and crystallographic manipulations. necessary to undertake the long-range objectives briefly outlined above. This paper will primarily be concerned with experimental techniques although several examples of preliminary findings relating to the longer-range objectives will be reported.

\section{TECHNIQUES IN ELECTRON MICROSCOPY}

\section{Sample Preparation}

The description of specimen preparation for this work will deal with the preparation of thin sections from bulk samples derived from heat-treated fuel core sections from portions of tensile test specimens. The reason for this lies in the hope of correlating the electron microscope observations with bulk properties.

Slices 10-20 mils thick should be cut from bulk specimens using a $1 / 32-$ inchthick 60 grit silicon carbide wheel. The sample should be generously flooded with water and cutting should be performed slowly to minimize any heating from the abrasive action. The thickness of the rough specimen should be measured

* Now associated with Mallinckrodt Chemical Works, St. Louis, Missouri. 
so that the grinding and polishing sequences may be adjusted to yield a final foil specimen from the center of the original sample. The slice should then be sealed to a 1 1/4-inch-diameter stainless steel mount with Fisher High Temperature Seating Cement and the thickness of the combined specimen and mount measured.

A group of such stainless steel mounts may be put into the holder of a Buehler Automet polisher. The specimens should be ground wet on successively finer grit silicon carbide papers starting with 180 grit paper and finishing with 600 grit paper. Grinding times should be adjusted to remove a layer approximately 2 mils less than half of the specimen thickness. The specimen should then be polished on an $A B$ Microcloth covered wheel charged with Linde $A$ and chromic acid and finally on an $A B$ Microcloth covered wheel charged with Linde $B$ and chromic acid. If optical photo-micrographs are to be obtained, the sample may be electropolished and photographed at this point.

After photographing, the specimen and mount should be immersed in benzene to dissolve the cement holding the specimen to the mount. The thickness of the cleaned specimen should then be carefully measured and the polished face of the specimen cemented to the mount and the specimen and mount thickness again measured. The grinding and polishing steps should then be repeated. This time, however, the grinding should be continued until the specimen is 4 mils thick. As a quick visual guide to the specimen thickness remaining, the sample mounts have been provided with a -mil depression in the center. Thus when metal begins to be removed from the edges of the mount, it is time to check the specimen thickness carefully and then continue grinding cautiously until the desired thickness value is reached.

The polished specimen should then be removed from the holder, thoroughly cleaned, and finally sectioned into a number of $1 / 8$ inch-diameter disks with a specimen screen punch. The disks may be prepared for electrolytic thinning by securing each in turn in a clamping type stainless steel tweezer. Before thinning can proceed, the edges and one side of the specimen as well as the tip of the tweezer should be coated with lacquer and allowed to dry thoroughly. Polystyrene pellets dissolved in benzene makes a suitable stop-off lacquer.

A sample may then be thinned electrolytically using a modification of the thinning technique of $\mathrm{J}$. 0 . Stiegler for copper specimens devised at the Oak Ridge National Laboratory. The apparatus may consist of a glass flask similar in construction to a wash bottle. In the exit tube a platinum wire should be sealed and beyond this the tube should be constricted to form a nozzle. The electrolyte should be forced through a 20 -mil opening in the nozzle under about 1 to 2 psi of air pressure. The electrolyte stream completes the electric circuit between the platinum cathode and the uranium anode, which should be at 80 volts potential under open circuit conditions. The electrolyte should consist of 75.8 of $\mathrm{CrO}_{3}$ dissolved in $42 \mathrm{mi}$. of ater and $400 \mathrm{ml}$. of glacial acetic acid and may be used at temperatures up to room temperature. 
The action of the small stream of electrolyte should be such that a dimple will be formed where the stream hits the uranium surface. The sample may be dimpled from one or from both faces of the specimen. If both faces are to be dimpled, only the edges of the specimen need to be lacquered. The dimpling procedure should continue until a small hole in the sample appears. At this point the thinning should immediately be stopped and the sample thoroughly rinsed as quickly as possible.

After cleaning away the stop-off lacquer with benzene, the specimen should be rinsed with acetone and dried. The specimen should then be cleansed of oxide electrolytically, using a solution consisting of $75 \mathrm{ml}$. of sulfuric acid, $7 \mathrm{ml}$. of water and $18 \mathrm{ml}$. of glycerin at $35^{\circ}$ to $40^{\circ} \mathrm{F}$. An open-circuit potential of 10 volts should be used between the platinum cathode and the specimen. Since transfer time to the microscope after cleaning is especially critical to minimize reoxidation, stop-off lacquer should not be used. Instead, the edge of the sample should be held gingerly between the tweezers connected to the power supply. The sample should be immersed in the electrolyte only far enough for the surface tension to cause the dimple to be covered but not the tweezers. A green film will quickly form on the specimen, indicating that the cleanning action has commenced. The specimen may be immersed for as much as 30 seconds after the green film appears without excessive loss of specimen area. The specimen should be removed from the electrolyte with the current on and quickly plunged into water and agitated to minimize chemical attack. The sample should first be quickly washed with water and then with alcohol, drying thereafter with a blast from. a Freon gas duster. The specimen should then be placed immediately in the sample holder and transferred to the microscope. There is no need to place the specimen on a copper grid as the dimpled specimen is capable of supporting itself. If removal of oxide was not complete or if oxidation has occurred in the microscope, the specimen should be cleaned again. Only a limited number of cleanings are possible, however, before excessive loss of thin-foil area occurs.

Galibration of the Electron Microscope

In order to use effectively transmission electron microscopy as a tool for the study of metallic structure, the microscope must be calibrated for magnification, rotation, and diffraction... Magnification calibration is necessary to determine accurately the size of all features observed in electron micrographs and may be conveniently determined with a replica from a 15000-1ine-per-inch diffraction grating.:

Rotation and diffraction calibrations are especially important as these two permit the correlation of features in the photomicrograph with crystal orientations. Rotation calibration may be easily accomplished using sublimed $\mathrm{MoO}_{3}$ crystals. These crystals grow as thin, long, flat needles such that the long dimension is the [100] direction, the width is the [001] direction and the thickness is the [010] direction. By photographing a crystal at various magnification and superimposing the selected area diffraction pattern, one can 
measure the angle between the [100] direction in the crystal and the [100] direction in the diffraction pattern. This angle will vary with the magnification.

Diffraction calibration can be combined with the magnification calibration if the replica is shadowed with gold, platinum or other heavy metal. Diffraction patterns can be obtained from this replica and the appropriate diffraction constants calculated from the measured lines on the photographic plate.

\section{Crystallographic Data}

In order to interpret the data that are available in the micrographs and the corresponding diffraction patterns, recourse must be made to the crystal = lography of the materials being investigated. It is convenient to have stereographic projections, and tables of d spacings and interplanar angles for the materials involved. Since not all of these were available or in a complete enough form to be useful, calculations have been compiled for a number of crystal properties of uranium and $\mathrm{U}_{3} \mathrm{Si}$.

Tables I and II 1 ist the d spacings for alpha-uranium at $25^{\circ} \mathrm{C}$ using the lattice constants ${ }^{2} \quad a_{0}=2.8545 \AA, b_{0}=5.8681 \AA$, and $c_{o}=4.9566 \AA$ Table I presents the reflections in hke order while Table II lists them in d spacing order.. All hkes from 001 to 999 were calculated without regard for extinctions. This was done since many forbidden reflections often occur in very thin sections of uranium for a variety of reasons. The calculations were made using the formula for orthorhombic symmetry,

$$
\frac{1}{d^{2}}=\left(\frac{h}{a}\right)^{2}+\left(\frac{k}{b}\right)^{2}+\left(\frac{l}{c}\right)^{2}
$$

may be found in Appendix III of The structure of Metals by Barrett. ${ }^{3}$

Tables III and IV 1 ist the d spacings calculated for the tetragonal cell of $U_{3} S i$ using the lattice constants $a_{0}=6.0290 \AA$ and $c_{0}=8.6963 \AA$, which were calculated from the $\mathrm{kx}$ units given by Zachariasen.4. Again, all hkes were calculated without regard for possible extinctions. The list has been abbreviated, however, since in the tetragonal lattice $h$ and $k$ may be interchanged while the d spacing remains the same. Table III presents the d spacings for $\mathrm{U}_{3} \mathrm{Si}$ in hke order while Table IV lists them in order of dispacing.

The angle, $\phi$, between two crystal plans $\left(h_{1} k_{1} \ell_{1}\right)$ and $\left(h_{2} k_{2} \ell_{2}\right)$ may be determined for orthorhombic, tetragonal and cubic symmetry by using the formula quoted by Barrett ${ }^{3}$ :

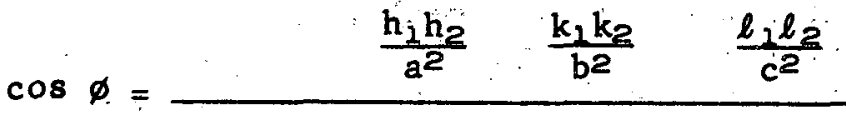

$$
\begin{aligned}
& \sqrt{\left(\frac{h_{j}^{2}}{a^{2}}+\frac{k_{j}^{2}}{b^{2}}+\frac{l_{1}^{2}}{c^{2}}\right)\left(\frac{h_{2}^{2}}{a^{2}}+\frac{k_{2}^{2}}{b^{2}}+\frac{l_{2}^{2}}{c^{2}}\right)}
\end{aligned}
$$


Table I

Interplanar Spacings, dhke, in Angstroms for Alpha-Uranium at $25^{\circ} \mathrm{C}$

Arranged in (hkl) Order

(without regard for extinctions, using $a_{0}=2.8545 \AA$, $b_{0}=5.8681 \AA$, and $\left.c_{0}=4.9566 \AA\right)$

$\begin{array}{lc}\left(\mathrm{hk}_{\ell}\right) & \text { dhkl} \\ & \\ 001 & 4.95660 \\ 002 & 2.47830 \\ 003 & 1.65220 \\ 004 & 1.23915 \\ 0.5 & 0.99132 \\ 006 & 0.82610 \\ 007 & 0.70809 \\ 0.08 & 0.61958 \\ 009 & 0.55073 \\ 010 & 5.86810 \\ 011 & 3.78657 \\ 0112 & 2.28304 \\ 013 & 1.59036 \\ 014 & 1.21241 \\ 015 & 0.97747 \\ 016 & 0.81803 \\ 017 & 0.70299 \\ 018 & 0.61615 \\ 019 & 0.54832 \\ 020 & 2.93405 \\ 021 & 2.52485 \\ 0212 & 1.89329 \\ 023 & 1.43964 \\ 024 & 1.14152\end{array}$

$\begin{array}{ll}025 & 0.93916 \\ 026 & 0.79518 \\ 027 & 0.68832 \\ 028 & 0.60621 \\ 029 & 0.54128 \\ 030 & 1.95603 \\ 031 & 1.81948 \\ 032 & 1.53541 \\ 033 & 1.26219 \\ 034 & 1.04678 \\ 035 & 0.88424 \\ 036 & 0.76101 \\ 037 & 0.66580 \\ 038 & 0.59065 \\ 039 & 0.53012 \\ 040 & 1.46703 \\ 041 & 1.40670 \\ 042 & 1.26243 \\ 043 & 1.09699 \\ 044 & 0.94664 \\ 045 & 0.82137 \\ 046 & 0.71982 \\ 047 & 0.63769 \\ 048 & 0.57076 \\ 049 & 0.51560\end{array}$

$\begin{array}{lc}\text { (hkl) } & \text { dhkl } \\ & \\ 0.50 & 1.17362 \\ 051 & 1.14204 \\ 0.52 & 1.06070 \\ 053 & 0.95680 \\ 054 & 0.85210 \\ 055 & 0.75731 \\ 0.56 & 0.67553 \\ 057 & 0.60628 \\ 058 & 0.54791 \\ 059 & 0.49857 \\ 060 & 0.97802 \\ 061 & 0.95952 \\ 0662 & 0.90974 \\ 063 & 0.84162 \\ 064 & 0.76771 \\ 065 & 0.69622 \\ 066 & 0.63110 \\ 067 & 0.57355 \\ 068 & 0.52339 \\ 069 & 0.47988 \\ 070 & 0.83830 \\ 071 & 0.82656 \\ 072 & 0.79410 \\ 073 & 0.74758 \\ 074 & 0.69434\end{array}$

(hkl) $\quad \underline{d_{h k \ell}}$

$\begin{array}{ll}075 & 0.64011 \\ 076 & 0.58841 \\ 077 & 0.54094 \\ 078 & 0.49826 \\ 079 & 0.46029 \\ 080 & 0.73351 \\ 081 & 0.72561 \\ 082 & 0.70335 \\ 083 & 0.67041 \\ 084 & 0.63121 \\ 085 & 0.58965 \\ 086 & 0.54850 \\ 087 & 0.50944 \\ 088 & 0.47332 \\ 089 & 0.44041 \\ 090 & 0.65201 \\ 091 & 0.64644 \\ 092 & 0.63055 \\ 093 & 0.60649 \\ 094 & 0.57701 \\ 095 & 0.54474 \\ 096 & 0.51180 \\ 097 & 0.47964 \\ 098 & 0.44913 \\ 099 & 0.42073\end{array}$


Table I contd.

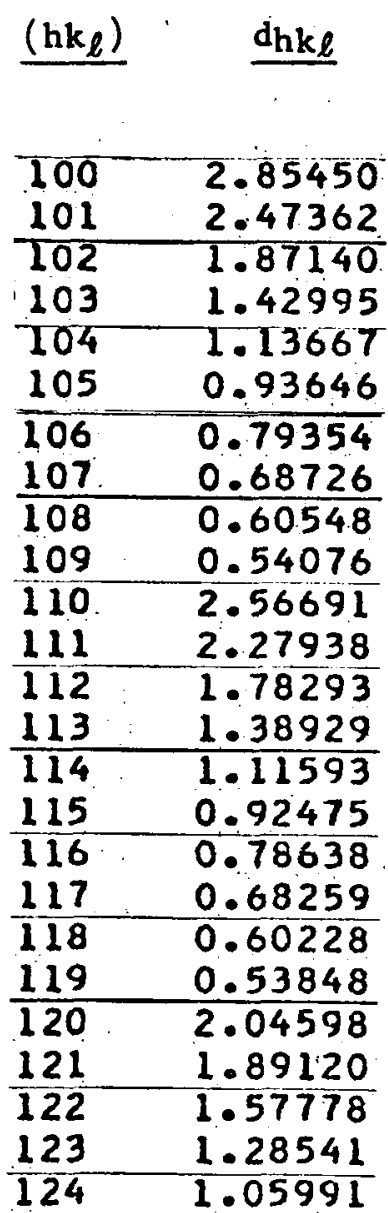

\begin{tabular}{|c|c|}
\hline$\left(\mathrm{hk}_{\boldsymbol{l}}\right)$ & $\mathrm{d}_{\mathrm{hk} \ell}$ \\
\hline 125 & 0.89212 \\
\hline $\begin{array}{l}126 \\
127\end{array}$ & $\begin{array}{l}0.76602 \\
0.66915\end{array}$ \\
\hline 128 & 0.59298 \\
\hline 129 & 0.53180 \\
\hline 130 & 1.61355 \\
\hline 131 & 3430 \\
\hline 132 & 1.35221 \\
\hline 133 & 1.15437 \\
\hline 134 & $\begin{array}{l}0.98278 \\
0.84465\end{array}$ \\
\hline $\begin{array}{l}135 \\
136\end{array}$ & $\frac{0.84465}{0.73533}$ \\
\hline 137 & 0.64840 \\
\hline 138 & 0.57840 \\
\hline 139 & 0.52121 \\
\hline 140 & 1.30479 \\
\hline 141 & 1.26181 \\
\hline 142 & 1.15455 \\
\hline 143 & 1.02398 \\
\hline 144 & 0.89852 \\
\hline 145 & 8935 \\
\hline 146 & 0.69797 \\
\hline 147 & 0.62235 \\
\hline 148 & 0.55968 \\
\hline 149 & 0.5073 \\
\hline
\end{tabular}

\begin{tabular}{lc}
$\left(\mathrm{hk}_{\ell}\right)$ & dhk \\
\hline & \\
150 & 1.08546 \\
151 & 1.06033 \\
\hline 152 & 0.99427 \\
153 & 0.90719 \\
\hline 154 & 0.81650 \\
155 & 0.73199 \\
\hline 156 & 0.65737 \\
157 & 0.59306 \\
\hline 158 & 0.53809 \\
159 & 0.49113 \\
\hline 160 & 0.92522 \\
161 & 0.90951 \\
\hline 162 & 0.86678 \\
163 & 0.80726 \\
\hline 164 & 0.74136 \\
165 & 0.67639 \\
\hline 166 & 0.61621 \\
167 & 0.56231 \\
\hline 168 & 0.51481 \\
169 & 0.47324 \\
\hline 170 & 0.80433 \\
171 & 0.79395 \\
\hline 172 & 0.76505 \\
173 & 0.72319 \\
\hline 174 & 0.67466
\end{tabular}

$\underline{\left(h_{\ell}\right)} \quad \underline{d_{k} \ell}$

\begin{tabular}{ll}
175 & 0.62460 \\
176 & 0.57629 \\
177 & 0.53148 \\
178 & 0.49084 \\
179 & 0.45442 \\
\hline 180 & 0.71043 \\
181 & 0.70324 \\
182 & 0.68293 \\
183 & 0.65265 \\
184 & 0.61632 \\
185 & 0.57746 \\
\hline 186 & 0.53864 \\
187 & 0.50152 \\
\hline 188 & 0.46695 \\
189 & 0.43526 \\
190 & 0.63564 \\
191 & 0.63048 \\
192 & 0.61571 \\
193 & 0.59325 \\
194 & 0.56557 \\
195 & 0.53509 \\
196 & 0.50377 \\
197 & 0.47301 \\
\hline 198 & 0.44368 \\
199 & 0.41623
\end{tabular}


Table I contd.

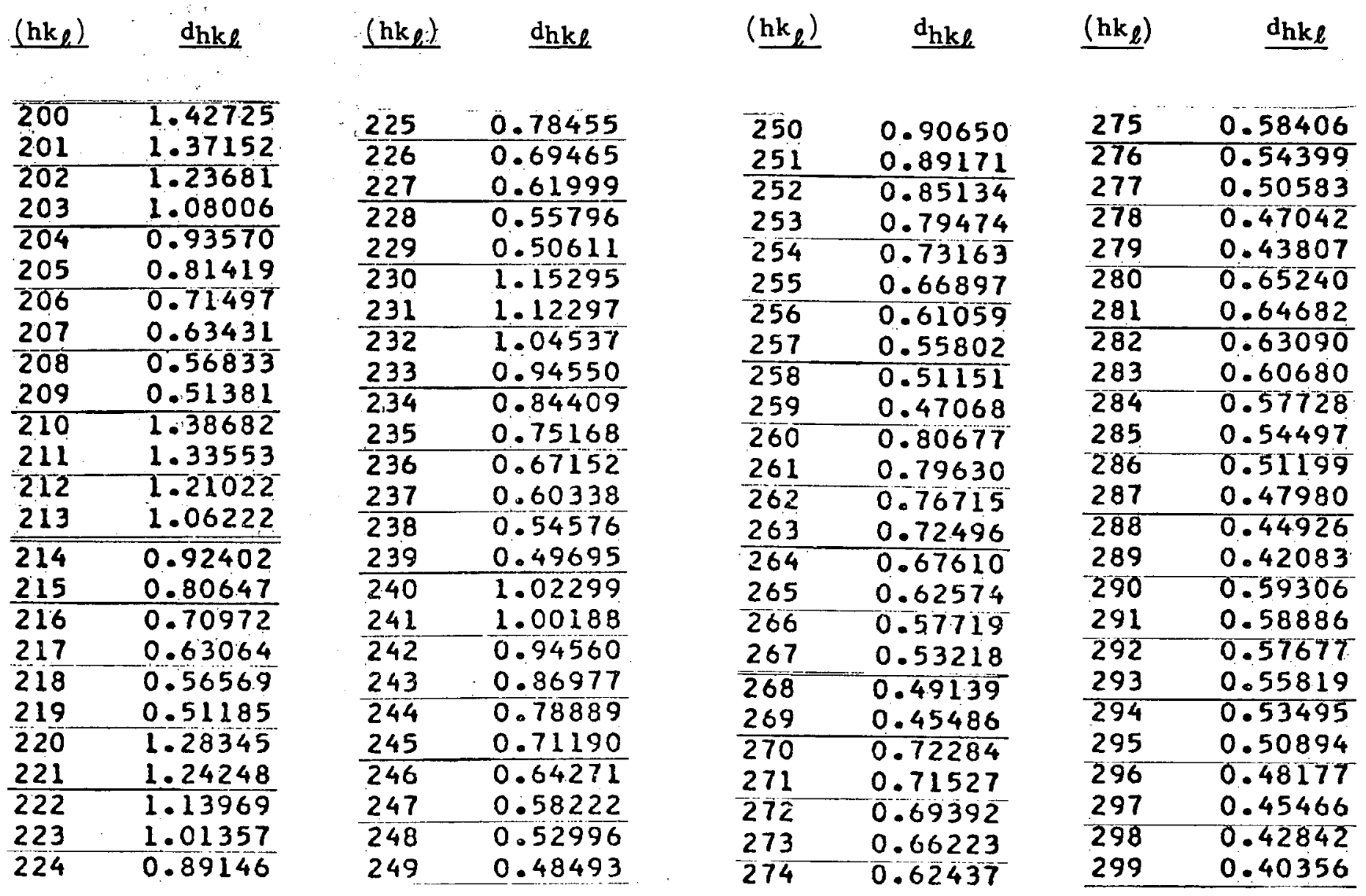


Table I contd.

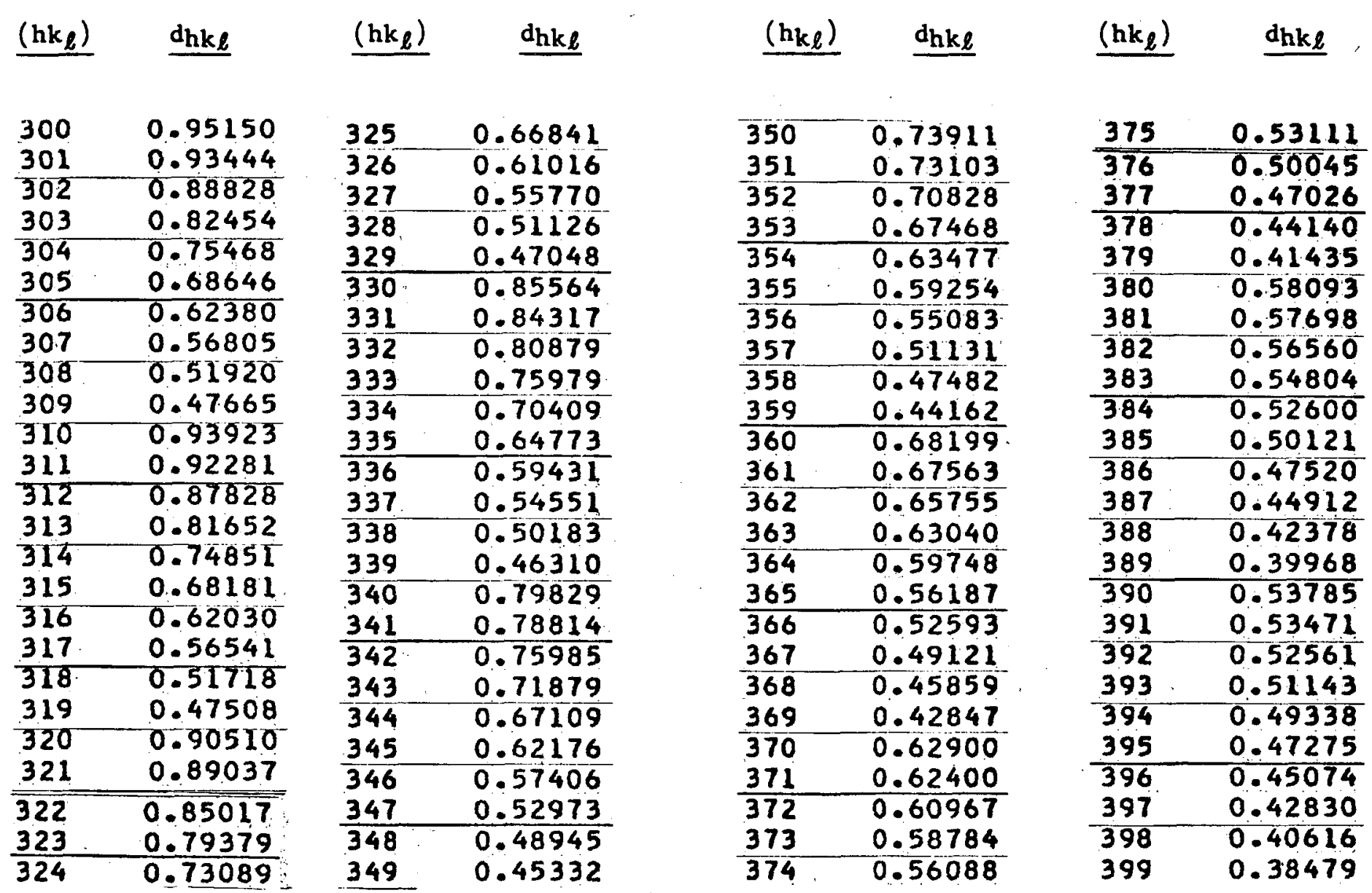


Table I contd.

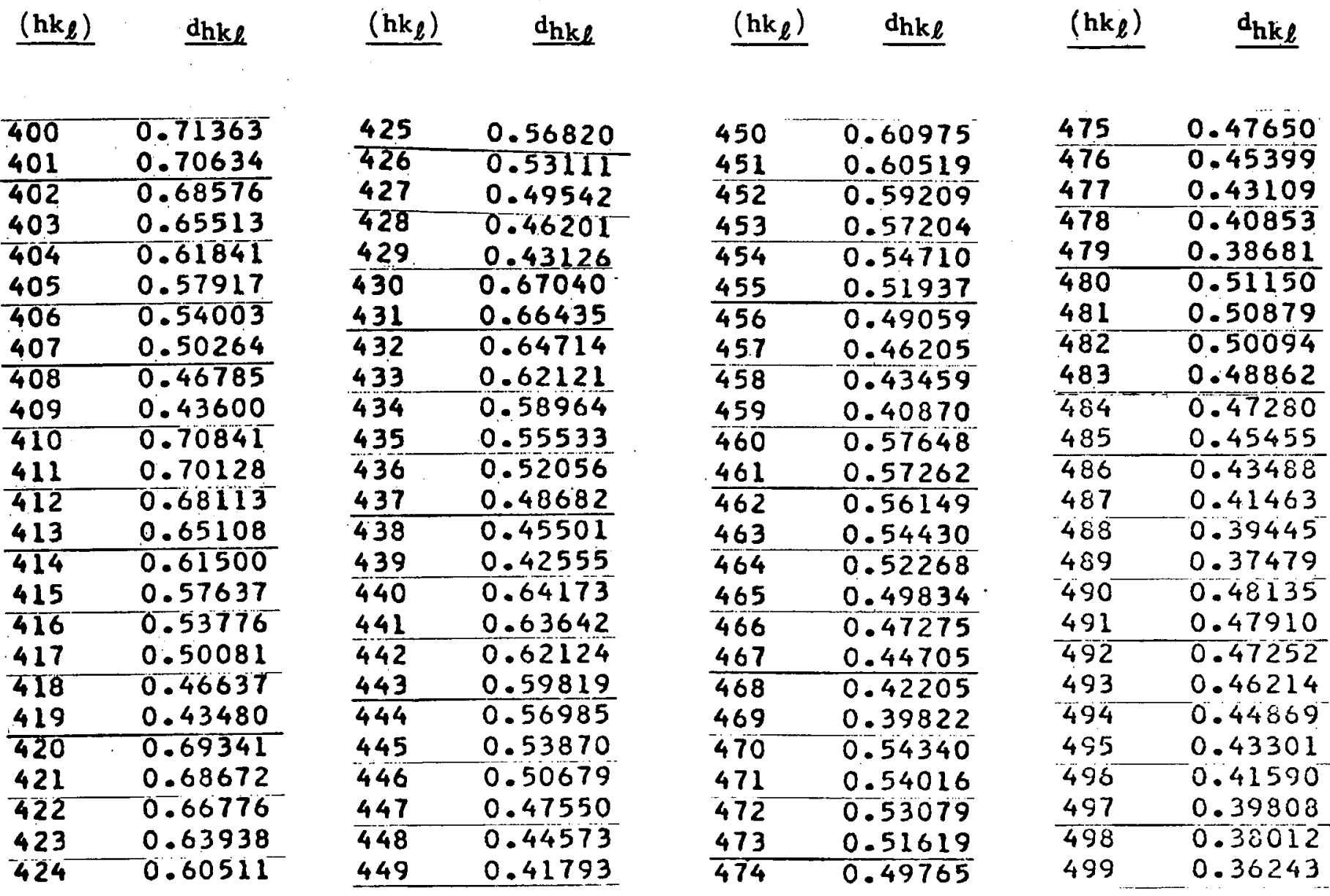


Table I contd.

\begin{tabular}{|c|c|}
\hline$\left(\mathrm{hk}_{\ell}\right)$ & dhk $\ell$ \\
\hline $\begin{array}{l}500 \\
501 \\
502 \\
503\end{array}$ & $\begin{array}{l}0.57090 \\
0.56715 \\
0.55633 \\
0.53959\end{array}$ \\
\hline 504 & 0.51852 \\
\hline 505 & 0.49472 \\
\hline 506 & 0.46966 \\
\hline 507 & 0.44444 \\
\hline 508 & 0.41984 \\
\hline 509 & 0.39637 \\
\hline 510 & 0.56822 \\
\hline 511 & 0.5645 \\
\hline 512 & 0.5538 \\
\hline 513 & 0.53733 \\
\hline 514 & 0.51650 \\
\hline 515 & 0.49298 \\
\hline 516 & 0.46816 \\
\hline 517 & 0.44317 \\
\hline 518 & 0.41877 \\
\hline 519 & 0.3954 \\
\hline 520 & $Q .56039$ \\
\hline 521 & 0.55684 \\
\hline 522 & 0.54659 \\
\hline 523 & 0.53069 \\
\hline 524 & 0.51060 \\
\hline
\end{tabular}

\begin{tabular}{|c|c|}
\hline$\left(\mathrm{hk}_{\ell}\right.$ & dhk \\
\hline 525 & 0.48784 \\
\hline 526 & 0.46376 \\
\hline 527 & 0.43943 \\
\hline 523 & 0.41561 \\
\hline 529 & 0.39280 \\
\hline 530 & 0.54003 \\
\hline 531 & 0.54472 \\
\hline 532 & 0.53511 \\
\hline 533 & 0.52017 \\
\hline 534 & 0.50120 \\
\hline 535 & 0.47952 \\
\hline-536 & 0.45668 \\
\hline 537 & 0.43339 \\
\hline 538 & 0.41049 \\
\hline 539 & 0.38847 \\
\hline 540 & 0.53203 \\
\hline 541 & 2899 \\
\hline 542 & 2018 \\
\hline 543 & 642 \\
\hline 544 & 888 \\
\hline 545 & 0.2 \\
\hline 546 & 0.44730 \\
\hline 547 & 253 \\
\hline 54 & 10364 \\
\hline 549 & 0.38264 \\
\hline
\end{tabular}

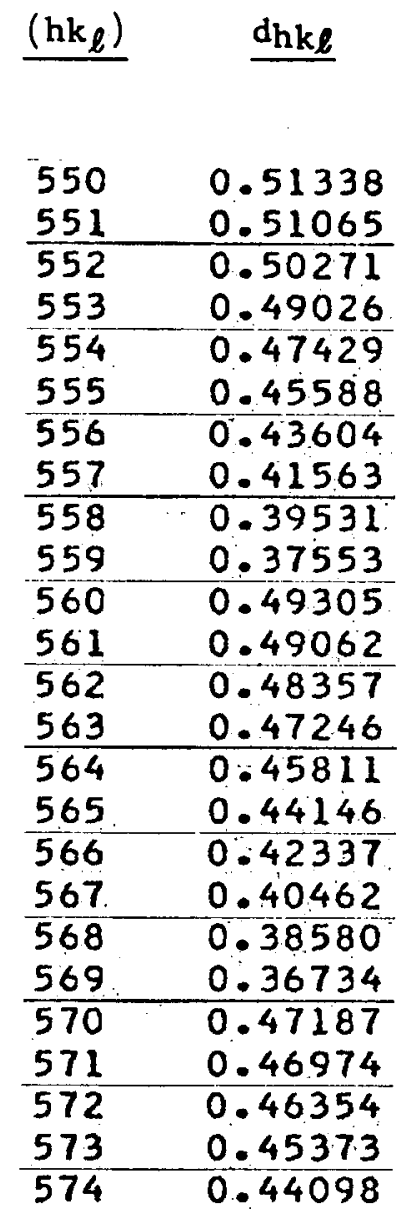

$\underline{\left(\text { hk }_{\ell}\right)} \underline{\mathrm{dhk}_{\ell}}$

\begin{tabular}{ll}
575 & 0.42606 \\
\hline 576 & 0.40974 \\
577 & 0.39267 \\
578 & 0.37539 \\
579 & 0.35833 \\
\hline 580 & 0.45052 \\
581 & 0.44868 \\
\hline 582 & 0.44326 \\
583 & 0.43466 \\
584 & 0.42341 \\
585 & 0.41015 \\
586 & 0.39553 \\
587 & 0.38011 \\
\hline 588 & 0.36438 \\
589 & 0.34871 \\
\hline 590 & 0.42952 \\
591 & 0.42791 \\
\hline 592 & 0.42321 \\
593 & 0.41570 \\
\hline 594 & 0.40583 \\
595 & 0.39411 \\
\hline 596 & 0.38109 \\
597 & 0.36724 \\
\hline 598 & 0.35299 \\
599 & 0.33869 \\
\hline
\end{tabular}


Table I contd.

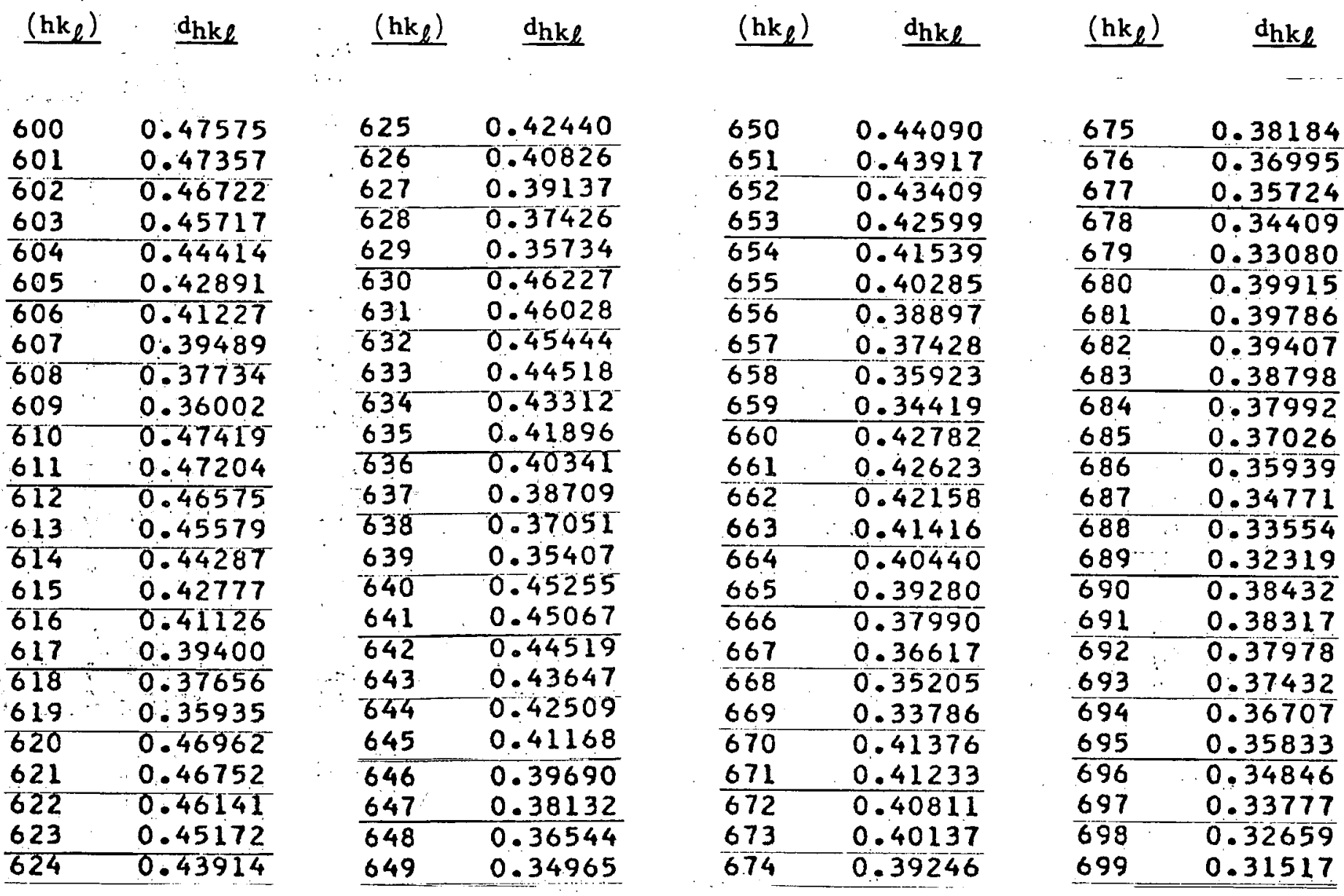


Table I contd.

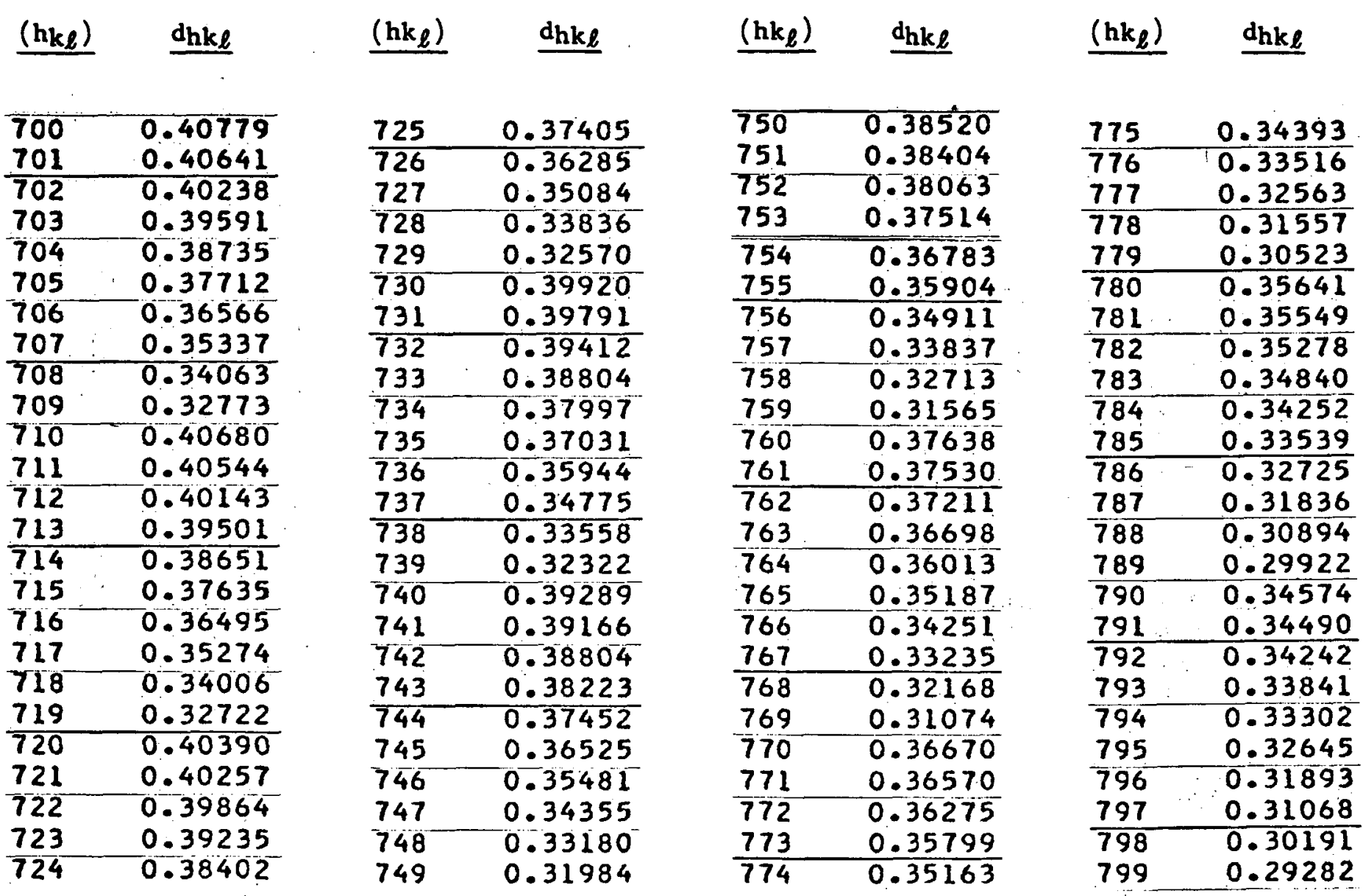


Table I contd.

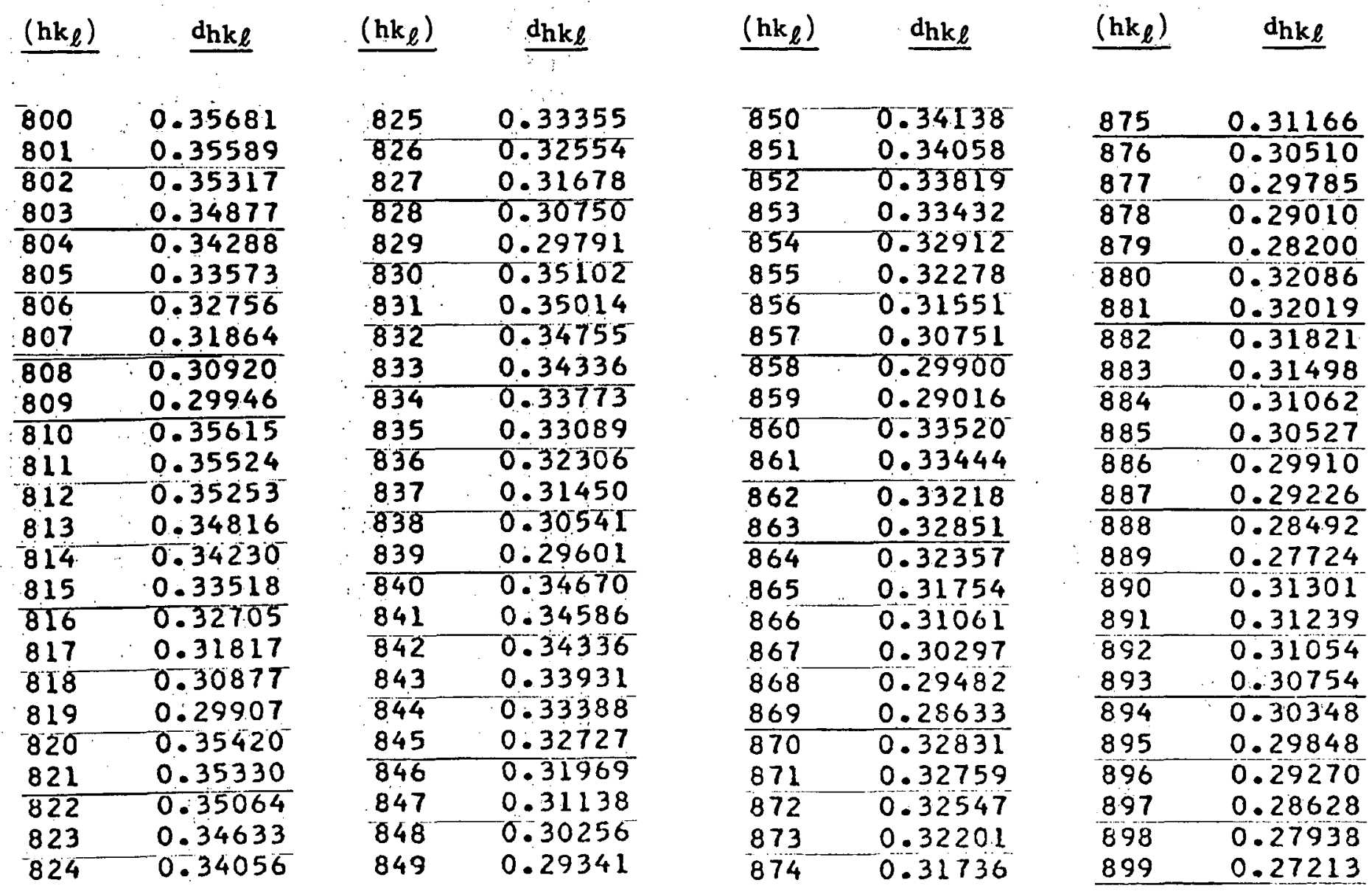


Table I contd.

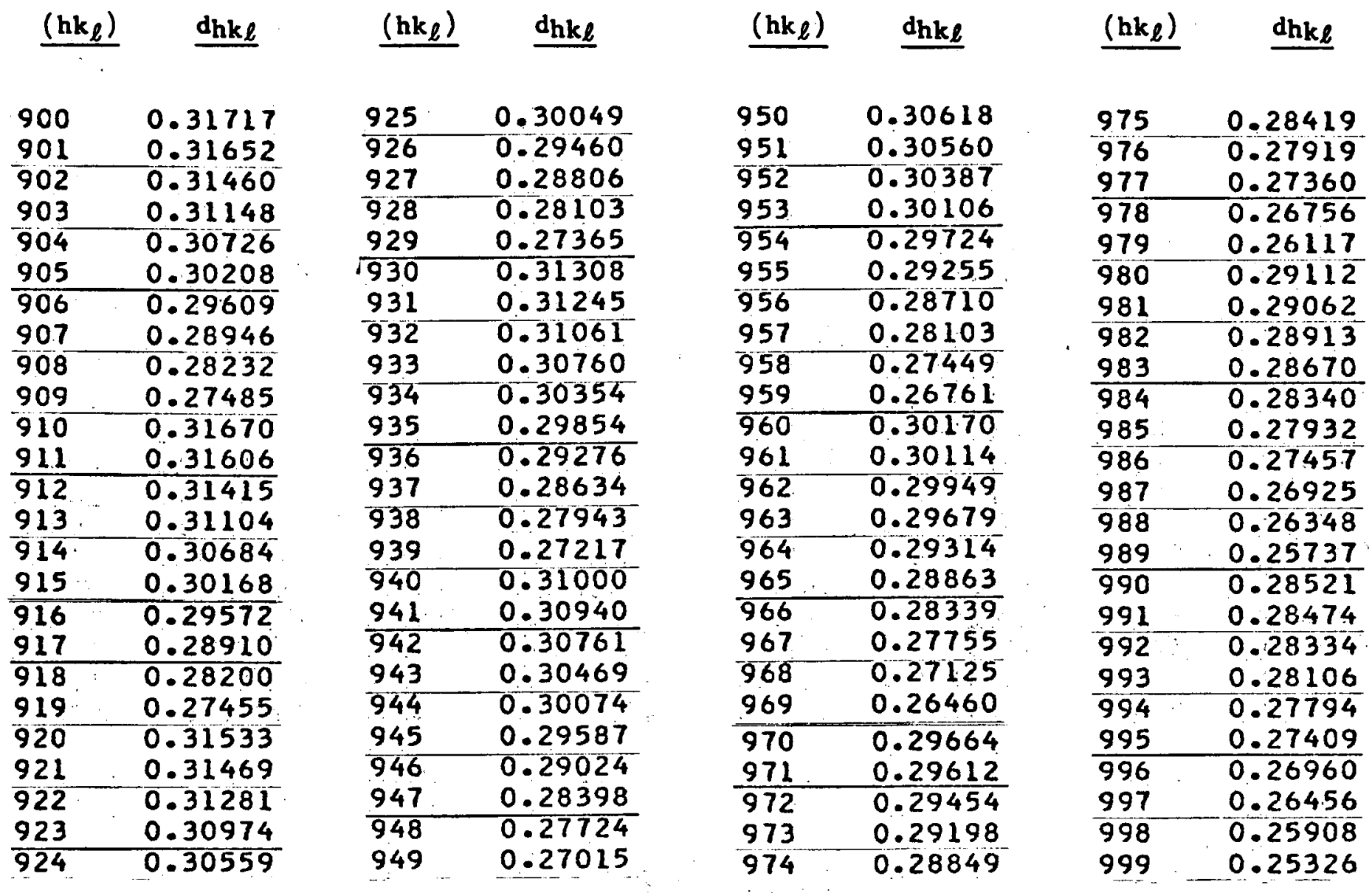


Table II

Interplanar Spacing, $\mathrm{d}_{\mathrm{h} \ell}$, , in Angstroms for Alpha-Uranium at $25^{\circ} \mathrm{C}$

Arranged in dhke Order

(without regard for extinctions, using $a_{0}=2.8545 \AA, b_{0}=5.8681 \AA, c_{0}=4.9566 \AA$ )

\begin{tabular}{|c|c|c|c|c|c|c|c|}
\hline (hkl) & dhkl & (hke) & dhk & (hke) & dhkl & (hkl) & $\mathrm{d}_{\mathrm{hk} \ell}$ \\
\hline 010 & 5.86810 & 132 & 1.35221 & 143 & 1.02398 & 224 & 0.89146 \\
\hline 001 & 4.95660 & 211 & 1.33553 & 240 & 1.02299 & 321 & 0.89037 \\
\hline 011 & 3.78657 & 140 & 1.30479 & 223 & 1.01357 & 302 & 0.88828 \\
\hline 020 & 2.93405 & 123 & 1.28541 & 241 & 1.00188 & 035 & 0.88424 \\
\hline 100 & 2.85450 & 220 & 1.28345 & 152 & 0.99427 & 312 & 0.87828 \\
\hline 110 & 2.56691 & 042 & 1.26243 & 005 & 0.99132 & 243 & 0.86977 \\
\hline$\infty 1$ & 2.52485 & 033 & 1.26219 & 134 & 0.98278 & 162 & 0.86678 \\
\hline 002 & 2. 47830 & 141 & 1.26181 & 060 & 0.97802 & 330 & 0.85564 \\
\hline 101 & 2.47362 & 221 & 1.24248 & 015 & 0.97747 & 054 & 0.85210 \\
\hline 012 & 2.28304 & 004 & 1.23915 & 061 & 0.95952 & 252 & 0.85134 \\
\hline 111 & 2.27938 & 202 & 1.23681 & 053 & 0.95680 & 322 & 0.85017 \\
\hline 120 & 2.04598 & 014 & 1.21241 & 300 & 0.95150 & 135 & 0.84465 \\
\hline 030 & 1.95603 & 212 & 1.21022 & 044 & 0.94664 & 234 & 0.84409 \\
\hline ஹ2 & i. 89329 & 050 & 1.17362 & 242 & 0.94560 & 331 & 0.84317 \\
\hline 121 & 1.89120 & 142 & 1.15455 & 233 & 0.94550 & 063 & 0.84162 \\
\hline 102 & 1.87140 & 133 & 1.15437 & 310 & 0.93923 & 070 & 0.83830 \\
\hline 031 & 1.81948 & 230 & 1.15295 & 025 & 0.93916 & 071 & 0.82656 \\
\hline 112 & 1.78293 & 051 & 1.14204 & 105 & 0.93646 & 006 & 0.82610 \\
\hline 003 & 1.65220 & 024 & 1.14152 & 204 & 0.93570 & 303 & 0.82454 \\
\hline 130 & 1.61355 & 222 & 1.13969 & 301 & 0.93444 & 045 & 0.82137 \\
\hline 013 & 1.59036 & 104 & 1.13667 & 160 & 0.92522 & 016 & 0.81803 \\
\hline 122 & 1.57778 & 231 & 1.12297 & 11.5 & 0.92475 & 313 & 0.81652 \\
\hline 032 & 1.53541 & 114 & 1.11593 & 214 & 0.92402 & 154 & 0.81650 \\
\hline 131 & 1.53430 & 043 & 1.09699 & 311 & 0.92281 & 205 & 0.81419 \\
\hline 040 & 1.46703 & 150 & 1.08546 & 062 & 0.90974 & 332 & 0.80879 \\
\hline 023 & 1.43964 & 203 & 1.08006 & 161 & 0.90951 & 163 & 0.80726 \\
\hline 103 & 1.42995 & 213 & 1.06222 & 153 & 0.90719 & 260 & 0.80677 \\
\hline 200 & 1.42725 & 052 & 1.06070 & 250 & 0.90650 & 215 & 0.80647 \\
\hline 041 & 1.40670 & 151 & 1.06033 & 320 & 0.90510 & 170 & 0.80433 \\
\hline 113 & 1.38929 & 124 & 1.05991 & 144 & 0.89852 & 340 & 0.79829 \\
\hline 210 & 1.38682 & 034 & 1.04678 & 125 & 0.89212 & 261 & 0.79630 \\
\hline 201 & 1.37152 & 232 & 1.04537 & 251 & 0.89171 & 026 & 0.79518 \\
\hline
\end{tabular}


Table II contd.

\begin{tabular}{|c|c|c|c|c|c|c|c|}
\hline (hke) & dikk & (hke) & $\mathrm{d}_{\mathrm{hk} \boldsymbol{\ell}}$ & (hke) & $\mathrm{d}_{\mathrm{hk \ell}}$ & (hke) & dike \\
\hline 253 & 0.79474 & 173 & 0.72319 & 360 & 0.68199 & 246 & 0.64271 \\
\hline 072 & 0.79410 & 270 & 0.72284 & 315 & 0.68181 & 440 & 0.64173 \\
\hline 171 & 0.79395 & 046 & 0.71982 & 412 & 0.68113 & 075 & 0.64011 \\
\hline 323 & 0.79379 & 343 & 0.71879 & 165 & 0.67639 & 423 & 0.63938 \\
\hline 106 & 0.79354 & 271 & 0.71527 & 264 & 0.67610 & 047 & 0.63769 \\
\hline 145 & 0.78935 & 206 & 0.71497 & 361 & 0.67563 & 441 & 0.63642 \\
\hline 244 & 0.78889 & 400 & 0.71363 & 056 & 0.67553 & 190 & 0.63564 \\
\hline 341 & 0.78814 & 245 & 0.71190 & 353 & 0.67468 & 354 & 0.63477 \\
\hline 116 & 0.78638 & 180 & 0.71043 & 174 & 0.67466 & 207 & 0.63431 \\
\hline 225 & 0.78455 & 216 & 0.70972 & 236 & 0.67152 & 084 & 0.63121 \\
\hline 064 & 0.76771 & 410 & 0.70841 & 344 & 0.67109 & 066 & 0.63110 \\
\hline 262 & 0.76715 & 352 & 0.7 .0828 & 083 & 0.67041 & 282 & 0.63090 \\
\hline 126 & 0.76602 & 007 & 0.70809 & 430 & 0.67040 & 217 & 0.63064 \\
\hline 172 & 0.76505 & 401 & 0.70634 & 127 & 0.66915 & 092 & 0.63055 \\
\hline 036 & 0.76101 & 334 & 0.70409 & 255 & 0.66897 & 191 & 0.63048 \\
\hline 342 & 0.75985 & 082 & 0.70335 & 325 & 0.66841 & 363 & 0.63040 \\
\hline 333 & 0.75979 & 181 & 0.70324 & 422 & 0.66776 & 370 & 0.62900 \\
\hline 055 & 0.75731 & 017 & 0.70299 & 037 & 0.66580 & 265 & 0.62574 \\
\hline 304 & 0.75468 & 411 & 0.70128 & 431 & $0: 66435$ & 175 & 0.62460 \\
\hline 235 & 0.75168 & 146 & 0.69797 & 273 & 0.66223 & 274 & 0.62437 \\
\hline 314 & 0.74851 & 065 & 0.69622 & 362 & 0.65755 & 371 & 0.62400 \\
\hline 073 & 0.74758 & 226 & 0.69465 & 156 & 0.65737 & 306 & 0.62380 \\
\hline 164 & 0.74136 & 074 & 0.69434 & 403 & 0.65513 & 147 & 0.62235 \\
\hline 350 & 0.73911 & 272 & 0.69392 & 183 & 0.65265 & 345 & 0.62176 \\
\hline 136 & 0.73533 & 420 & 0.69341 & 280 & 0.65240 & 442 & 0.62124 \\
\hline 080 & 0.73351 & 027 & 0.68832 & 090 & 0.65201 & 433 & 0.62121 \\
\hline 155 & 0.73199 & 107 & 0.68726 & 413 & 0.65108 & 316 & 0.62030 \\
\hline 254 & 0.73163 & 421 & 0.68672 & 137. & 0.64840 & 227 & 0.61999 \\
\hline 351 & 0.73103 & 305 & 0.68646 & 335 & 0.64773 & 008 & 0.61958 \\
\hline 324 & 0.73089 & 402 & 0.68576 & 432 & 0.64714 & 404 & 0.61841 \\
\hline 081 & 0.72561 & 182 & 0.68293 & 281 & 0.64682 & 184 & 0.61632 \\
\hline 263 & 0.72496 & 117 & 0.68259 & 091 & 0.64644 & 166 & 0.61621 \\
\hline
\end{tabular}


Table II contd.

\begin{tabular}{|c|c|c|c|c|c|c|c|}
\hline (hkl) & $\mathrm{d}_{\mathrm{k}}^{\mathrm{k}} \mathrm{k} \ell$ & (hke) & $d_{h k \ell}$ & (hke) & $\mathrm{d}_{\mathbf{h} \mathbf{k} \ell}$ & (hke) & $\mathrm{dhk}_{\mathrm{\ell}}$ \\
\hline 018 & 0.61615 & 247 & 0.58222 & 462 & 0.56149 & 471 & 0.54016 \\
\hline 192 & 0.61571 & 380 & 0.58093 & 374 & 0.56088 & 406 & 0.54003 \\
\hline 414 & 0.61500 & 405 & $0: 57971$ & 520 & 0.56039 & 503 & 0.53959 \\
\hline 256 & 0.61059 & 138 & 0.57840 & 148 & 0.55968 & 445 & 0.53870 \\
\hline 326 & 0.61016 & 185 & 0.57746 & 293 & 0.55819 & 186 & 0.53864 \\
\hline 450 & 0.60975 & 284 & 0.57728 & 257 & 0.55802 & 119 & 0.53848 \\
\hline 372 & 0.60967 & 266 & 0.57719 & 228 & 0.55796 & 158 & 0.53809 \\
\hline 283 & 0.60680 & 094 & 0.57701 & 327 & 0.55770 & 390 & 0.53785 \\
\hline 093 & 0.60649 & 381 & 0.57698 & 521 & 0.55684 & 416 & 0.53776 \\
\hline 057 & 0.60628 & 292 & 0.57677 & 502 & 0.55633 & 513 & 0.53733 \\
\hline 028 & 0.60621 & 460 & 0.57648 & 435 & 0.55533 & 532 & 0.53511 \\
\hline 108 & 0.60548 & 415 & 0.57637 & 512 & 0.55385 & 195 & 0.53509 \\
\hline 451 & 0.60519 & 176 & 0.57629 & 356 & 0.55083 & 294 & 0.53495 \\
\hline 424 & 0.60511 & 346 & 0.57406 & 009 & 0.55073 & 391 & 0.53471 \\
\hline 237 & 0.60338 & 067 & 0.57355 & 086 & 0.54850 & 267 & 0.53218 \\
\hline 118 & 0.60228 & 461 & 0.57262 & 019 & 0.54832 & 540 & 0.53203 \\
\hline 443 & 0.59819 & 453 & 0.57204 & 383 & 0.54804 & 129 & 0.53180 \\
\hline 364 & 0.59748 & 500 & 0.57090 & 530 & 0.54803 & 177 & 0.53148 \\
\hline 336 & 0.59431 & 048 & 0.57076 & 058 & 0.54791 & 426 & 0.53111 \\
\hline 193 & 0.59325 & 444 & 0.56985 & 454 & 0.54710 & 375 & 0.53111 \\
\hline 290 & 0.59306 & 208 & 0.56833 & 522 & 0.54659 & 472 & 0.53079 \\
\hline 157 & 0.59306 & 510 & 0.56822 & 238 & 0.54576 & 523 & 0.53069 \\
\hline-128 & 0.59298 & 425 & 0.56820 & 337 & 0.54551 & 039 & 0.53012 \\
\hline 355 & 0.59254 & 307 & $0: 56805$ & 285 & 0.54497 & 248 & 0.52996 \\
\hline 452 & 0.59209 & 501 & 0.56715 & 095 & 0.54474 & 347 & 0.52973 \\
\hline 038 & $0: 59065$ & 218 & 0.56569 & 531 & 0.54472 & 541 & 0.52899 \\
\hline 085 & 0.58965 & 382 & 0.56560 & 463 & 0.54430 & 384 & 0.52600 \\
\hline 434 & 0.58964 & 194 & 0.56557 & 276 & 0.54399 & 366 & 0.52593 \\
\hline 291 & 0.58886 & 317 & 0.56541 & 470 & 0.54340 & 392 & 0.52561 \\
\hline 076 & 0.58841 & 511 & 0.56452 & 029 & 0.54128 & 068 & 0.52339 \\
\hline 373 & 0.58784 & 167 & 0.56231 & 077 & 0.54094 & 464 & 0.52268 \\
\hline 275 & 0.58406 & 365 & 0.56187 & 109 & 0.54076 & 139. & 0.52121 \\
\hline
\end{tabular}


Table II contd.

\begin{tabular}{|c|c|c|c|c|c|c|c|}
\hline (hke) & dhke & (hke) & dhke & (hke) & dhk $\ell^{\prime}$ & (hkl) & dhke \\
\hline 436 & 0.52056 & 552 & 0.50271 & 562 & 0.48357 & 571 & $0.4697^{4}$ \\
\hline 542 & 0.52018 & 407 & 0.50264 & 296 & 0.48177 & 506 & 0.46966 \\
\hline 533 & 0.52017 & 338 & 0.50183 & 490 & 0.48135 & 620 & 0.46962 \\
\hline 455 & 0.51937 & 187 & 0.50152 & 069 & 0.47988 & 545 & 0.46879 \\
\hline 308 & 0.51920 & 385 & 0.50121 & 287 & 0.47980 & 516 & 0.46816 \\
\hline 504 & 0.51852 & 534 & 0.50120 & 097 & 0.47964 & 408 & 0.46785 \\
\hline 318 & 0.51718 & 482 & 0.50094 & 535 & 0.47962 & 621 & 0.46752 \\
\hline 514 & 0.51650 & 417 & 0.50081 & 491 & 0.47910 & 602 & 0.46722 \\
\hline 473 & 0.51619 & 376 & 0.50045 & 309 & 0.47665 & 188 & 0.46695 \\
\hline 049 & 0.51560 & 059 & 0.49857 & 475 & 0.47650 & 418 & 0.46637 \\
\hline 168 & 0.51481 & 465 & 0.49834 & 600 & 0.47575 & 612 & 0.46575 \\
\hline 209 & 0.51381 & 078 & 0.49826 & 447 & 0.47550 & 526 & 0.46376 \\
\hline 550 & 0.51338 & 474 & 0.49765 & 386 & 0.47520 & 572 & 0.46354 \\
\hline 286 & 0.51199 & 239 & 0.49695 & 319 & 0.47508 & 339 & 0.46310 \\
\hline 219 & 0.51185 & 427 & 0.49542 & 358 & 0.47482 & 630 & 0.46227 \\
\hline 096 & 0.51180 & 505 & 0.49472 & 554 & 0.47429 & 493 & 0.46214 \\
\hline 258 & 0.51151 & 394 & 0.49338 & 610 & 0.47419 & 457 & 0.46205 \\
\hline 480 & 0.51150 & 560 & 0.49305 & 601 & 0.47357 & 428 & 0.46201 \\
\hline 393 & 0.51143 & 515 & 0.49298 & 088 & 0.47332 & 622 & 0.461 .41 \\
\hline 357 & 0.51131 & 268 & 0.49139 & 169 & 0.47324 & 079 & 0.46029 \\
\hline 328 & 0.51126 & 367 & 0.49121 & 197 & 0.47301 & 631 & 0.46028 \\
\hline 551 & 0.51065 & 159 & 0.49113 & 484 & 0.47280 & 368 & 0.45859 \\
\hline 524 & 0.51060 & 178 & 0.49084 & 466 & 0.47275 & 564 & 0.45811 \\
\hline 087 & 0.50944 & 561 & 0.49062 & 395 & 0.47275 & 603 & 0.45717 \\
\hline 295 & 0.50894 & 456 & 0.49059 & 492 & 0.47252 & 536. & 0.45668 \\
\hline 481 & 0.50879 & 553 & 0.49026 & 503 & 0.47246 & 555 & 0.45588 \\
\hline 149 & 0.50739 & 348 & 0.48945 & 611 & 0.47204 & 613 & 0.45579 \\
\hline 446 & 0.50679 & & 0.48888 & 570 & 0.47187 & & 0.45501 \\
\hline 543 & 0.50642 & 483 & 0.48862 & 259 & 0.47068 & 269 & 0.45486 \\
\hline 229 & 0.50611 & 525 & 0.48784 & 329 & 0.47048 & 297 & 0.45466 \\
\hline 277 & 0.50583 & 437 & 0.48682 & 278 & 0.47042 & 485 & 0.45455 \\
\hline 196 & 0.50377 & 249 & 0.48493 & 377 & 0.47026 & 632 & 0.45444 \\
\hline
\end{tabular}


Table II contd.

\begin{tabular}{|c|c|c|c|c|c|c|c|}
\hline$(\mathrm{hk} b)$ & $\therefore \mathrm{d}_{\mathrm{hk} \ell}$ & (hkl) & dhk $\ell$ & (hke) & $\mathrm{d}_{\mathrm{h} k \ell} \boldsymbol{l}$ & (hke) & dhk $\ell$ \\
\hline 179 & 0.45442 & 651 & 0.43917 & 388 & 0.42378 & 672 & 0.40811 \\
\hline 476 & 0.45399 & 624 & 0.43914 & 584 & 0.42341 & 700 & 0.40779 \\
\hline 573 & 0.45373 & 279 & 0.43807 & 566 & 0.42337 & 710 & 0.40680 \\
\hline 349 & 0.45332 & 643 & 0.43647 & 592 & 0.42321 & 701 & 0.40641 \\
\hline 640 & 0.45255 & 556 & 0.43604 & 468 & 0.42205 & 398 & 0.40616 \\
\hline 623 & 0.45172 & 409 & 0.43600 & 662 & 0.42158 & 594 & 0.40583 \\
\hline 396 & 0.45074 & 189 & 0.43526 & 289 & 0.42083 & 711 & 0.40544 \\
\hline 641 & 0.45067 & 486 & 0.43488 & 099 & 0.42073 & 567 & 0.40462 \\
\hline 580 & 0.45052 & 419 & 0.43480 & 508 & 0.41984 & 664 & 0.40440 \\
\hline 288 & 0.44926 & 583 & 0.43466 & 635 & 0.41896 & 720 & 0.40390 \\
\hline 098 & 0.44913 & 458 & 0.43459 & 518 & 0.41877 & 548 & 0.40364 \\
\hline 387 & 0.44912 & 652 & 0.43409 & 449 & 0.41793 & 299 & 0.40356 \\
\hline 494 & 0.44869 & 537 & 0.43339 & 199 & 0.41623 & 636 & 0.40341 \\
\hline 581 & 0.44868 & 634 & 0.43312 & 496 & 0.41590 & 655 & 0.40285 \\
\hline 546 & 0.44730 & 495 & 0.43301 & 593 & 0.41570 & 721 & 0.40257 \\
\hline 467 & 0.44705 & 429 & 0.43126 & 557 & 0.41563 & 7.02 & 0.40238 \\
\hline 448 & 0.44573 & 477 & 0.43109 & 528 & 0.41561 & 712 & 0.40143 \\
\hline 642 & 0.44519 & 590 & 0.42952 & 654 & 0.41539 & 673 & 0.40137 \\
\hline 633 & 0.44518 & 605 & 0.42891 & 487 & 0.41463 & 389 & 0.39968 \\
\hline 507 & 0.44444 & 369 & 0.42847 & 379 & 0.41435 & 730 & 0.39920 \\
\hline 604 & 0.44414 & 298 & 0.42842 & 663 & 0.41416 & 680 & 0.39915 \\
\hline 198 & 0.44368 & 39.7 & 0.52830 & 670 & 0.41376 & 722 & 0.39864 \\
\hline 582 & 0.44326 & 591 & 0.42791 & 671 & 0.41233 & 469 & 0.39822 \\
\hline 517. & 0.44317 & 660 & 0.42782 & 606 & 0.41227 & 497 & 0.39808 \\
\hline 614 & 0.44287 & 615 & 0.42777 & 645 & 0.41168 & 731 & 0.39791 \\
\hline 359 & 0.44162 & 661 & 0.42623 & 616 & 0.41126 & 681 & 0.39786 \\
\hline 565 & 0.44146 & 575 & 0.42606 & 538 & 0.41049 & 646 & 0.39690 \\
\hline 378 & 0.44140 & 653 & 0.42599 & 585 & 0.41015 & 509 & 0.39637 \\
\hline 574 & 0.44098 & 439 & 0.42555 & 576 & 0.40974 & 703 & 0.39591 \\
\hline 650 & 0.44090 & 547 & 0.42535 & 459 & 0.40870 & 586 & 0.39553 \\
\hline 089 & 0.44041 & 644 & 0.42509 & 478 & 0.40853 & 519 & 0.39546 \\
\hline 527 & 0.43943 & 625 & 0.42440 & 626 & 0.40826 & 558 & 0.39531 \\
\hline
\end{tabular}


Table II contd.

\begin{tabular}{|c|c|c|c|c|c|c|c|}
\hline (hke) & dhke & (hke) & $\underline{\mathrm{dhk}_{\boldsymbol{L}}}$ & (kke) & dhke & $(h k \ell)$ & $d_{\text {hk }}$ \\
\hline 713 & 0.39501 & 743 & 0.38223 & 569 & 0.36734 & 811 & 0.35524 \\
\hline 607 & 0.39489 & 675 & 0.38184 & 597 & 0.36724 & 746 & 0.35481 \\
\hline 488 & 0.39445 & 647 & 0.38132 & 694 & 0.36707 & 820 & 0.35420 \\
\hline 732 & 0.39412 & 596 & 0.38109 & 763 & 0.36698 & 639 & 0.35407 \\
\hline 595 & 0.39411 & 752 & 0.38063 & 770 & 0.36670 & 707 & 0.35337 \\
\hline 682 & 0.39407 & 498 & 0.38012 & 667 & 0.36617 & 821 & 0.35330 \\
\hline 617 & 0.39400 & 587 & 0.38011 & 771 & 0.36570 & 802 & 0.35317 \\
\hline 740 & 0.39289 & 734 & 0.37997 & 706 & 0.36566 & 598 & 0.35299 \\
\hline 665 & 0.39280 & 684 & 0.37992 & 648 & 0.36544 & 782 & 0.35278 \\
\hline 529 & 0.39280 & 666 & 0.37990 & 745 & 0.36525 & 717. & 0.35274 \\
\hline 577 & $0: 39267$ & 692 & 0.37978 & 716 & 0.36495 & 812 & 0.35253 \\
\hline 67.4 & 0.39246 & 608 & 0.37734 & 588 & 0.36438 & 668 & 0.35205 \\
\hline 723 & 0.39235 & 705 & 0.37712 & 726 & 0.36285 & 765 & 0.35187 \\
\hline 741 & 0.39166 & 618 & 0.37656 & 772 & 0.36275 & 774 & 0.35163 \\
\hline 627 & 0.39137 & 760 & 0.37638 & 499 & 0.36243 & 830 & 0.35102 \\
\hline 656 & 0.38897 & 715 & 0.37635 & 764 & 0.36013 & 727. & 0.35084 \\
\hline 539 & 0.38847 & 559 & 0.37553 & 609 & 0.36002 & 822 & 0.35064 \\
\hline 742 & 0.38804 & 578 & 0.37539 & 736 & 0.35944 & 831 & 0.35014 \\
\hline 733 & 0.38804 & 761 & 0.37530 & 686 & 0.35939 & 649 & 0.34965 \\
\hline 683 & 0.38798 & 753 & 0.37514 & 619 & 0.35935 & 756 & 0.34911 \\
\hline 704 & 0.38735 & 489 & 0.37479 & 658 & 0.35923 & 803 & 0.34877 \\
\hline 637. & 0.38709 & 744. & 0.37452 & 755 & 0.35904 & 589 & 0.34871 \\
\hline 479 & 0.38681 & 693 & 0.37432 & 695 & 0.35833 & 696 & 0.34846 \\
\hline 714 & 0.38651 & 657 & 0.37428 & 579 & 0.35833 & 783 & 0.34840 \\
\hline 568 & 0.38580 & 628 & 0.37426 & 773 & 0.35799 & 813 & 0.34816 \\
\hline 750 & 0.38520 & 725 & 0.37405 & 629 & 0.35734 & 737 & 0.34775 \\
\hline 399 & 0.38479 & 762 & 0.37211 & 677 & 0.35724 & 687 & 0.34771 \\
\hline 690. & 0.38432 & 638 & 0.37051 & 800 & 0.35681 & 832 & 0.34755 \\
\hline 751 & 0.38404 & 735 & 0.37031 & 780 & 0.35641 & 840 & 0.34670 \\
\hline 724 & 0.38402 & 685 & 0.37026 & 810 & 0.35615 & 823 & 0.34633 \\
\hline 691 & 0.38317 & 676 & 0.36995 & 801 & 0.35589 & 841 & 0.34586 \\
\hline 549 & 0.38264 & 754 & 0.36783 & 781 & 0.35549 & 790 & 0.34574 \\
\hline
\end{tabular}


Table II contd.

\begin{tabular}{|c|c|c|c|c|c|c|c|}
\hline (hkl) & dhke & (hke) & dhke & (hke) & dhke & (hkl) & dhke \\
\hline 791 & 0.34490 & 776 & 0.33516 & 855 & 0.32278 & 931 & 0.31245 \\
\hline-659 & 0.34419 & 861 & 0.33444 & 873 & 0.32201 & 891 & 0.31239 \\
\hline 678 & 0.34409 & -853 & 0.33432 & 768 & 0.32168 & 875 & 0.31166 \\
\hline 775 & 0.34393 & 844 & 0.33388 & 880 & 0.32086 & 903 & 0.31148 \\
\hline 747 & 0.34355 & 825 & 0.33355 & 881 & 0.32019 & 847 & 0.31138 \\
\hline 842 & 0.34336 & 794 & 0.33302 & 749 & 0.31984 & 913 & 0.31104 \\
\hline 833 & 0.34336 & 767 & 0.33235 & 846 & 0.31969 & 769 & 0.31074 \\
\hline 804 & 0.34288 & 862 & 0.33218 & 796 & 0.31893 & 797 & 0.31068 \\
\hline 784 & 0.34252 & 748 & 0.33180 & 807 & 0.31864 & 884 & 0.31062 \\
\hline 766 & 0.34251 & 835 & 0.33089 & 787 & 0.31836 & 932 & 0.31061 \\
\hline 792 & 0.34242 & 679 & 0.33080 & 882 & 0.31821 & 866 & 0.31061 \\
\hline 814 & 0.34230 & .854 & 0.32912 & 817 & 0.31817 & 892 & 0.31054 \\
\hline 850 & 0.34138 & 863 & 0.32851 & 865 & 0.31754 & 940 & 0.31000 \\
\hline 708 & 0.34063 & 870 & 0.32831 & 874 & 0.31736 & 923 & 0.30974 \\
\hline 851 & 0.34058 & 709 & 0.32773 & 900 & 0.31717 & 941 & 0.30940 \\
\hline 824 & 0.34056 & 871 & 0.32759 & 827 & 0.31678 & 808 & 0.30920 \\
\hline 718 & 0.34006 & 806 & 0.32756 & 910 & 0.31670 & 788 & 0.30894 \\
\hline 843 & 0.33931 & 845 & 0.32727 & 901 & 0.31652 & 818 & 0.30877 \\
\hline 599 & 0.33869 & 786 & 0.32725 & 911 & 0.31606 & 942 & 0.30761 \\
\hline 793 & 0.33841 & 719 & 0.32722 & 759 & 0.31565 & 933 & 0.30760 \\
\hline 757 & 0.33837 & 758 & 0.32713 & 778 & 0.31557 & 893 & 0.30754 \\
\hline 728 & 0.33836 & 816 & 0.32705 & 856 & 0.31551 & 857 & 0.30751 \\
\hline 852 & 0.33819 & 698 & 0.32659 & 920 & 0.31533 & 828 & 0.30750 \\
\hline 669 & 0.33786 & 795 & 0.32645 & 699 & 0.31517 & 904 & 0.30726 \\
\hline 697 & 0.33777 & 729 & 0.32570 & 883 & 0.31498 & 914 & 0.30684 \\
\hline 834 & 0.33773 & 777 & 0.32563 & 921 & 0.31469 & 950 & 0.30618 \\
\hline 805 & 0.33573 & 826 & 0.32554 & 902 & 0.31460 & 951 & 0.30560 \\
\hline 738 & 0.33558 & 872 & 0.32547 & 837 & 0.31450 & 924 & 0.30559 \\
\hline 688 & 0.33554 & 864 & 0.32357 & 912 & 0.31415 & 838 & 0.30541 \\
\hline 785 & 0.33539 & 739 & 0.32322 & 930 & 0.31308 & 885 & 0.30527 \\
\hline 860 & 0.33520 & 689 & 0.32319 & 890 & 0.31301 & 779 & 0.30523 \\
\hline 815 & 0.33518 & 836 & 0.32306 & 922 & 0.31281 & 876 & 0.30510 \\
\hline
\end{tabular}


Table II contd:

\begin{tabular}{|c|c|c|c|c|c|c|c|}
\hline (hke) & $\mathrm{d}_{\mathrm{hk} \ell}$ & $(\mathrm{hk \ell} \ell)$ & $\mathrm{dhk \ell}$ & $(\mathrm{hk} \ell)$ & dhk $\ell$ & (hkel) & ${ }_{\mathrm{d}}^{\mathrm{hk \ell}}$ \\
\hline 943 & 0.30469 & 868. & 0.29482 & 984 & 0.28340 & 969 & 0.26460 \\
\hline 952 & 0.30387 & 926 & 0.29460 & 966. & 0.28339 & 997 & 0.26456 \\
\hline 934 & 0.30354 & 972 & 0.29454 & 992 & 0.28334 & 988 & 0.26348 \\
\hline 894 & 0.30348 & 849 & 0.29341 & 908. & 0.28232 & 979 & 0.26117 \\
\hline 867. & 0.30097 & 964 & 0.29314 & 918 & 0.28200 & 998 & 0.25908 \\
\hline 848 & 0.30256 & 799 & 0.29282 & 879 & 0.28200 & 989 & 0.25737 \\
\hline 905 & 0.30208 & 936 & 0.29276 & 993 & 0.28106 & 999 & 0.25326 \\
\hline 798 & 0.30191 & 896 & 0.29270 & 957 & 0.28103 & & \\
\hline 960 & 0.30170 & 955 & 0.29255 & 928. & 0.28103 & & \\
\hline 915 & 0.30168 & 887 & 0.29226 & 938 & 0.27943 & & \\
\hline 961 & 0.30114 & 973 & 0.29198 & 898 & 0.27938 & & \\
\hline 953 & 0.30106 & 980 & 0.29112 & 985 & 0.27932 & & \\
\hline 944 & 0.30074 & 981 & 0.29062 & 976 & 0.27919 & & \\
\hline 925 & 0.30049 & 946. & 0.29024 & 994 & 0.27794 & & \\
\hline 962 & 0.29949 & 859 & 0.29016 & 967. & 0.27755 & & \\
\hline 809 & 0.29946 & 878 & 0.29010 & 948 & 0.27724 & $\because$ & \\
\hline 789 & 0.29922 & 907 & 0.28946 & 889 & 0.27724 & & $\because$ \\
\hline 886 & 0.29910 & 982 & 0.28913 & 909 & 0.27485 & ? & \\
\hline 819 & 0.29907 & 917 & 0.28910 & 986 & 0.27457 & & $\therefore$ \\
\hline 858 & 0.29900 & 965 & 0.28863 & 919 & 0.27455 & & \\
\hline 935 & 0.29854 & 97.4 & 0.28849 & 958 & 0.27449 & $\therefore$ & $\therefore$ \\
\hline 895 & 0.29848 & 927 & 0.28806 & 995 & 0.27409 & $\vdots$ & $\therefore$ \\
\hline 829 & 0.29791 & 956 & 0.28710 & 929. & 0.27365 & & \\
\hline 877 & 0.29785 & 983 & 0.28670 & 977 & 0.27360 & & $\therefore$ \\
\hline 954 & 0.29724 & 937 & 0.28634 & 939 & 0.27217 & & \\
\hline 963 & 0.29679 & 869 & 0.28633 & 89.9 & 0.27213 & & $\therefore$ \\
\hline 970 & 0.29664 & 897 & 0.28628 & 968 & 0.27125 & & . \\
\hline 971 & 0.29612 & 990 & 0.28521 & 949 & 0.27015 & & \\
\hline 906 & 0.29609 & 888 & 0.28492 & 996 & 0.26960 & & \\
\hline 839 & 0.29601 & 991 & 0.28474 & 987 & 0.26925 & & \\
\hline 945 & 0.29587 & 975 & 0.28419 & 959 & 0.26761 & & \\
\hline 916 & 0.29572 & 947 & 0.28398 & 978 & 0.26756 & & \\
\hline
\end{tabular}




\section{Table III}

Interplanar Spacings, dhk , in Angstroms for $\mathrm{U}_{3} \mathrm{Si}$ at $25^{\circ} \mathrm{C}$

Arranged in (hke) Order.

(without regard for extinctions, using $a_{0}=6.0290 \AA$ and $c_{0}=8.6963 \AA$

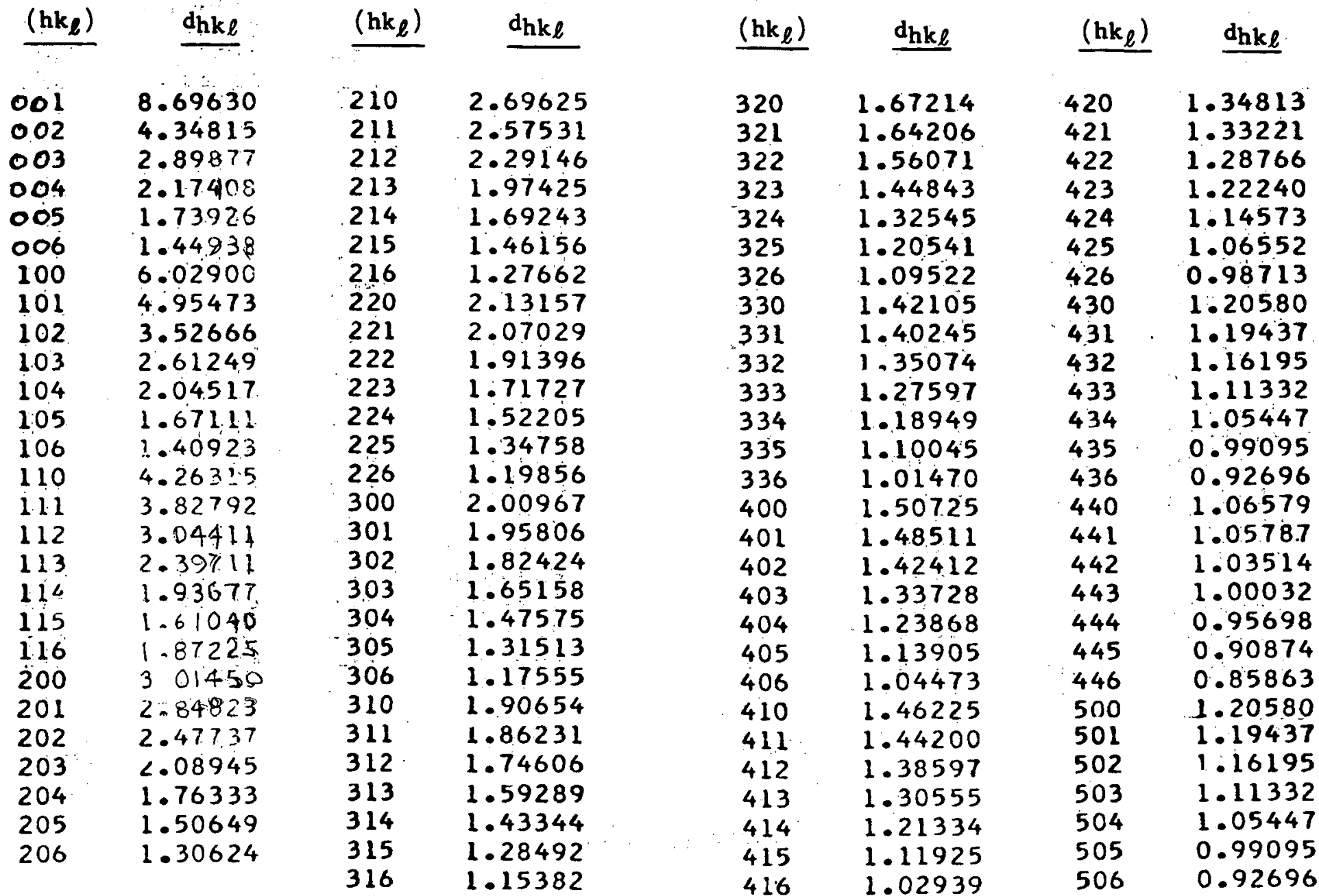


Table III contd.

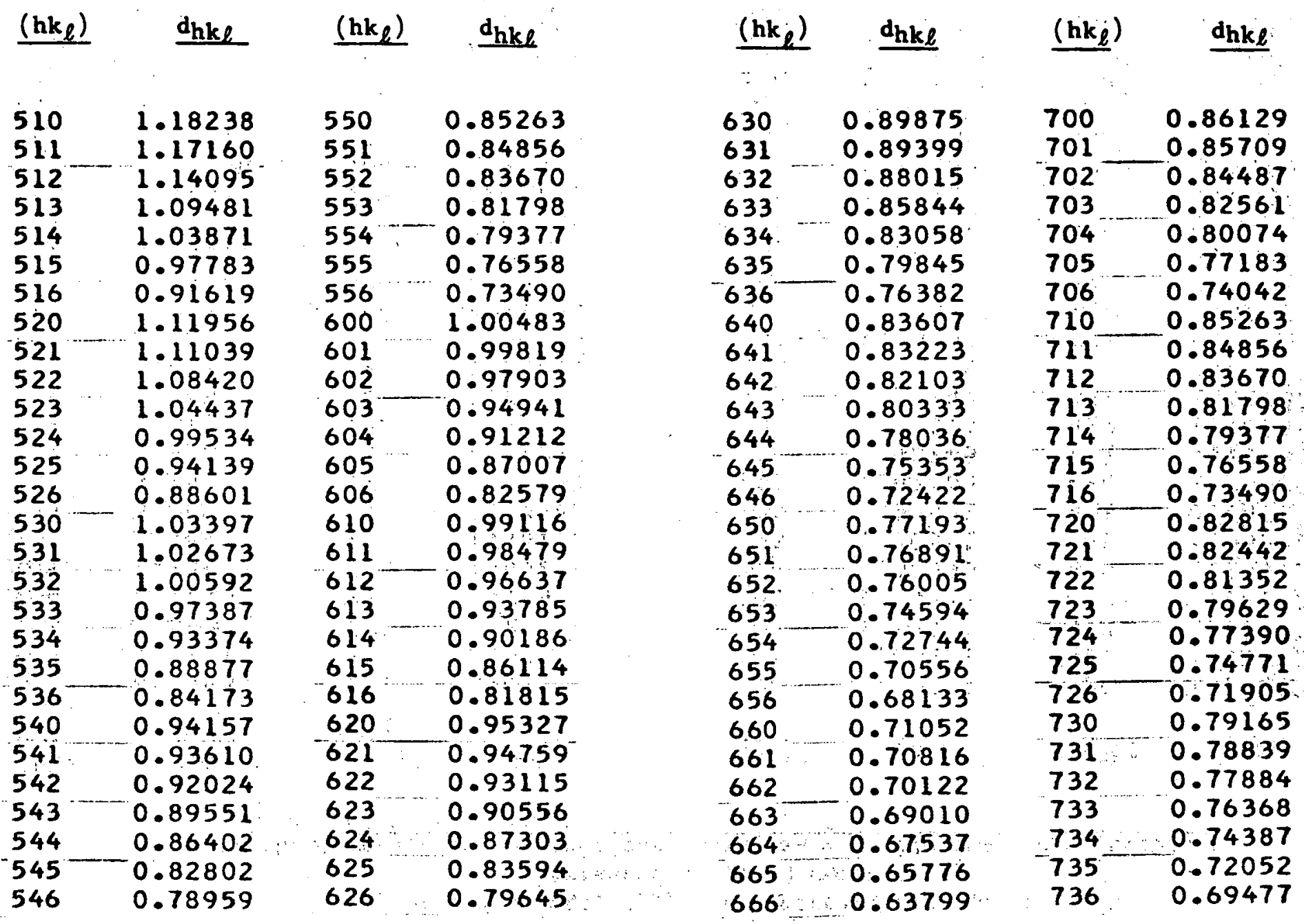


Table III contd.

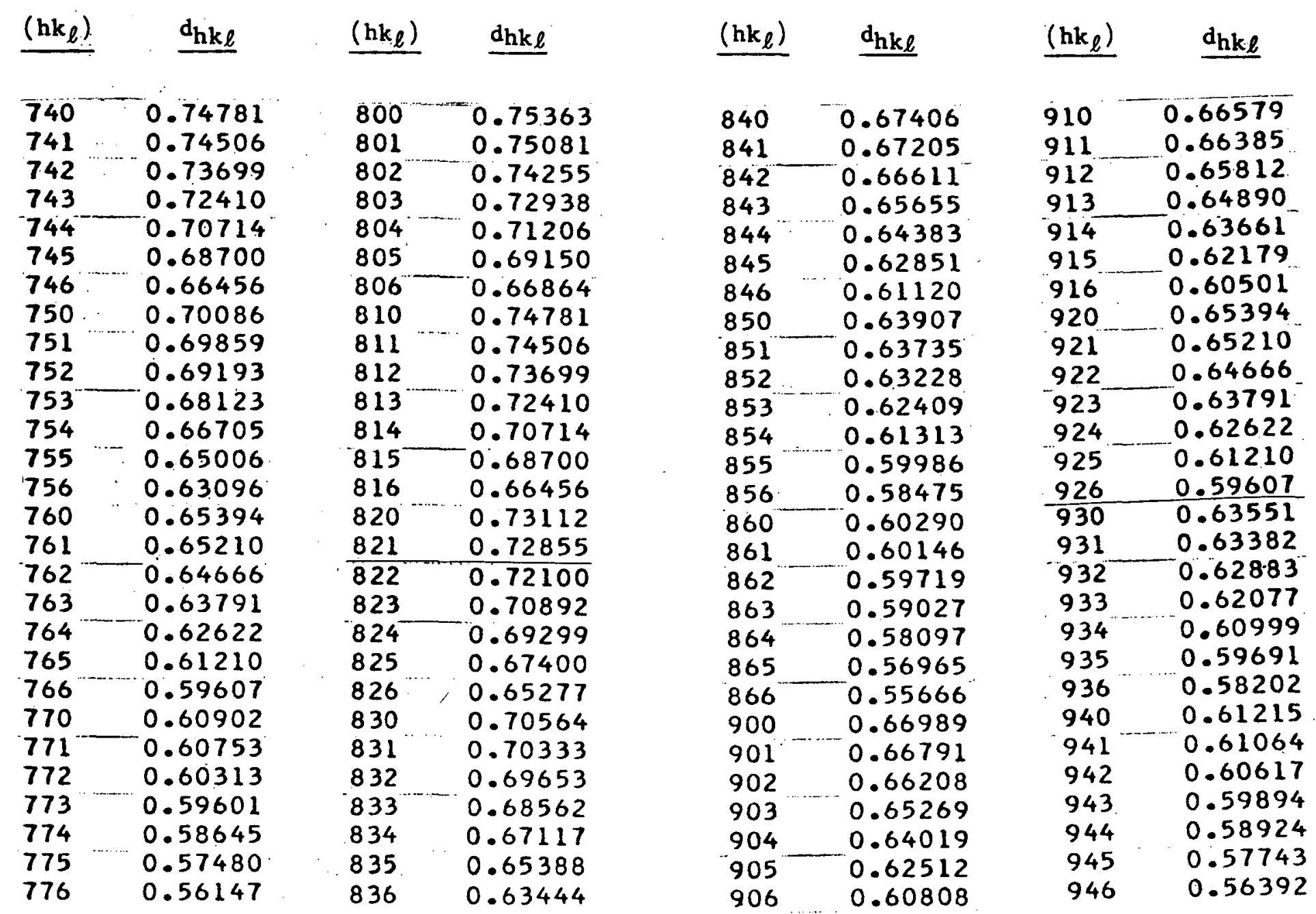


Interplanar Spacing, dhkl, in Angstroms for $\mathrm{U}_{3} \mathrm{Si}$ at $25^{\circ} \mathrm{C}$

dhke order
(without regard for extinctions, using $a_{0}=6.0090 \AA$ and $c_{0}=8.6963 \AA$ )

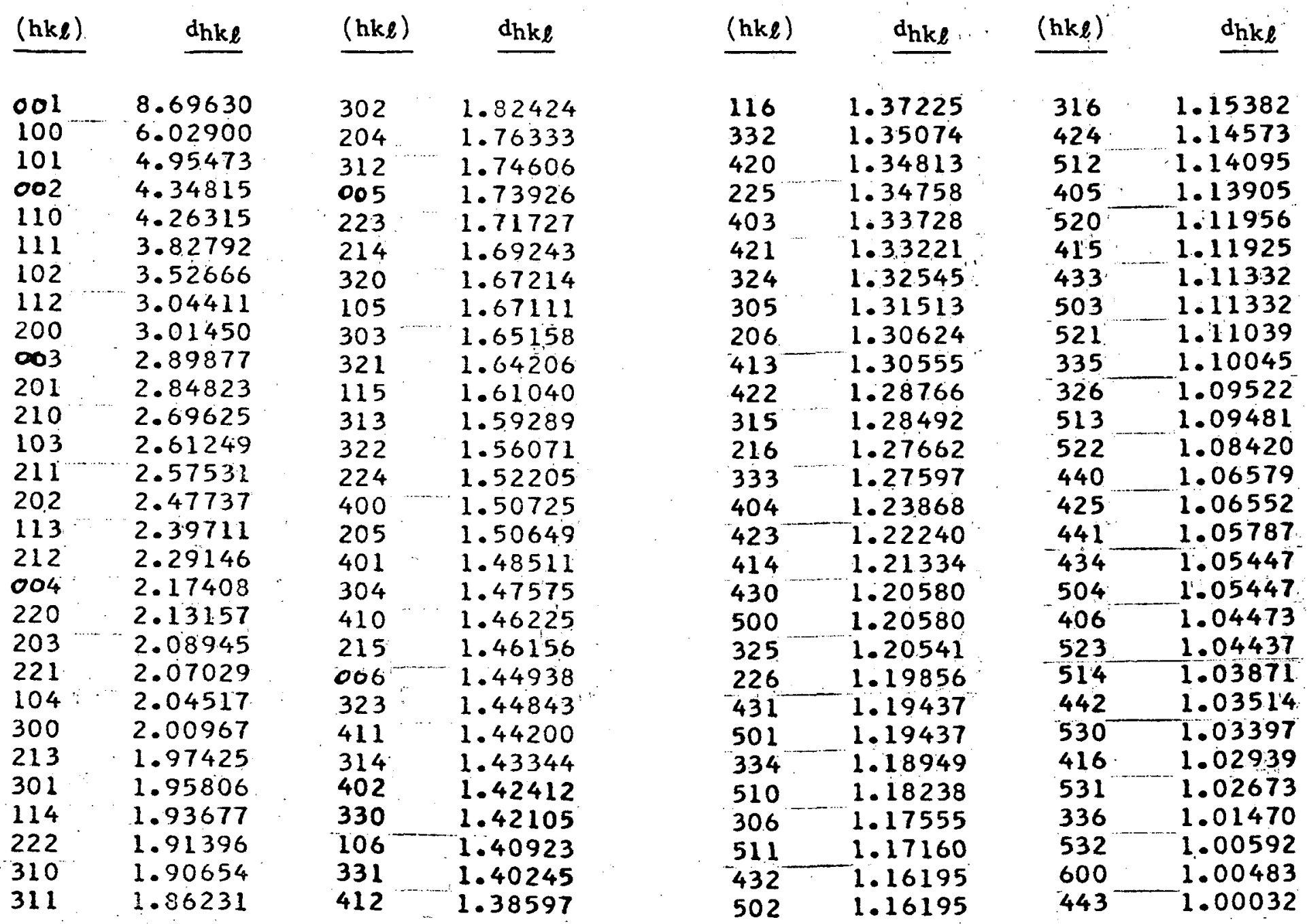


Table IV contd.

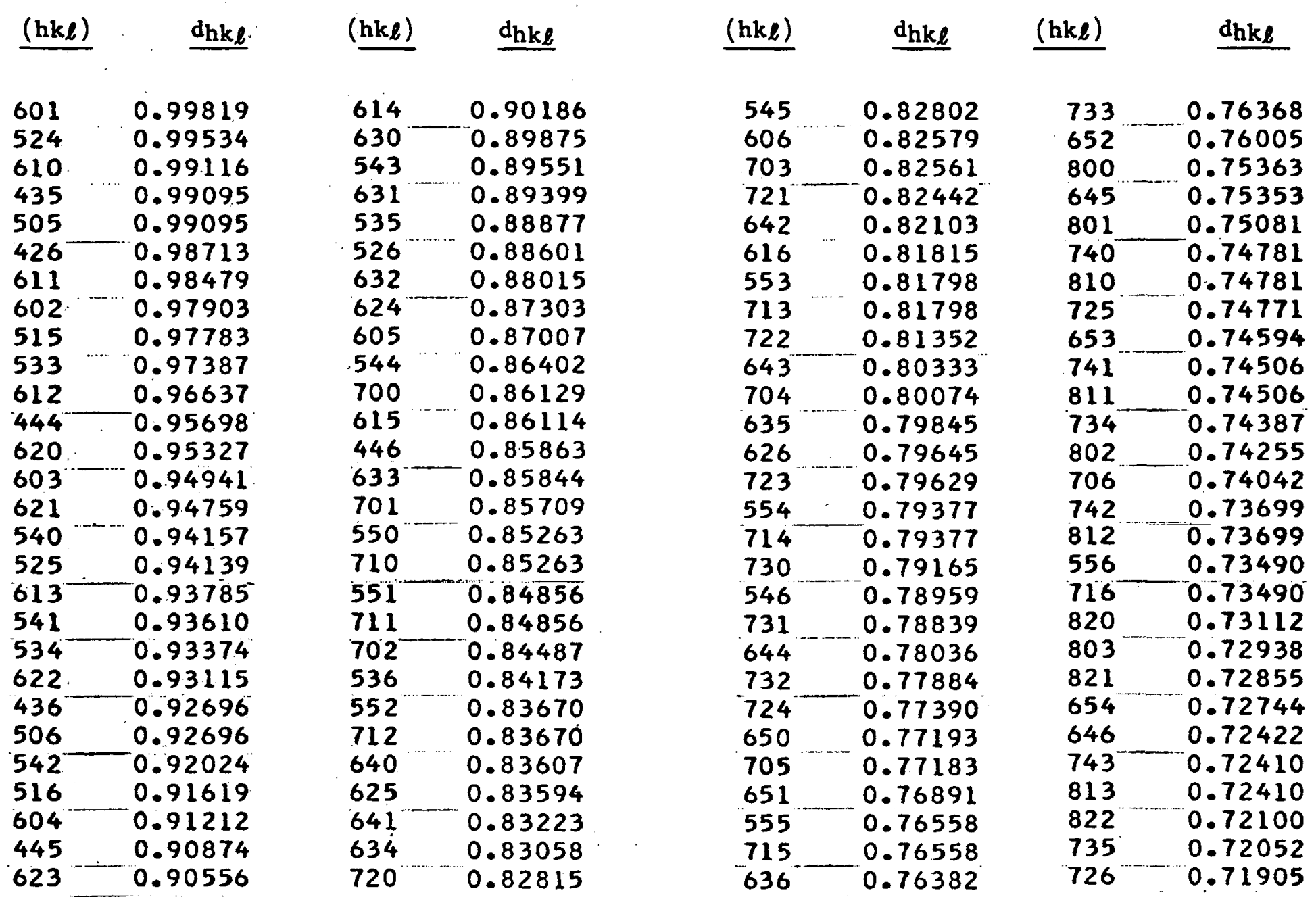


Table IV contd:

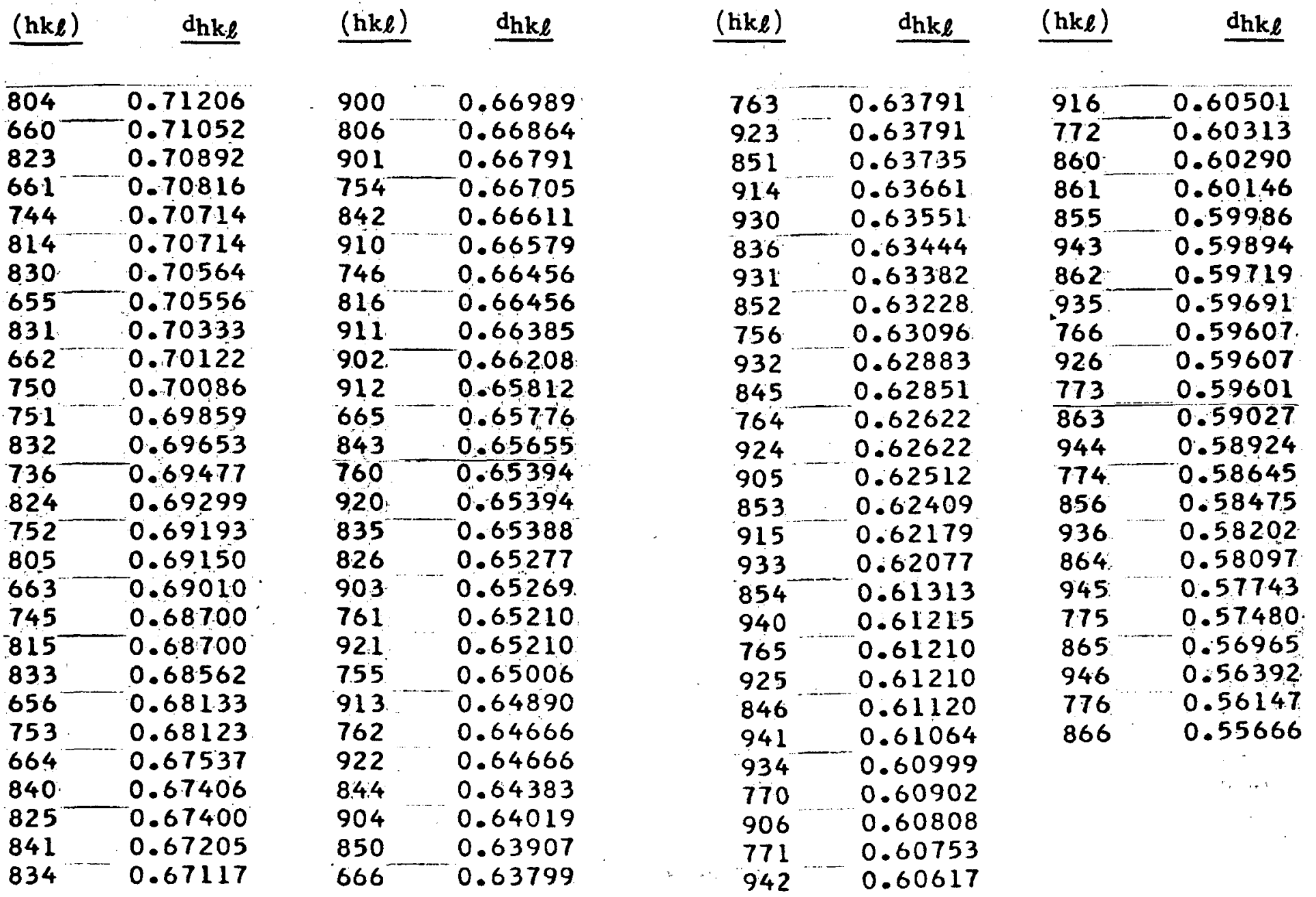


For the tetragonal case, $a=b$ and for the cubic case, $a=b=c$.

Table $V$ lists the angles between the (hkl) plane and the three cardinal planes $(100),(010)$ and $(001)$ for $\mathrm{U}_{3} \mathrm{Si}$ using the same $25^{\circ} \mathrm{C}$ lattice constants as for the ¿ spacings in Tables III and IV. Again, the table has been abbreviated since $h$ and $k$ may be interchanged if the angular values for the reference planes (100) and (010) are also interchanged. Since the angle between the poles of two planes is the same as the angle between the planes themselves, this information can be used to plot a pole figure for $U_{3} S i$. Figure 1 shows a standard (001) projection for $\mathrm{U}_{3} \mathrm{Si}$ plotted without regard for extinctions from the above data. Figure 2 shows a similar plot of the (001) projection for alpha-uranium prepared by Muêller and Knott 5 of ANL. These two stereographic projections and the well known Polar and Wulff provide the basic tools for uranium crystallographic manipulations. With these it is possible to identify twin planes and slip planes from their traces and to determine crystal orientation relationships wen crystal matrix and precipitate diffraction patterns are superimposed. Other manipulations are possible but these serve to illustrate why the pole figures are necessary. Chapter two of Barrett ${ }^{3}$ gives an excellent introduction to stereographic projection and its uses. Since a stereographic projection of alpha-uranium was al ready available, it was not necessary to calculate the interplanar angles to obtain one. However, it was desirable to know the angles between the uranium twin planes and the three cardinal planes. Calculations for all of these angles were not avallable, especially for some which do not have rational indices. Table VI is a compilation of the calculated values for angles between the uranium twin planes. (hke) and the three cardinal planes (100), (010) and (001). Again, since the angle between planes equals the angles between poles, they may be conveniently plotted on a stereographic projection. Table VII is a listing of the calculated values of angles between the uranium twin direction [hkl] and the three cardinal directions [100], [010] and [001]. Appendix III of Barrett ${ }^{3}$ lists a formula for calculating this angle, which in modified symbols is as follows:

$$
\cos \phi=\frac{h_{1} h_{2} a_{i 2}^{2}+k_{1} k_{2} b^{2}+l_{1} l_{2} c^{2}}{\sqrt{h_{1}^{2} a^{2}+k_{1}^{2} b^{2}+l_{1}^{2} c^{2}}: \sqrt{h_{2}^{2} a^{2}+k_{2}^{2} b^{2}+l_{2}^{2} c^{2}}}
$$

Tables VI and VII are based on the $25^{\circ} \mathrm{C}$ lattice parameters ${ }^{2}$ for uranium of $a_{0}=2.8545 \AA, b_{0}=5.8681 \AA$, and $c_{o}=4.9566 \AA$. The planes and directions represented in these two tables reflect the possible twinning modes for uranium given by A. G. Crocker.6.

AGEING STUDIES OF URANIUM-SILICON ALLOYS WITH THE ELECTRON MICROSCOPE.

Heat Treatment of Samples

Samples of uranium containing 330,530 , and $1500 \mathrm{ppm}$ of silicon were solutionannealed in the beta phase for periods of 24 to 48 hours. The samples were then quenched in water to retain the silicon in solid soltion. All of the 
Interplanar Angles for $\mathrm{U}_{3} \mathrm{Si}$ at $25^{\circ} \mathrm{C}$ (using $a_{0}=6.0290 \AA$ and $c_{0}=8.6963 \AA$ )

Angle between Planes

\begin{tabular}{|c|c|c|c|}
\hline (hkl) & $(100)$ & $\frac{\text { Degrees }}{(010)}$ & $(001)$ \\
\hline 001 & $90: 000$ & 90.000 & 0.000 \\
\hline$\infty 2$ & 90.000 & 90.000 & 0.000 \\
\hline 003 & 90.000 & 90.000 & 0.000 \\
\hline 004 & 90.000 & 90.000 & 000 \\
\hline 005 & 90.000 & 90.000 & 0.000 \\
\hline 006 & 90.000 & 90.000 & 0.000 \\
\hline 100 & 0.000 & 90.000 & 90.000 \\
\hline 101 & 34.733 & 90.000 & 55.267 \\
\hline 102 & 54.201 & 90.000 & 35.800 \\
\hline 103 & 64.322 & 90.000 & 25.679 \\
\hline 104 & 70.171 & 90.000 & 19.830 \\
\hline 105 & 73.908 & 90.000 & 16.092 \\
\hline 106 & 76.483 & 90.000 & 13.518 \\
\hline $110^{\circ}$ & 45.000 & 45.000 & 90.000 \\
\hline 111 & 50.586 & 50.586 & 63.885 \\
\hline 112 & 59.675 & 59.675 & 45.566 \\
\hline 113 & 66.572 & 66.572 & 34.214 \\
\hline 114 & 71.262 & 71.262 & 27.020 \\
\hline 115 & 74.508 & 74.508 & 22.194 \\
\hline 116 & 76.844 & 76.844 & 18.777 \\
\hline 200 & 0.000 & 90.000 & 90.000 \\
\hline 201 & 19.119 & 90.000 & 70.882 \\
\hline 202 & 34.733 & 90.000 & 55.267 \\
\hline 203 & 46.121 & 90.000 & 43.879 \\
\hline 204 & 54.201 & 90.000 & 35.800 \\
\hline 205 & 60.017 & 90.000 & 29.984 \\
\hline 206 & 64.322 & 90.000 & .679 \\
\hline
\end{tabular}

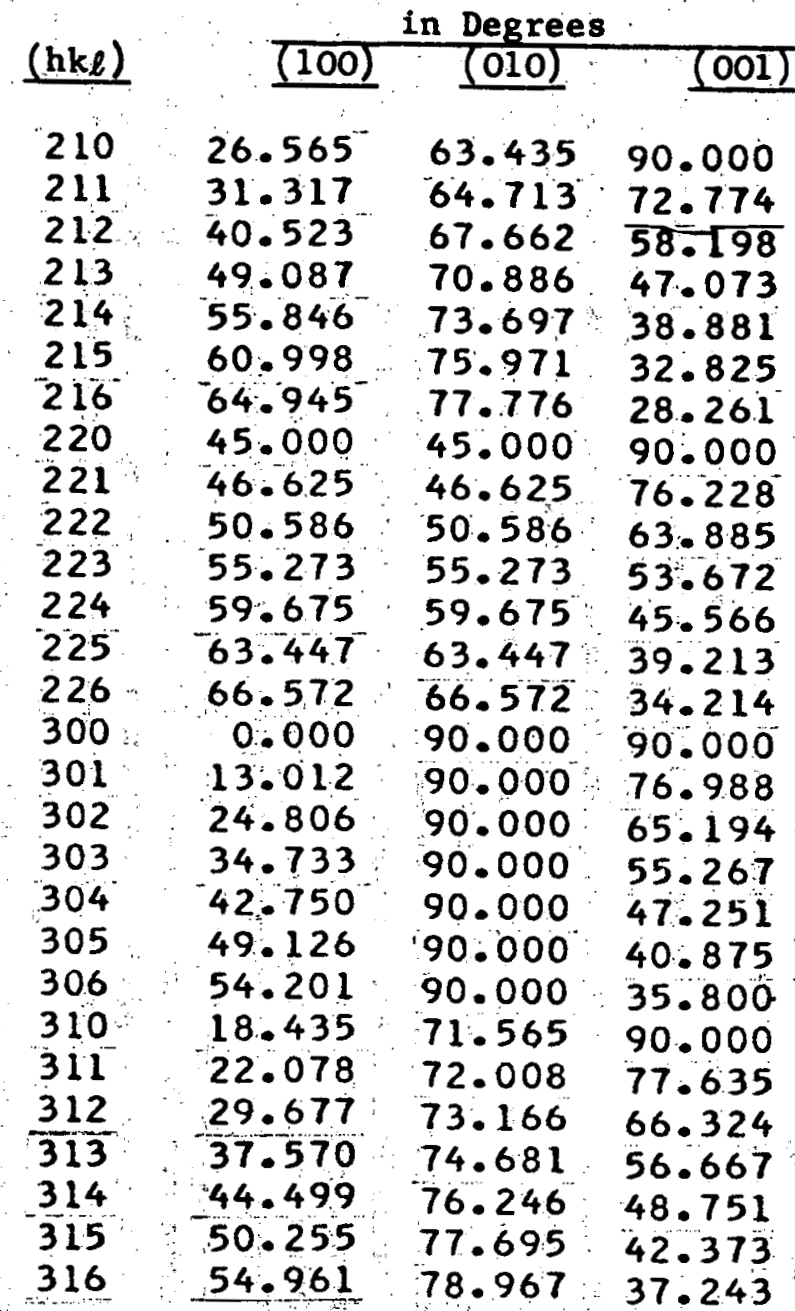


Table V contd.

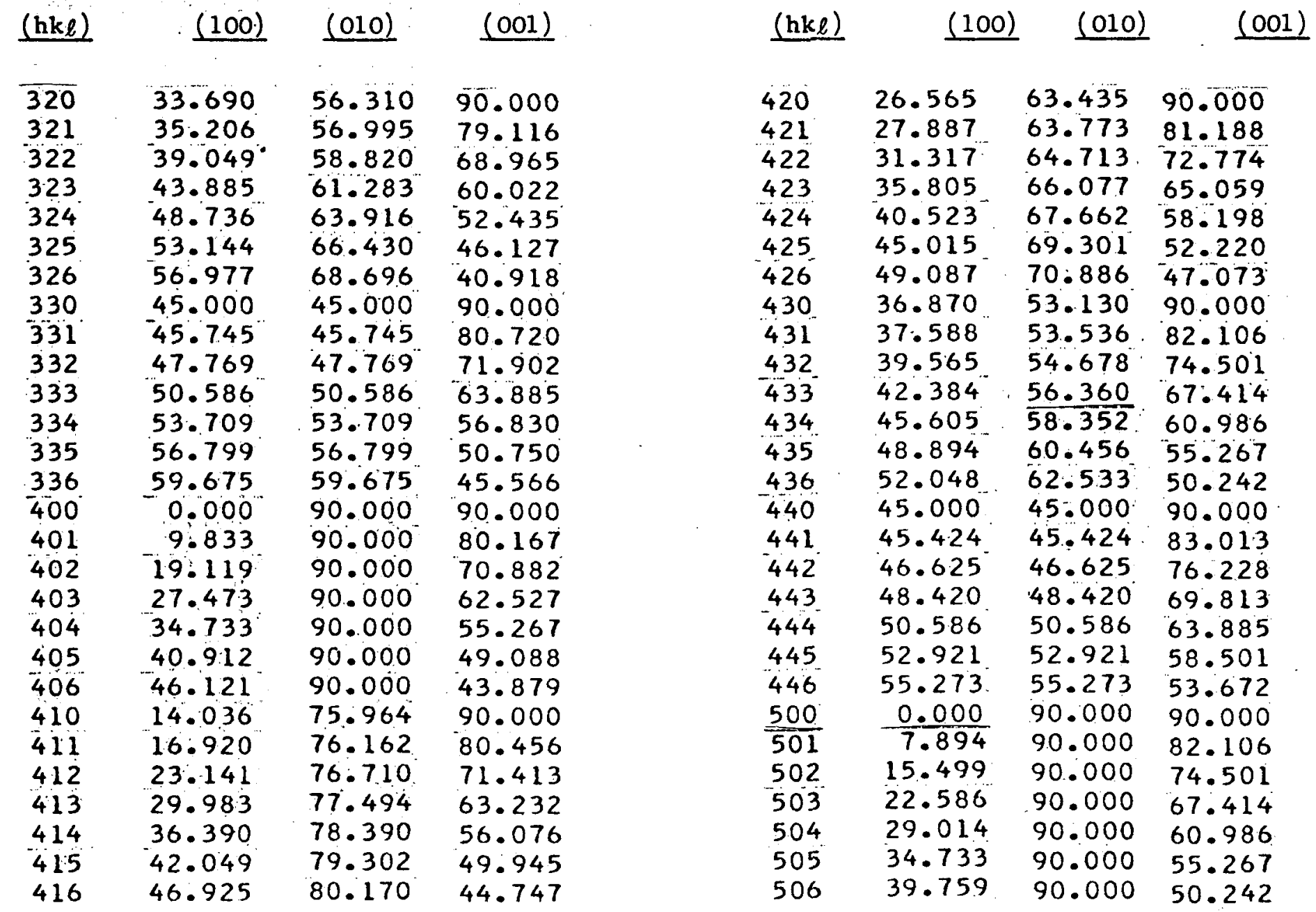


Table V contd.

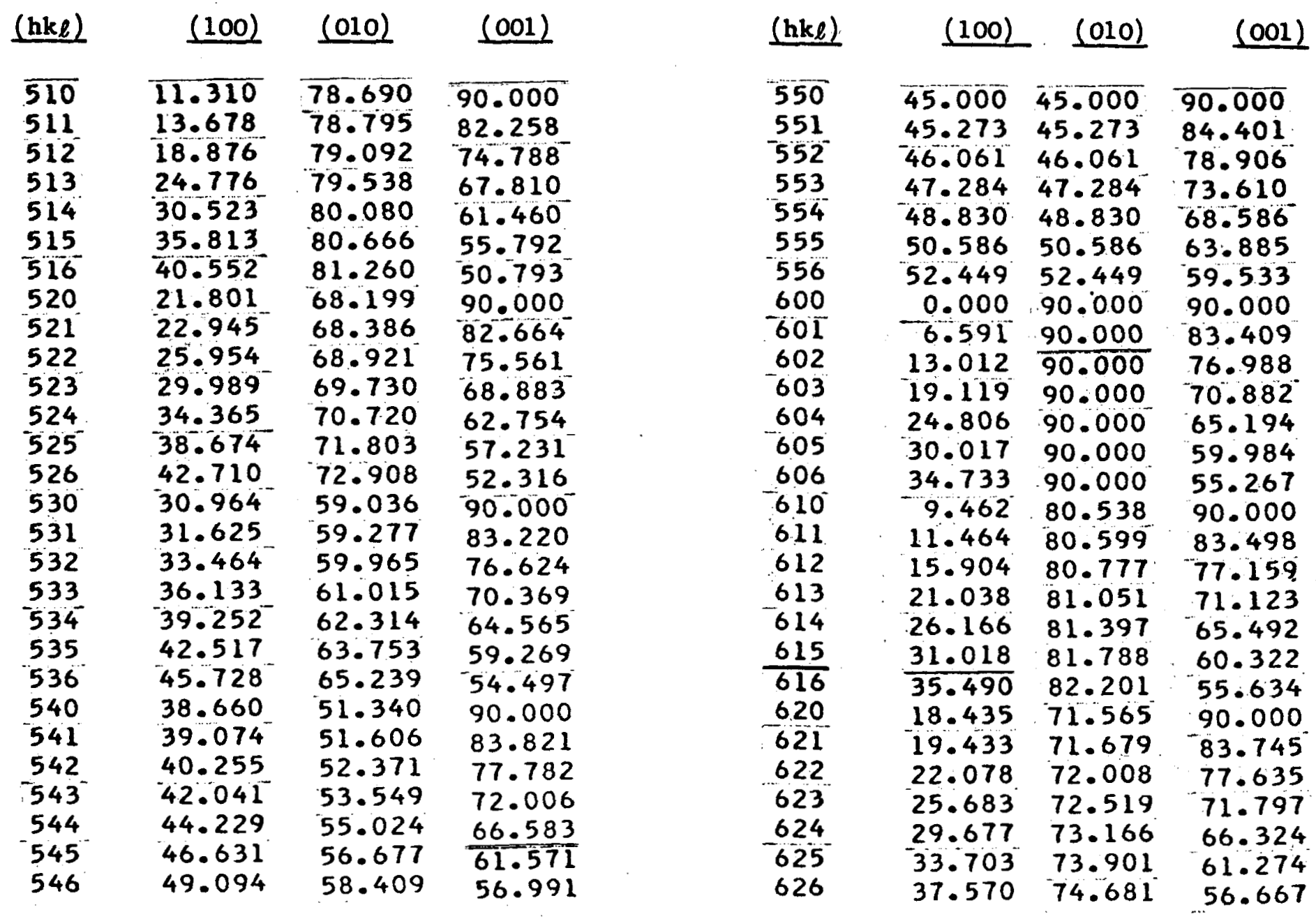


Table V contd.

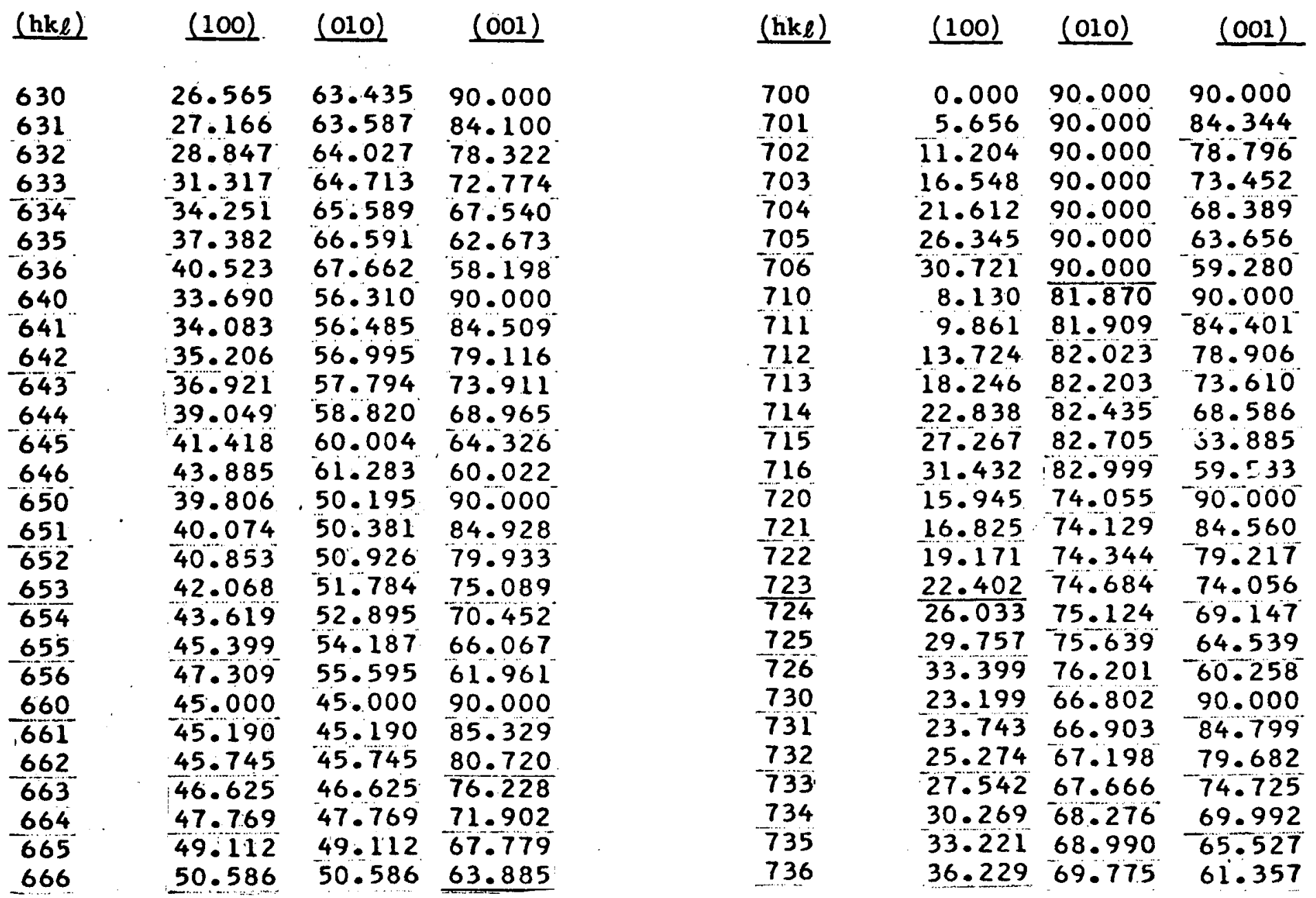


Table V contd.

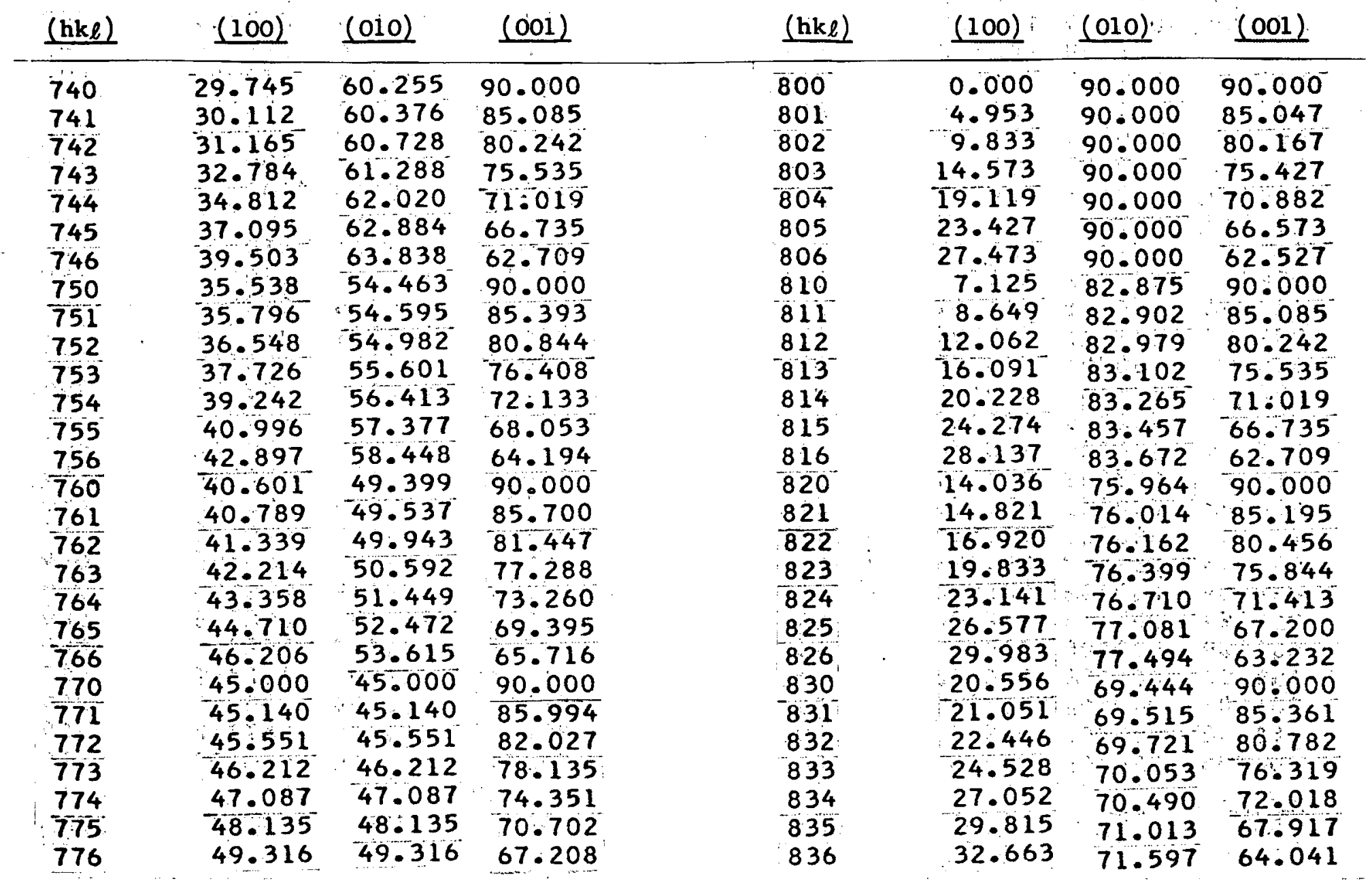


Table V contd.

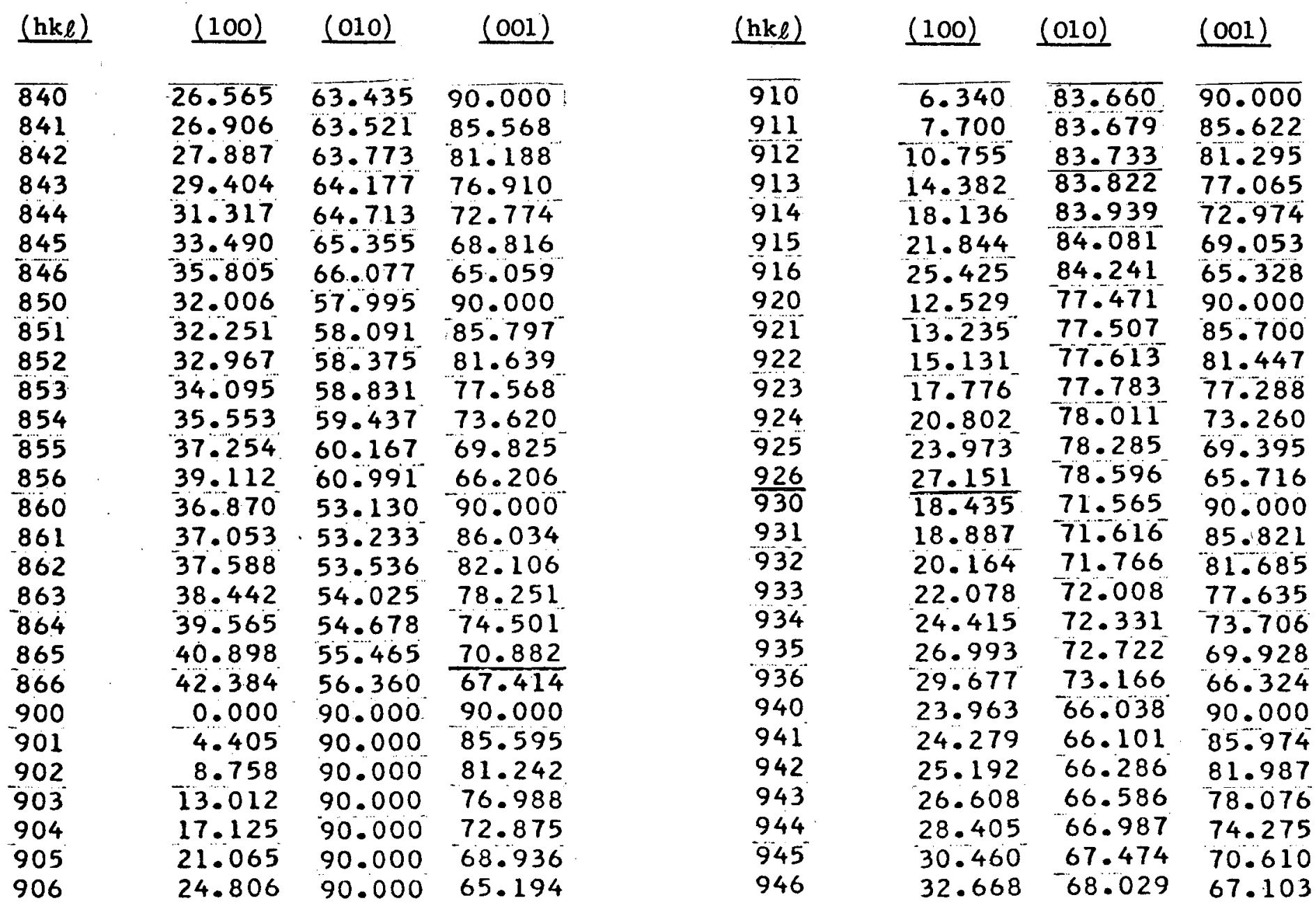


The Angle between the Twin Planes (hkl) and the (100), (010), and (001) Planes for Alpha-Uranium at $25^{\circ} \mathrm{C}$ with $a_{0}=2.8545 \AA, b_{0}=5.8681 \AA$ and $c_{0}=4.9566 \AA$

Angle between Planes

in Degrees

$\underline{(\text { hke) }}$

\begin{tabular}{lll}
1 & 1 & 1 \\
1 & 1 & 2 \\
0 & 2 & 1 \\
0 & 0 & 1 \\
1 & 1 & 0 \\
\hline 4 & 2 & 1 \\
\hline 0 & 1 & 1 \\
2 & 6 & 1
\end{tabular}

37.011
51.347
90.000
90.000
25.940
15.782
90.000
56.088
73.851
90.000
60.876
90.000
29.479
51.347
57.486
74.454
60.876
20.653
61.725
66.988
39.800
26.368
80.462
74.454
8.044
8.193
55.579

55.579

$(010)$

\begin{tabular}{ll}
767.143 & 62.622 \\
72.312 & 43.994 \\
30.623 & 59.377 \\
90.000 & 0.000 \\
64.060 & 90.000 \\
76.465 & 82.037 \\
49.813 & 40.187 \\
35.492 & 80.755 \\
18.721 & 80.783 \\
0.000 & 90.000 \\
76.305 & 32.768 \\
60.616 & 29.385 \\
64.947 & 75.483 \\
72.312 & 43.994 \\
38.336 & 71.968 \\
24.130 & 72.019 \\
76.305 & 32.768 \\
76.845 & 74.369 \\
46.267 & 56.933 \\
79.038 & 25.769 \\
51.473 & 81.519 \\
77.414 & 67.233 \\
43.493 & 48.086 \\
24.130 & 72.019 \\
\hline 83.084 & 85.913 \\
90.000 & 81.807 \\
\hline 34.421 & 90.000
\end{tabular}

Angle between Planes

$\underline{(h k \ell)}$

in Degrees

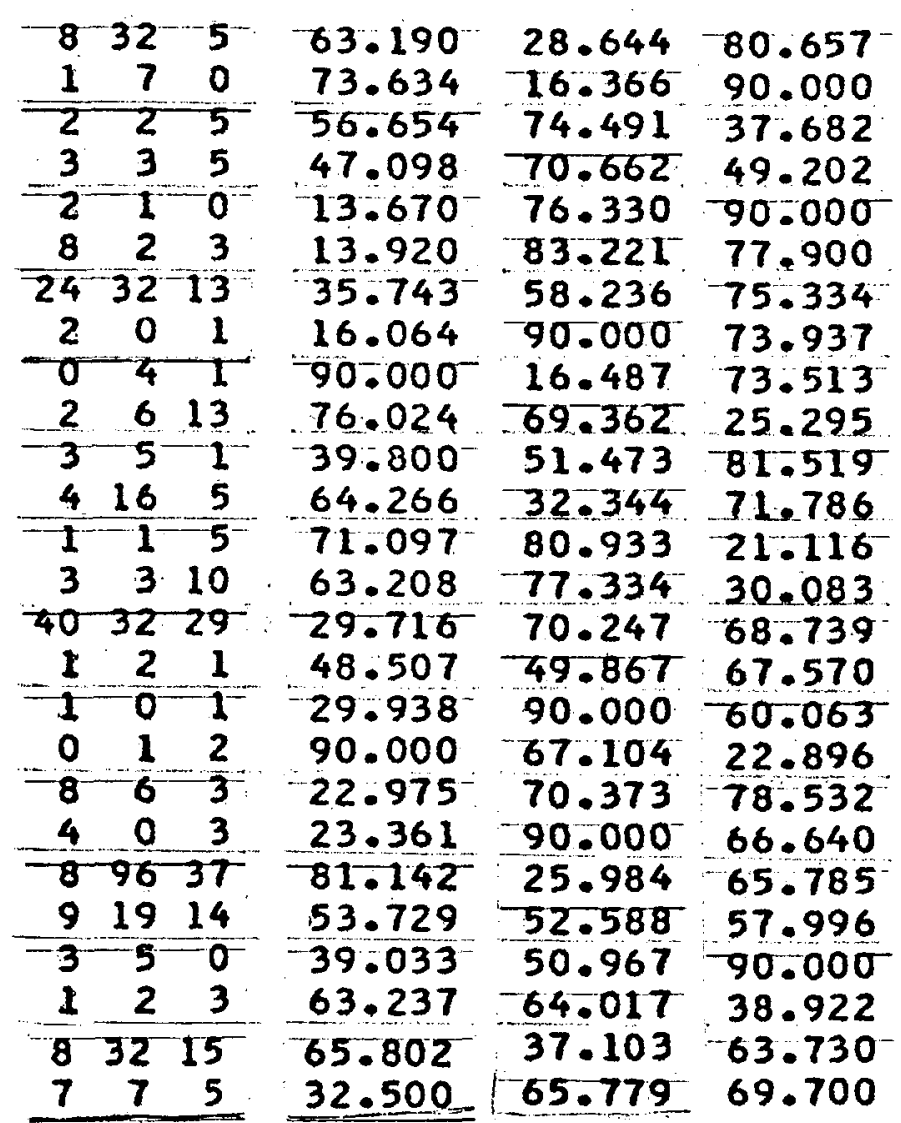




\section{Table VII}

The Angle between the Twinning Directions [hkl] and the [100],

[010] and [001] Directions for Alpha-Uranium at $25^{\circ} \mathrm{C}$ with

$a_{0}=2.8545 \AA, b_{0}=5.8681 \AA$ and $c_{0}=4.9566 \AA$

Angle between Directions

in Degrees

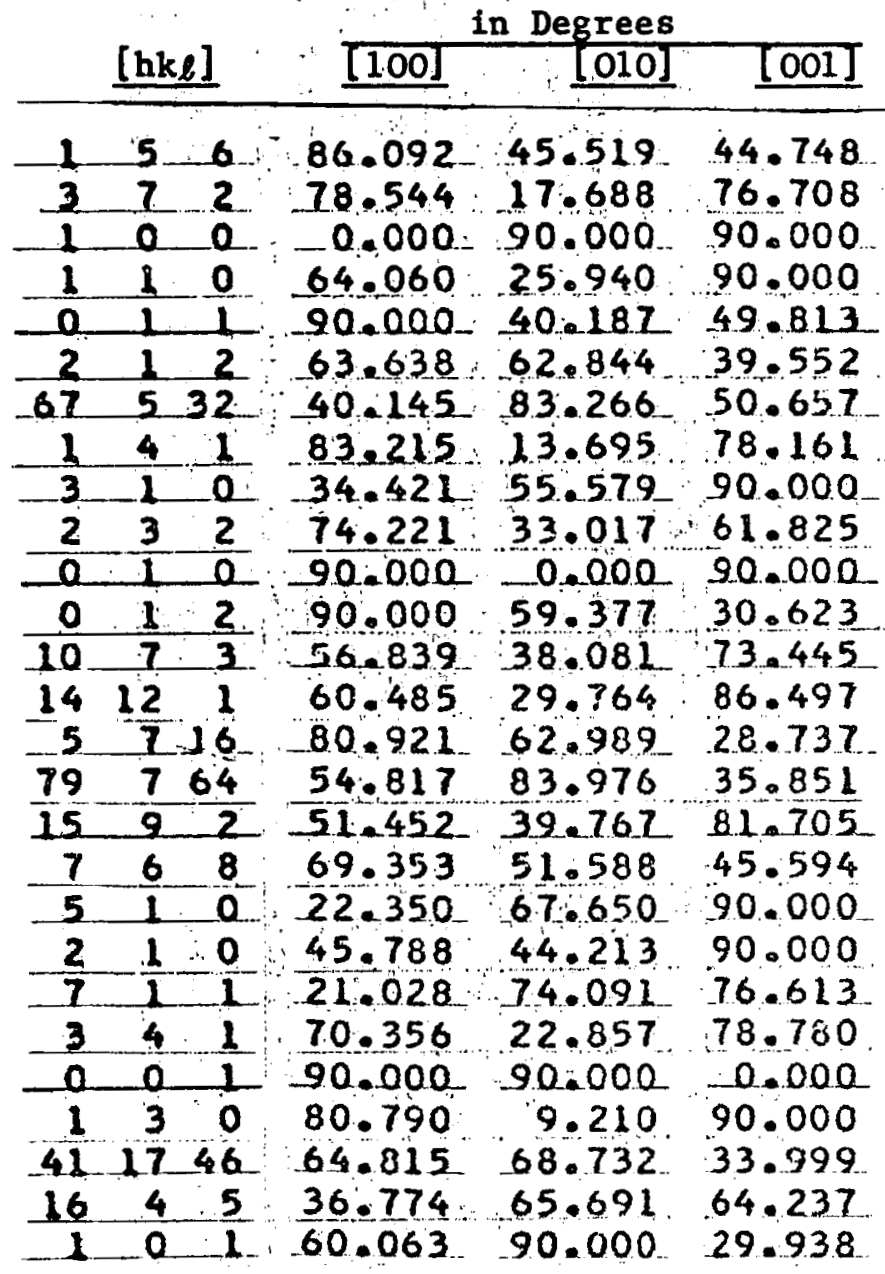

Angle between Directions in Degrees

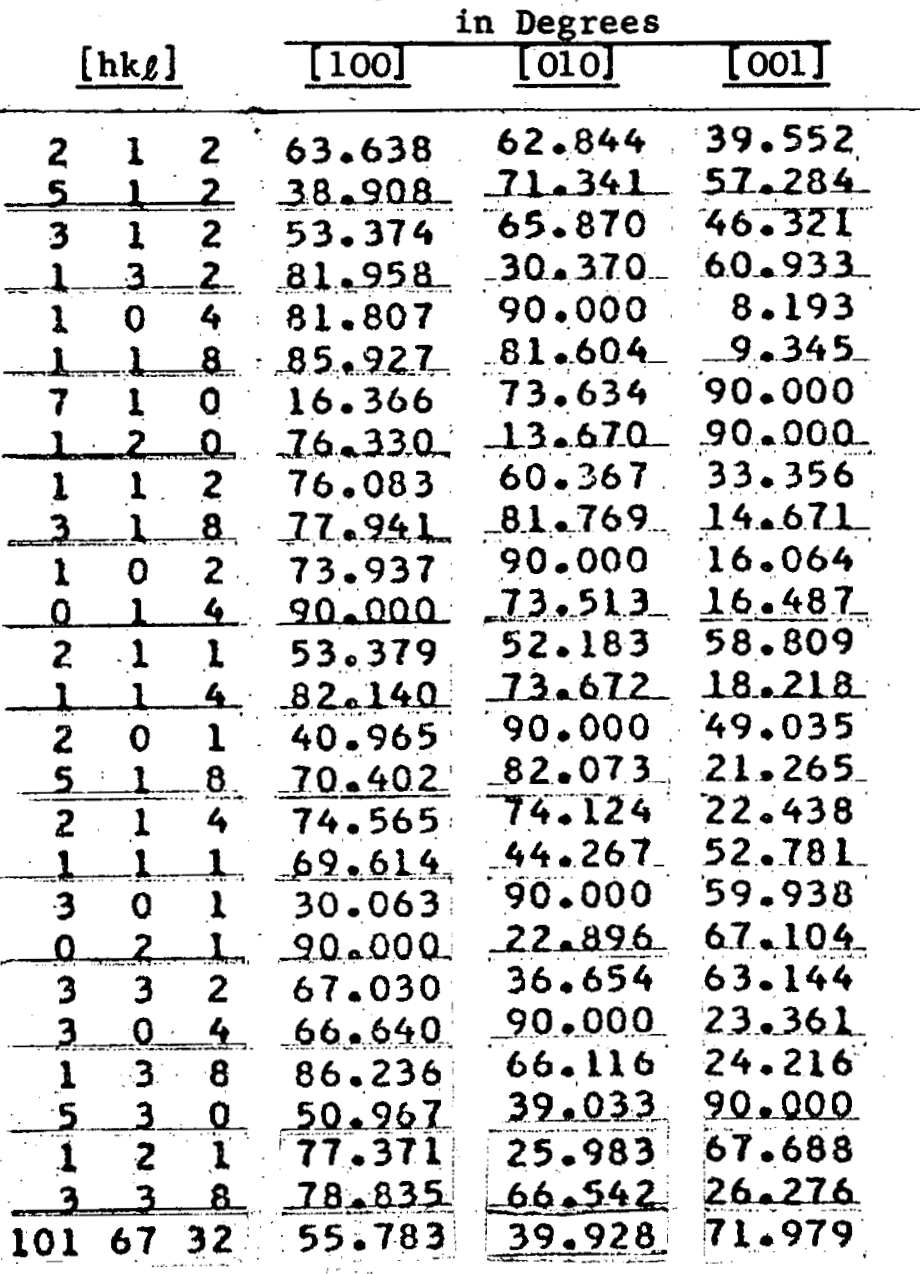


samples except the $1500 \mathrm{ppm} \mathrm{Si}$ alloy were free of $\mathrm{U}_{3} \mathrm{Si}$ precipitates. The 1500 ppm alloy, being quenched from a two-phase region in the beta phase, would be expected to retain its two-phase nature. It should, however, have the maximum possible amount of silicon in solid solution. Samples of the $330 \mathrm{ppm}$ alloy were annealed at $500^{\circ} \mathrm{C}$ or $600^{\circ} \mathrm{C}$ for periods of time ranging from 1 hour up to 300 hours. The $530 \mathrm{ppm}$ alloy and the $1500 \mathrm{ppm}$ alloy were annealed at $600^{\circ} \mathrm{C}$ only. At the end of the annealing treatments, the samples were quenched in water.

\section{Observations}

In order to determine the annealing times which would be favorable for studying the formation of $\mathrm{U}_{3} \mathrm{Si}$ precipitates, samples were annealed at $500^{\circ} \mathrm{C}$ or $600^{\circ} \mathrm{C}$ and then examined in the electron microscope. Equilibrium precipitates of $\mathrm{U}_{3} \mathrm{Si}$ were not observed in any of the samples annealed at $500^{\circ} \mathrm{C}$ for periods of time of up to $300 \mathrm{hrs}$. Figure 3 is typical of photomicrographs of samples which had received the maximum time at temperature. The sample does contain numerous dislocations some of which occur in pairs, which may be an indication of incipient ordering.

An annealing temperature of $500^{\circ} \mathrm{C}$ was therefore viewed as being much too low to achieve precipitation within any treatment duration that would be at all reasonable in the preparation of samples. Thus, all samples annealed after this preliminary experiment were heat treated at $600^{\circ} \mathrm{C}$.

In those samples of the $530 \mathrm{ppm} \mathrm{Si}$ alloy, annealed at $600^{\circ} \mathrm{C}$ for the 1 onger annealing times, $U_{3} S i$ precipitates were observed. For example, in the sample annealed for $100 \mathrm{hrs}$ at $600^{\circ} \mathrm{C}$ there were numerous $U_{3} S i$ precipitates that had grown to over a micron in length and were about 0.1 micron in width. There were other precipitates observable which were in the process of growing at various dislocation sites. The precipitates grew as rod-shaped particles and occurred within grains as well as at grain boundaries.

Figure 4 shows a typical photomicrograph of the sample annealed for $100 \mathrm{hrs}$. Trace analysis revealed that the precipitates were growing at dislocations associated with the slip and twinning planes in uranium. Figure 5 is the diffraction patterns from the central area in $F$ igure 4 . Figure 6 is the analysis of the principal plane traces found in Figure 4 and is based on the diffraction pattern shown in Figure 5. It should be noted that in general it is not possible to identify unambiguously a particular trace umless additional information can be supplied. A first approximation can be obtained by assuming a uniform foil thickness for the area examined. Then by measuring the width of the trace, a foil thickness can be calculated using each assumed plane for each trace. It then should be possible to pick out a set of plane traces which give a fairly uniform foil thickness. Although this has not been done for this particular trace, this procedure has been quite useful in other analyses.

It is also noteworthy in Figure 4 that the precipitate at " $A$ " is forming at a pair of dislocations. This could mean that local ordering may be a stage in 
the formation of the $U_{3} S i$ precipitate.

Examination of the photomicrographs and the electron micrographs of the $530 \mathrm{ppm}$ and $1500 \mathrm{ppm}$ alloys showed increasing precipitation with increasing time at the $600^{\circ} \mathrm{C}$ annealing temperature. Figure 7 contains a series of photomicrographs at 100X. It can be noted that no fine $U_{3} S i$ precipitates are visible in the as quenched $530 \mathrm{ppm} S 1$ specimen although large $\mathrm{U}_{3} \mathrm{Si}$ precipitates are visible in the $1500 \mathrm{ppm}$ alloy. It is evident that the amount of very fine $U_{3} S i$ precipitate increases with increasing annealing time as would be expected. Figure 8 shows an electron micrograph of the $530 \mathrm{ppm}$ alloy that had been annealed at $600^{\circ} \mathrm{C}$ for 7 hours. In the lower half of the picture several $U_{3} S i$ precipitates labeled " $A$ " are visible. In the upper half of the photomicrograph, several very small areas indicated by arrows show what are believed to be Guinier-Preston zones. These zones are approximately $100 \AA$ in diameter and rather sparsely populate this particular allog. The role of Guinier-Preston zones in the formation or nucleation of $\mathrm{U}_{3} \mathrm{Si}$ has not been postulated. The onset of precipitation was also evidenced in the diffraction photographs taken of the specimens. The asquenched samples showed a simple uranium pattern. The patterns for annealed samples became increasingly complex with annealing time up to the point where good $\mathrm{U}_{3} \mathrm{Si}$ patterns also appeared. Further annealing again yielded the simple uranium pattern plus the additional $U_{3} S i$ pattern. The complexity in the pattern revealed itself in the form of rows of related diffraction spots superimposed on the pattern for uranium. Figure 9 shows such a complex pattern. The large spots comprise the main uranium pattern, the smaller spots 1 ined up in rows are anamalous spots due to double diffraction effects from the thin plate-like $\mathrm{U}_{3} \mathrm{Si}$. The orientation of the double diffraction spots, in this particular pattern, suggests that the early stages of precipitation occurred on planes which were approximately $90^{\circ}$ from the (001) plane of uranium. The most probable planes would be those which lie within a few degrees (possibly $10^{\circ}$ ) of the plane of the foil. This relationship is suggested by the fact that the anomalous spots can be indexed in a fashion to show that they are related to each other by a difference of (hk $\boldsymbol{l})$.

Only a limited amount of crystallographic data has been fully interpreted to date in this study. Figure 9 is typical of this type of data. The patterns for uranium (large dots) and $\mathrm{U}_{3} \mathrm{Si}$ (open circles) can be graphically manipulated to reveal orientation relationship between the precipitate and the matrix. Figure 10 shows the relationships obtained from the patterns in Figure 9. The following orientation relationships, as well as others, can be seen on the stereographic projection:

$$
\begin{aligned}
& (100)_{\mathrm{m}} \|(101)_{\mathrm{p}} \\
& (011)_{\mathrm{m}} \sim \|(010)_{\mathrm{p}} \\
& (001)_{\mathrm{m}} \sim \|(122)_{\mathrm{p}},
\end{aligned}
$$

where $m=$ matrix and $p=$ precipitate. 
Due to the fact that the precipitate has been observed to form on slip or twinning planes, many other orientation relationships are possible. In all cases where anomalous spots were observed the plane of the foil was within $10^{\circ}$ of a twinning plane.

The crystallographic relationships quoted above should be regarded as strictly arbitrary since it was necessary to assign arbitrary plus or minus signs to the hke's of the diffraction spots when indexing the pattern. However, by performing tilting experiments, it should be possible to index in three dimensions and thereby eliminate any doubt as to the correct sign of the Miller indices for any spot. If Kikuchi patterns were easier to obtain, this would also be a means of eliminating such uncertainty.

Figure 11 shows three diffraction patterns as well as a polar diagram of the position of the perpendicular to the plane of the sample relative to the electron beam. Patterns 1 and 2 both contain a row of spots labeled (021), (042), and (063) but no others which index alike. In patterns 2 and 3 , the common row is the one containing the $(200)$ and (400) reflections. Patterns 1 and 3 are tilted $5^{\circ}$ in relation to pattern 2 but in mutually perpandicular directions as shown on the polar diagram. The patterns represent three planar sections through an array of points in space, and the common rows of spots are along the line of intersection of the two planes involved. In this way an unambiguous determination of spacial relationships can be made between the matrix and the precipitate since the orientation of the specimen can be uniquely determined.

\section{EXAMINATION OF A URANIUM-ALUMINUM ALLOY BY TRANSMISSION ELECTRON MICROSCOPY}

At the request of $R$. Schierding and $L$. Fergason, who were working on uranium phase diagrams, a sample of a uranium alloy was examined by transmission electron microscopy. The sample contained $2000 \mathrm{ppm}$ aluminum and had been annealed for 4 months at $550^{\circ} \mathrm{C}$ and water quenched. The sample when examined by light microscopy showed a very fine second-phase material whose size was about at the resolution limit of optical microscopy and not as large size agglomerates as expected.

Normal sample preparation procedures were followed for the preparation of a thin foil from this material. When examined the foil revealed rod or needle shaped particles with a diameter of about $1000 \AA$ to $1500 \AA$ and were from $4000 \AA$ to $10,000 \AA$ in length. An electron diffraction pattern from an area containing one of these particules was obtained. The pattern contained both the uranium pattern and the precipitate pattern. Figure 12 shows the two patterns with the measured d spacing for the precipitate pattern shown beside the spots.

The diffraction spots cannot be indexed as $\mathrm{UAl}_{2}$ but could be indexed as $\mathrm{UAl}_{3}$ as shown in Table VIII. However, this was not consistent with the composition of the sample. Further study revealed that the pattern could be indexed as $\mathrm{U}_{3} \mathrm{Si}$.with an expanded lattice, having $\mathrm{a}_{0}$ expanded from $6.029 \AA$ to $6.080 \AA$ 。 This is more consistent with the sample composition since it did contain a 
small amount of silicon. Other samples from this metal and one prepared especially as a standard for $\mathrm{VAl}_{2}$ gave patterns which indexed perfectly for $\mathrm{UAl}_{2}$. It is easy to see from this experience that some common sense is needed along with the facility to index patterns.

\section{Table VIII}

Comparative Indexing of the Diffraction

Pattern in Figure 12

\begin{tabular}{|c|c|c|c|c|}
\hline \multirow[b]{2}{*}{ d measured } & \multicolumn{2}{|c|}{$\mathrm{UAl}_{3} \mathrm{a}_{0}=4.287 \AA$} & \multicolumn{2}{|c|}{$\begin{array}{l}\mathrm{U}_{3} \mathrm{Si} \text { with an expanded lattice } \\
\mathrm{a}_{0}=6.080 \AA\end{array}$} \\
\hline & hke & d & hke & $\mathrm{d}$ \\
\hline 4.298 & 100 & 4.287 & 110 & 4.299 \\
\hline 2.149 & 200 & 2.143 & 220 & 2.150 \\
\hline 3.042 & 110 & 3.031 & 200 & 3.040 \\
\hline 1.919 & 210 & 1.917 & 310 & 1.923 \\
\hline 1.529 & 220 & 1.516 & 400 & 1.520 \\
\hline
\end{tabular}

STUDIES OF FLON IN URANIUM BY TRANSMISSION ELECTRON MICROSCOPY

\section{Foil Tensile Samples}

Attempts have been made to produce tensile specimens suitable for use in the transmission electron microscope. The desired configuration would be an hourglass shape where the deformation could be restricted to the reduced cross section. So far, such attempts have been unsuccessful. The added polishing necessary to produce this configuration has resulted in highly unsatisfactory thin sections at the constriction: This area of the specimen has become highiy oxidized, and the removal of this oxide without destroying the specimen has been the major problem.

Mechanisms. of Flow in Standard Hot Tensile Tests

Thin sections of full-sized tensile specimens pulled at room temperature, $300^{\circ} \mathrm{C}$ and $500^{\circ} \mathrm{C}$ have been examined. The samples came from centrifugally cast cores with the room temperature specimen analyzing $190 \mathrm{ppm} \mathrm{Fe;} 160 \mathrm{ppm} \mathrm{Si}, 530 \mathrm{ppm} \mathrm{C}$, and $1.7 \mathrm{ppm} \mathrm{Hz}$. The similar $300^{\circ} \mathrm{C}$ and $500^{\circ} \mathrm{C}$ test specimens analyzed $174 \mathrm{ppm} \mathrm{Fe}$, $320 \mathrm{ppm} \mathrm{Si}, 1200 \mathrm{ppm} \cdot \mathrm{C}$, and $18 \mathrm{ppm} \mathrm{H}$.

The room temperature specimen, Figure 13 was very heavily twinned and had a very high concentration of dislocations. In spite of the small (2000 $\AA$ wide) 
poorly formed twins, it was possible to identify the twin system operating through diffraction and trace analysis. There were four systems that would fit the trace analysis:

\begin{tabular}{|c|c|c|c|c|c|}
\hline System & $\mathbf{k}_{1}$ & $\mathrm{k}_{2}$ & $\eta_{2}$ & $\eta_{2}$ & s \\
\hline 1 & $(\ddot{\infty} 1)$ & $(8 \overrightarrow{2} 1)$ & {$[100]$} & [132] & 0.286 \\
\hline 2 & $(4 \overline{2} 1)$ & $(\bar{O} 1)$ & {$[\overline{2} \overline{3} 2]$} & {$[100]$} & 0.566 \\
\hline 3 & $\left(\bar{\infty}_{1}\right)$ & $(8 \overline{6} 3)$ & {$[100]$} & {$[\overline{332}]$} & 0.849 \\
\hline 4 & $(\overline{8}, \overline{9} \overline{6}, 37)$ & $(001)$ & [138] & [130] & 0.900 \\
\hline
\end{tabular}

The above are illustrated in Figure 14. The notation $k_{1}$ refers to the twin plane, $K_{2}$ the reciprocal twin plane, $\eta_{1}$ the twinning direction, $\eta_{2}$ the reciprocal twinning direction, and $S$ is the amount of sheer. Thus $K_{11}$ is the the $k_{1}$ plane for System $1, K_{21}$ is the reciprocal twin plane for System 1 , and $\mathbf{R}_{12}$ is the twin plane for twin System 2. $P_{1}$ and $P_{2}$ represent the normals to the two crystal systems in the diffraction pattern and since the section examined was a transverse section, they represent the tensile axis.

The decision as to which twin system was operating is determined by a process of elimination. System No. 4 can be excluded since $\eta_{1}$, the twinning or sheer direction [138], is over $70^{\circ}$ from the tensile axis and the amount of sheer is very high, 0.900 . A decision between Systems 1 and 3 must be made on the basis of the much greater amount of sheer needed for System No. 3, since the other factors are about equal. Systems 1 and 2 are almost conjugate systems. The $\eta_{11}$ is $35^{\circ}$ from $P_{1}$ while $\eta_{12}$ is on $1 y 9^{\circ}$ from $P_{2}$, but $s_{1}$ is 0.286 while $s_{2}$ is 0.566 . The twin System No. 2 has the greatest resolved sheer but also requires more sheer to operate. The choice is between-Systems 1 and 2 and in all probability both systems were operating.

The $300^{\circ} \mathrm{C}$ specimen, Figure 15 appeared to be twinned also, but the twins were up to $1 \mu$ in width or five times the size of those in the room temperature specimen. The twin boundaries were very straight; the dislocation density was very much lower and well separated from the boundaries. Trace and diffraction analyses have not been completed on this sample, but it appears to be at least partially polygonized due to the slow cooling in the furnace after the tensile test.

Transverse sections from the $500^{\circ} \mathrm{C}$ tensile specimen were examined. The photograph, Figure 16, shows what appear to be twins that range in width from 0.1 micron to 0.3 micron and have slightly curved boundaries. The diffraction pattern, Figure: 17. from a selected area, shows a series of spot patterns about an axis normal ..... to the plane on the foil. Each spot pattern is rotated only a few degrees from the other spots. Analysis of the bands of dislocation networks shows them to be on (172), (111), and (112) planes. A calculation of foil thickness from a 
narrow dislocation band and a wide dislocation band shows the foil to be $154 \AA$ and $166 \AA$ thick, respectively, at these bands. Since the locations were within $0.5 \mu$ of each other, it appears that the data on the planes are mutually consistent.

The fact that essentially one diffraction pattern was obtained and that the dislocation networks are on known slip planes suggests that the deformation at $500^{\circ} \mathrm{C}$ was occurring by slip. If twinning had occurred, it would have produced two or more diffraction patterns and the boundaries between the twins would have been coherent and showed stalking fault contrast rather than dislocation networks. This condition is quite apparent when the grain boundaries in Figure 13 and Figure 16 are carefully examined.

\section{Conclusions}

Even though the investigation is not complete, it has been demonstrated that a post-mortem of a tensile specimen by thin-foil analysis can be used to study the deformation mechanisms occurring in uranium. Thin-foil analyses, coupled with a knowledge of second-phase morphology, should permit an assessment of deformation mechanisms and metal stability in relation to alloying and heat trea tment. 


\section{References}

1. Buckley, S. No, Irradiation Growth, UKAEA Report AERE-R 3674 (March 1961).

2. Bridge, J. R.; Schwartz, C. M., Vaughan, D. A , "X-Ray Diffraction Determination of the Coefficients of Expanston of Alphamuranium. ${ }^{\circ 1}$ Journal of Metals (Transactions of the AIME) Vol。 8 , No.10, sect. 2 (October 1956), pp: 1282-5.

3. Barrett, C. S , Structure of Meta1s, McGraw 1952.

4. Zachariasen, W. H., "Crystal Chemical Studies of the $5 \mathrm{f}$-Series of Elements" VIII. Crystal Structure Studies of Uranium Silicides and CeSi $i_{2}$ and PuSia." Acta Crystallographica Vol.2 (1949), pp.94\%9.

5. Mueller, M。 Ho, and Knott, $H_{0} . W_{0}$, Argonne National Laboratory, Personal Communication.

6. Crocker, A. G., "The Crystallography of Deformation Twinning in Alpha. Uranium." Journal of Nuclear Materials Vol。16 (1965), pp。306-326. 


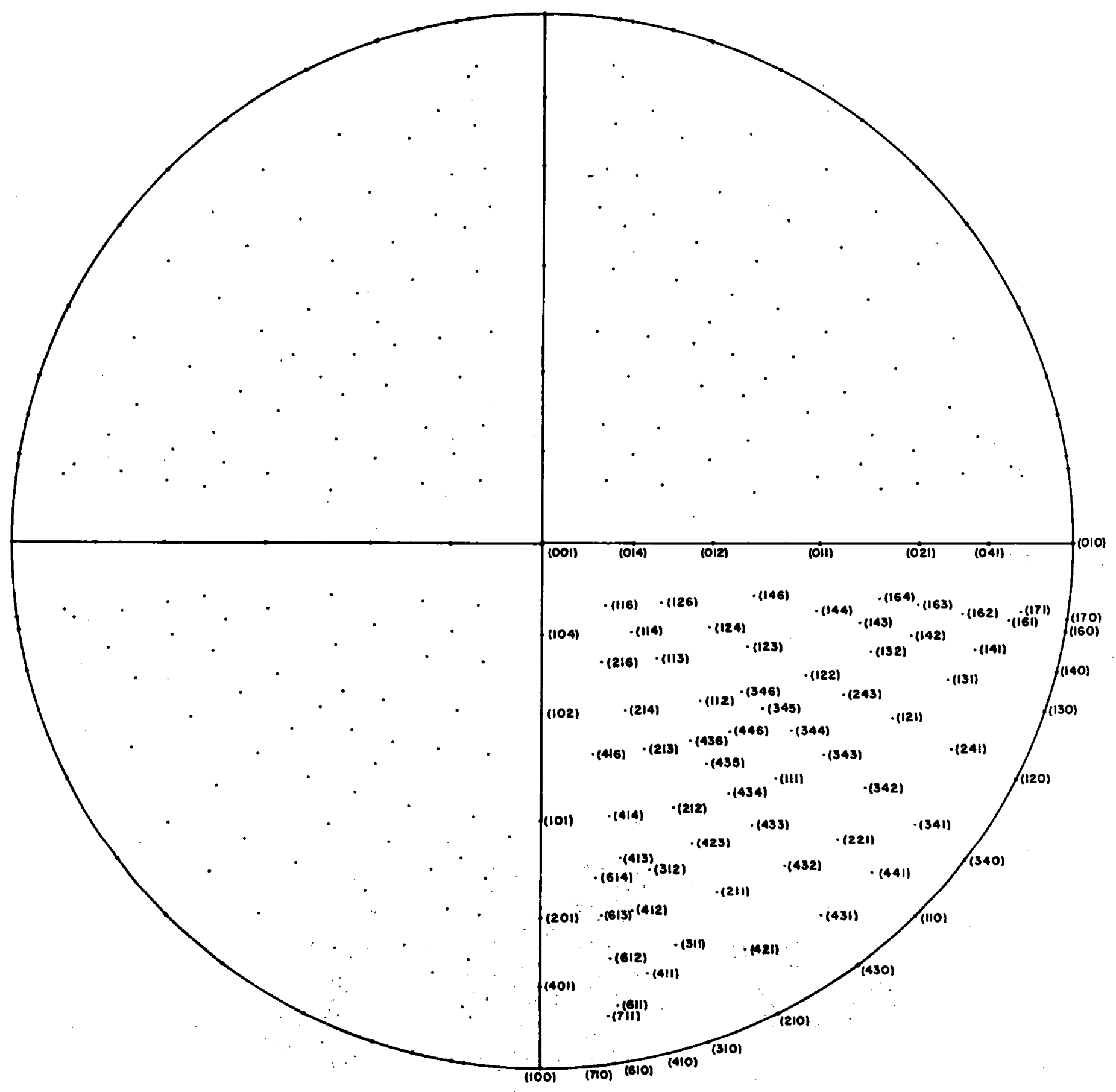

Figure 1. Standard (001) Projection for $\mathrm{U}_{3} \mathrm{Si}$.

Prepared by H. C. Kloepper, Jr.,

Mallinckrodt Chemical Works, Uranium Division,

St. Charles, Missouri 


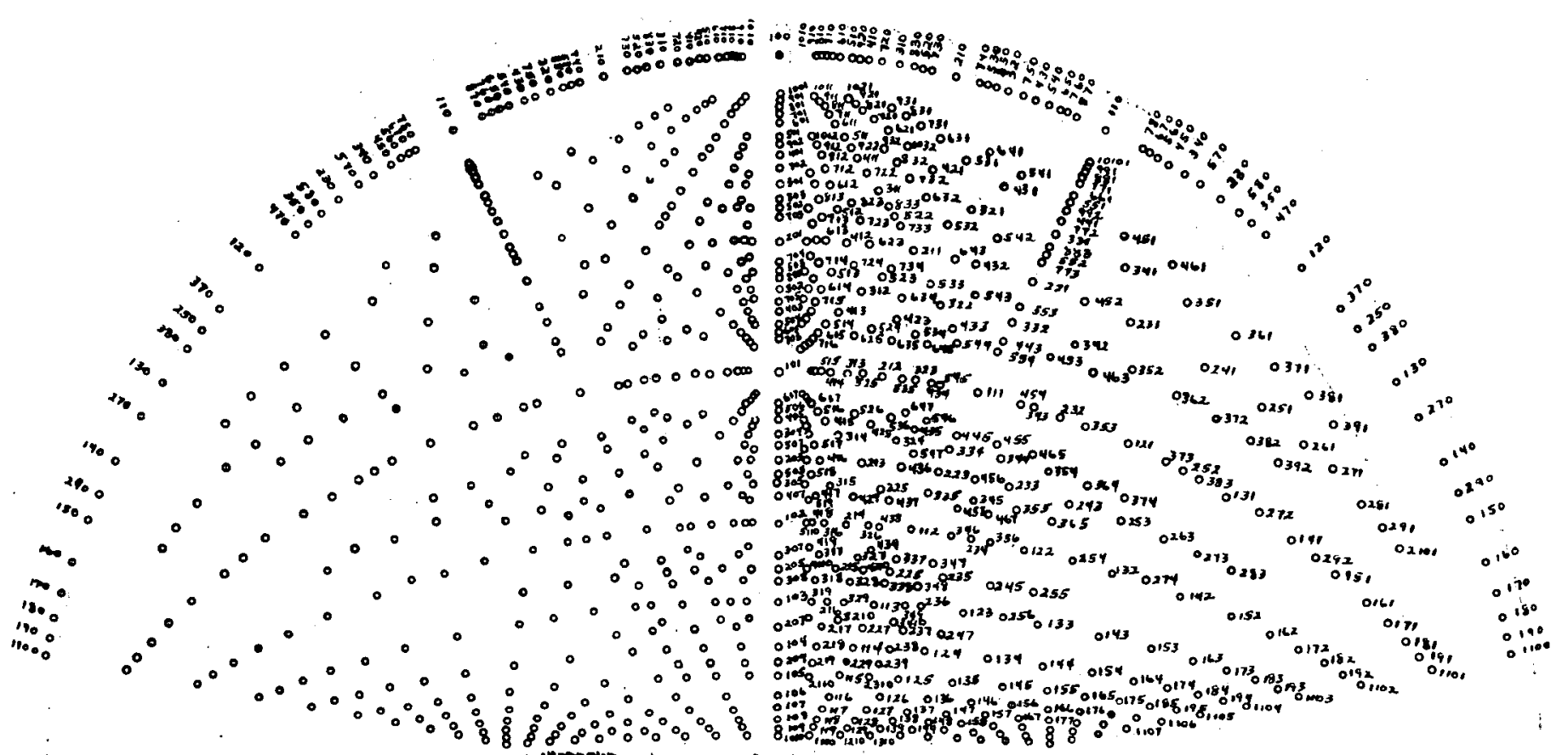

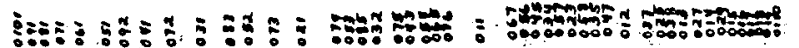

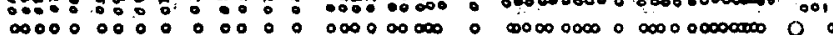

$0000000000000000000000000000000000000000 \quad 0010$

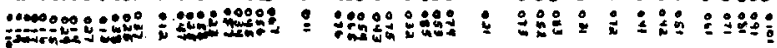

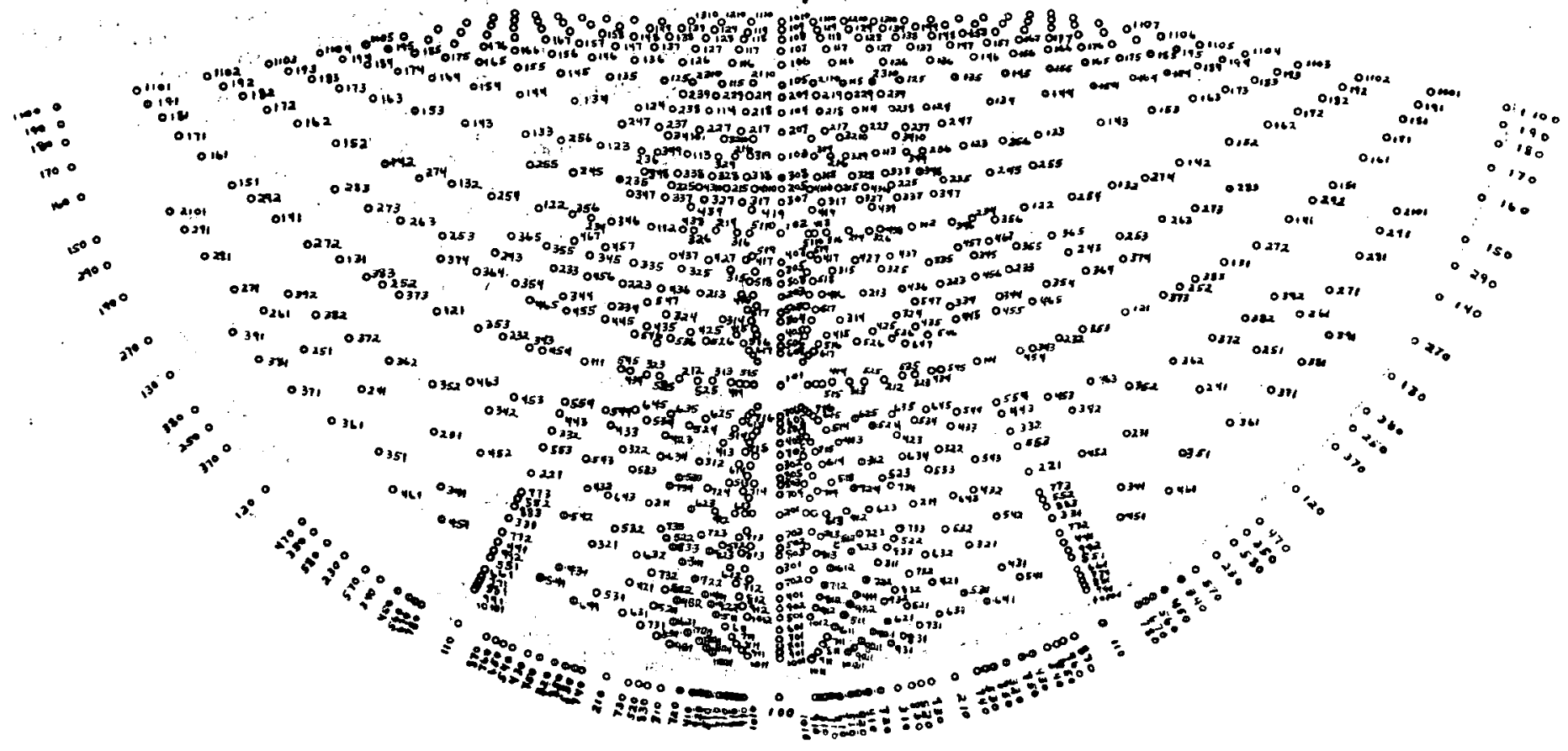

Figure 2. Standard (100) Projection for Alpha-Uranium.

Prepared by M. H. Mueller and H. W. Knott, Argonne National Laboratory, Lemont, Illinois. 


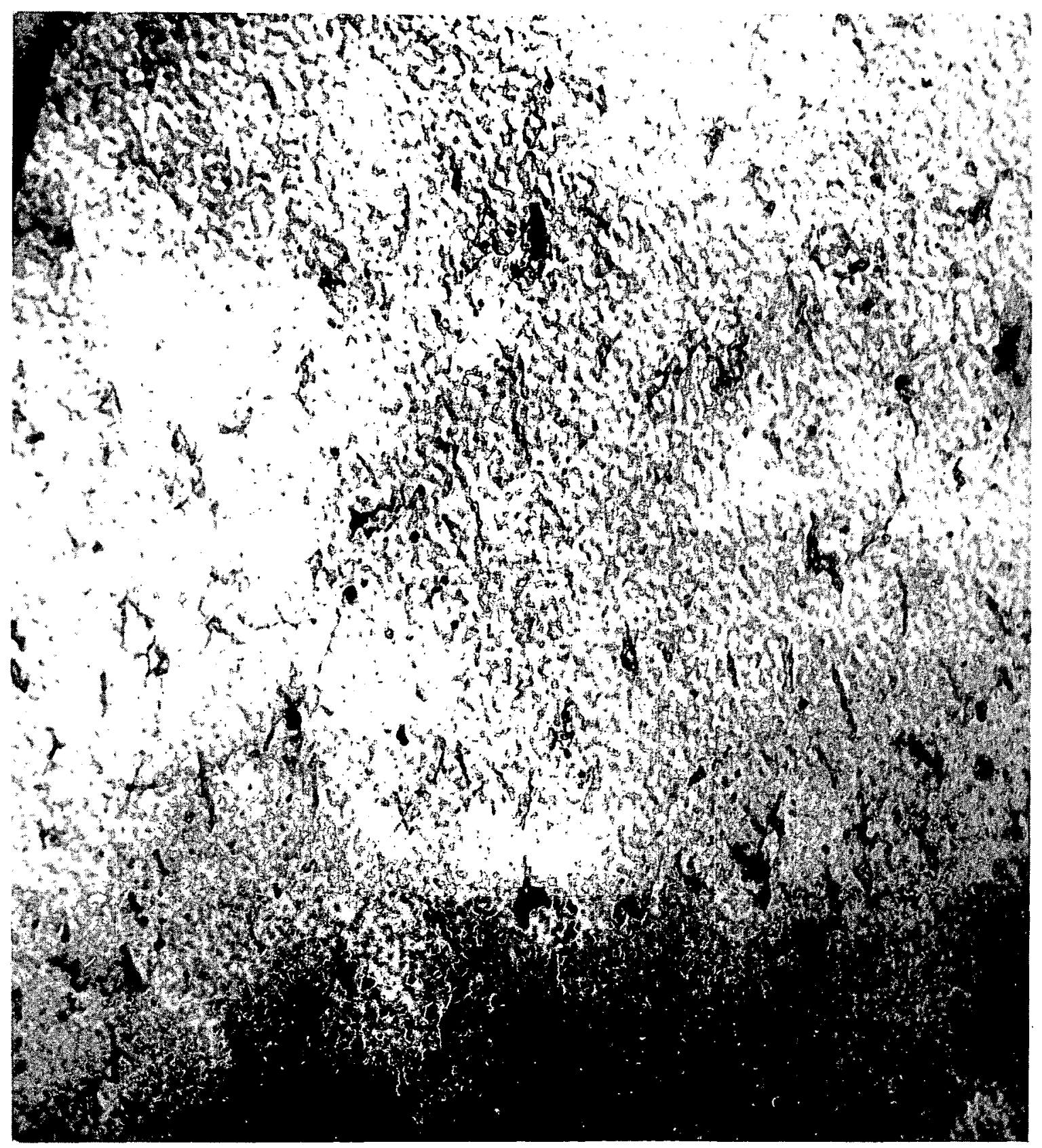

Figure 3. Uranium with $330 \mathrm{PPM} \mathrm{Si}$, Solution-Treated 24 Hours at $700^{\circ} \mathrm{C}$ and Water-Quenched, Annealed 306 Hours at $500^{\circ} \mathrm{C}$ and Water-Quenched. (Plate $7 ; 54,600 \mathrm{X}$ ). 


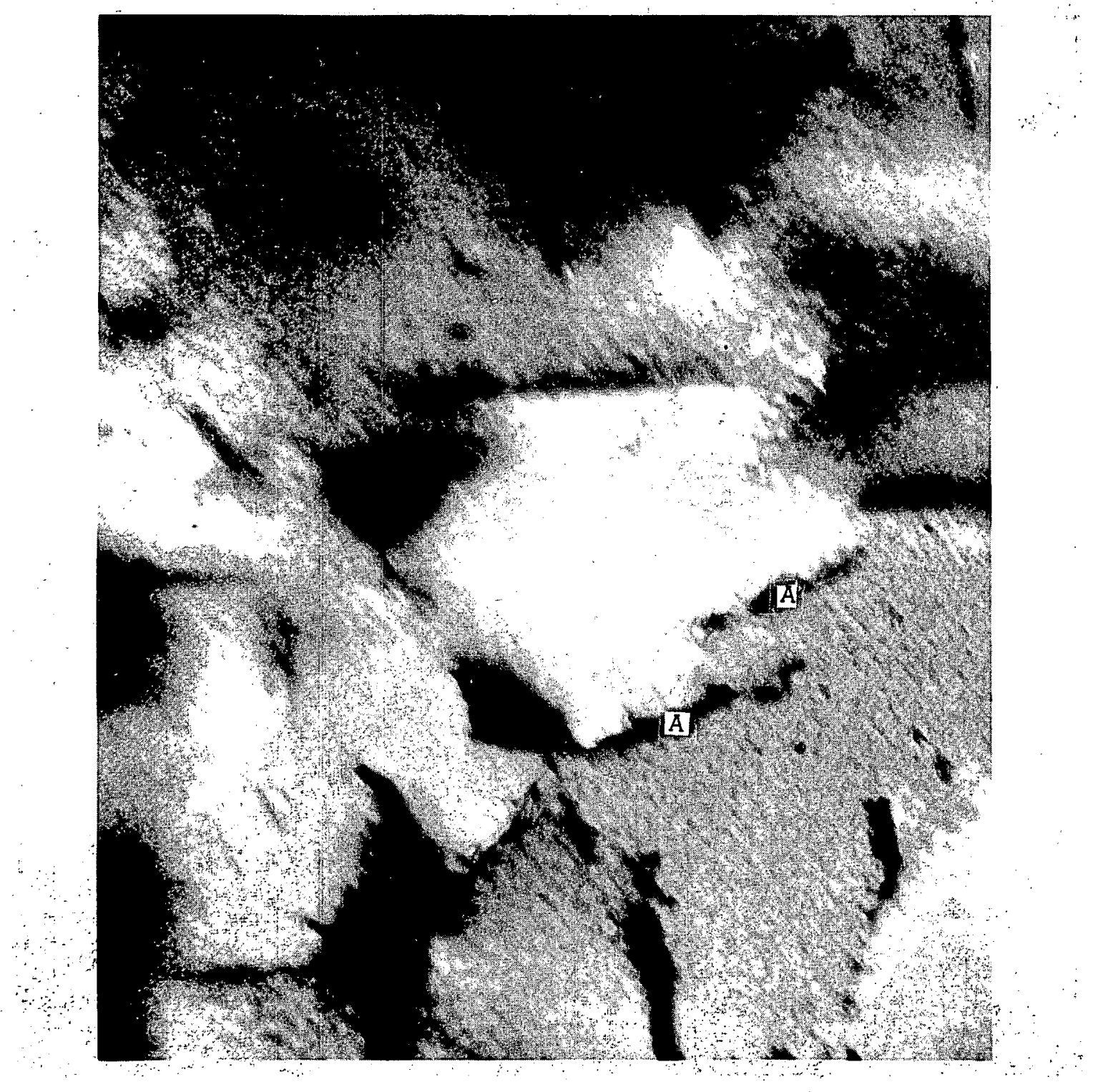

Figure 4. Uranium with 530 PPM Si, Solution-Treated 48 Hours at $750^{\circ} \mathrm{C}$ and Water-Quenched, Annealed 100 Hours at $600^{\circ} \mathrm{C}$ and Water-Quenched. The precipitates $\left(A^{\prime} \mathrm{s}\right.$.) are forming on a pair of dislocations. (Plate $522 ; 51,600 \mathrm{X}$ ) 
O (i门̄)

$$
O(\overline{1} 3.2)
$$

$$
0(0 \overline{2} \overline{3})
$$

$$
O(T, T)
$$

$$
\text { (0) }\left(\begin{array}{lll}
0 & 0 & 0
\end{array}\right)
$$

\section{$O_{(1 \overline{3} \overline{2})}$}

$$
\begin{array}{cc}
0(1 \overline{1}) & 0(023) \\
0(2 \overline{2} 2) & 0(114) \\
\vdots & 0(205) \\
0(3 \overline{3} 3) &
\end{array}
$$

Figure 5. Diffraction Pattern from the Central Area in Figure 4. 


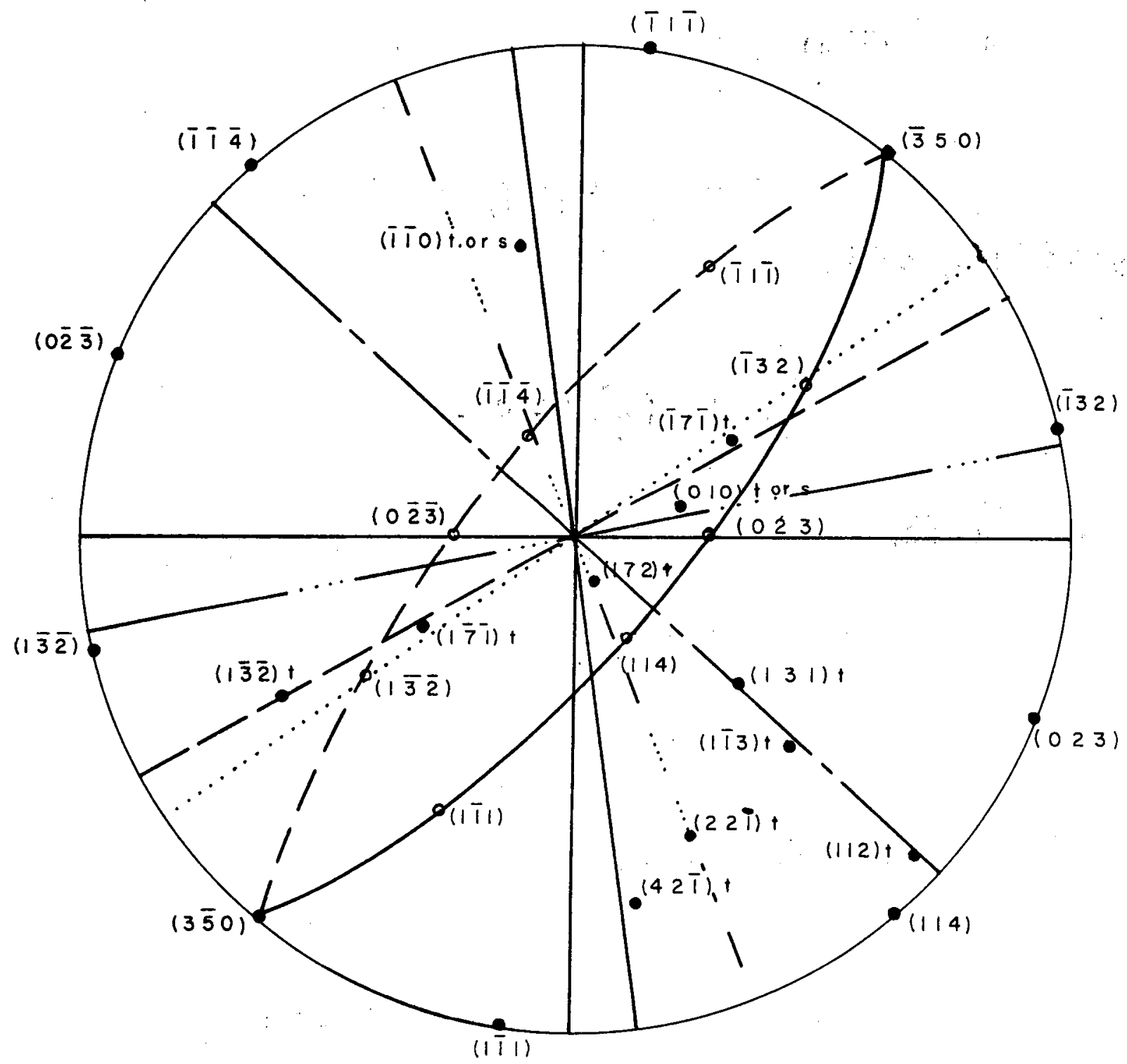

Figure 6. Trace Analysis of Five of the Principal Precipitation Planes in Figure 4. A $10^{\circ}$ clockwise rotation of the above figure will bring the plane normals (dotted or dashed lines) into coincidence with the plane traces in Figure 4. The most probable planes (solid dots) giving rise to these traces lie along the plane normals in the above figure.

$$
(t=t w i n ; s=s l i p)
$$



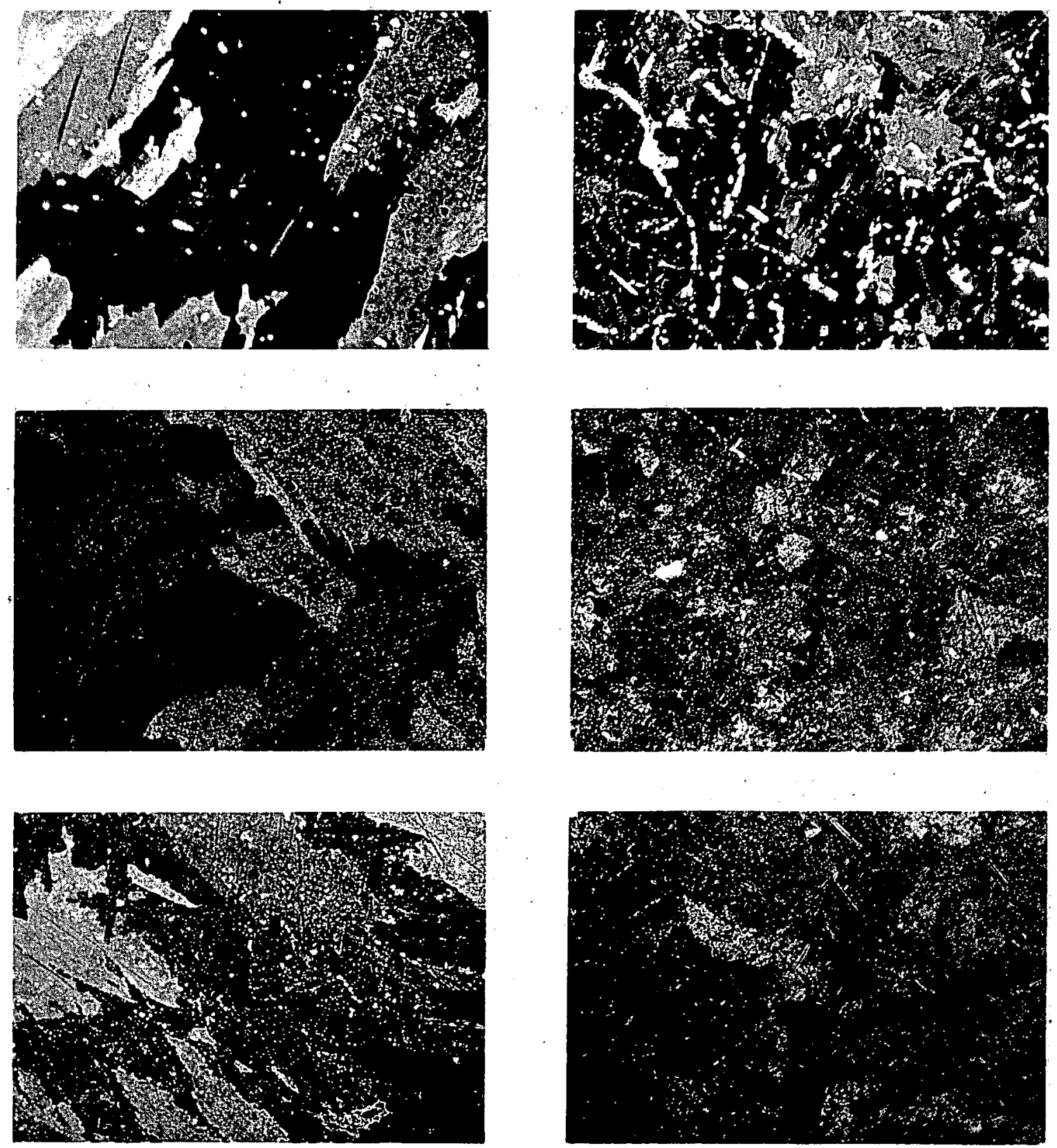

Figure 7. Polarized Light Photomicrographs (100X) of Solution Heat-Treated Alloys of Uranium Containing 530 PPM Silicon (Left Column) and 1500 PPM Silicon (Right Column). The precipitation resulting from annealing at $600^{\circ} \mathrm{C}$ for 0,7 , and 30 hours, respectively, is shown (top to bottom). 


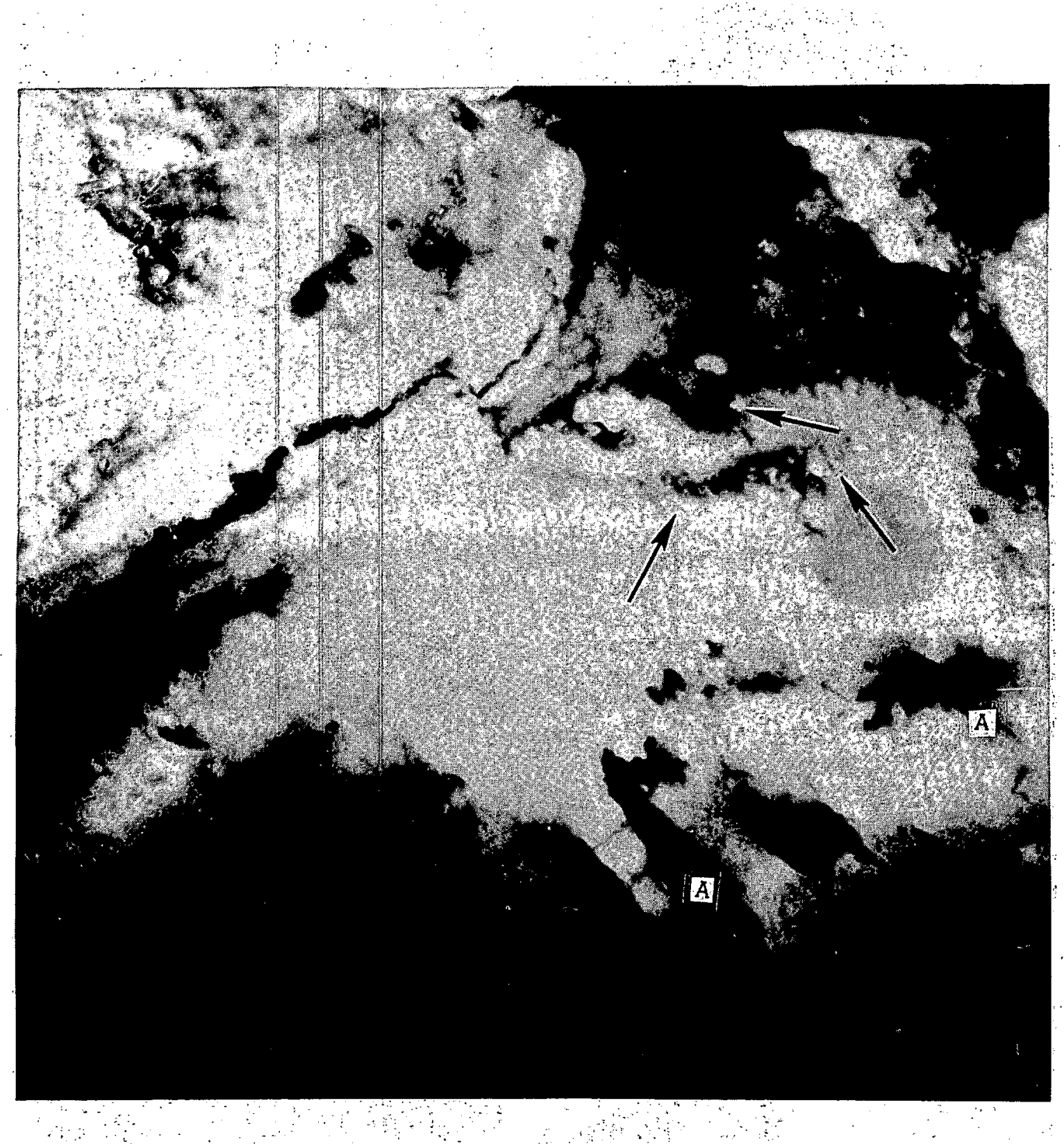

Figure 8. Uranium with $530 \mathrm{PPM} \mathrm{Si}$, Solution-Treated and Aged 7 Hours at $600^{\circ} \mathrm{C}$. Note the $U_{3} S i$ precipitates ( $A^{\prime} s$ ) and the Guinier-Preston zones (arrows). 


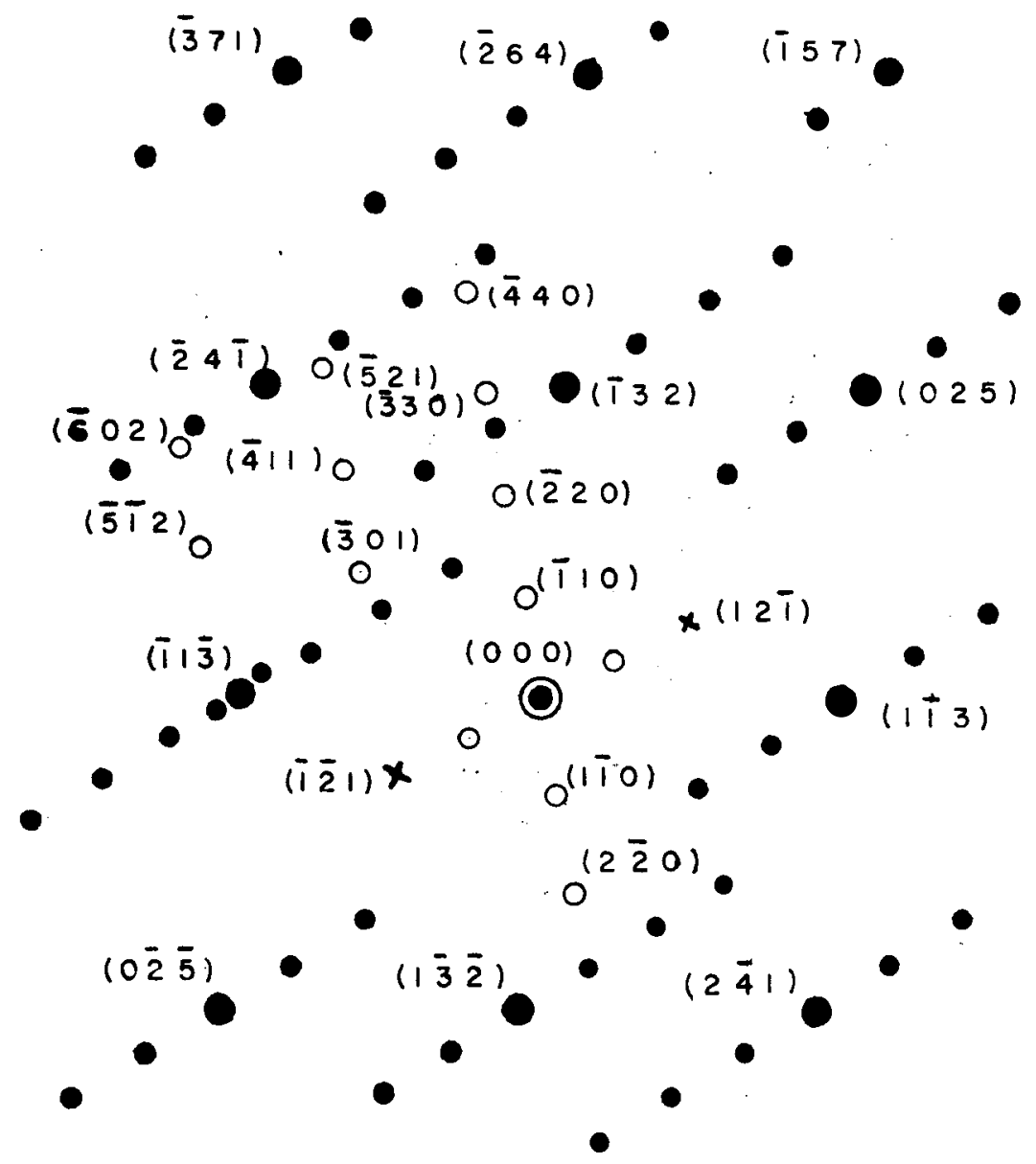

Figure 9. Scale Drawing of the Diffraction Pattern from an Alloy of Uranium Containing 1500 PPM Si, solution-treated and annealed 7 hours at $600^{\circ} \mathrm{C}$. The open circles are $\mathrm{U}_{3} \mathrm{Si}$; the large solid dots are uranium; the small solid dots are anomalous uranium spots obtained by doubled diffraction. 


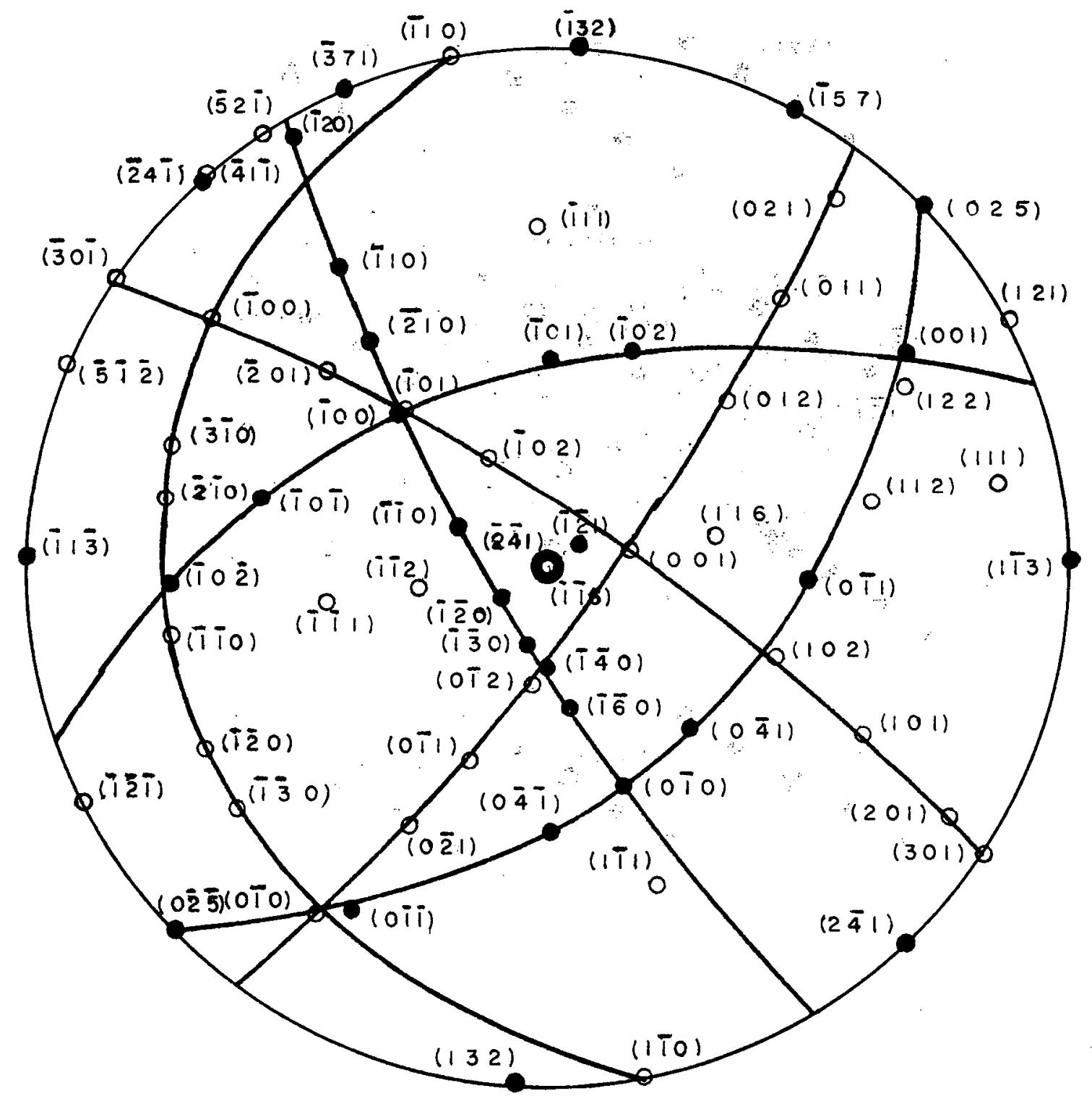

Figure 10. Stereographic Projection of the Data Obtained from the Di ffraction Pattern Shown in Figure 9. The open circles are the location of the poles from the $\mathrm{U}_{3} \mathrm{Si}$ precipitate. The solid circles represent the poles from the uranium matrix. 


\section{(․․․․}

(333) (3일 (311) $\quad$ (330)

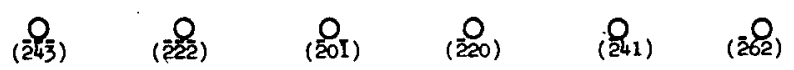

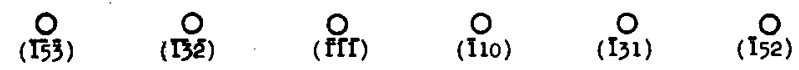

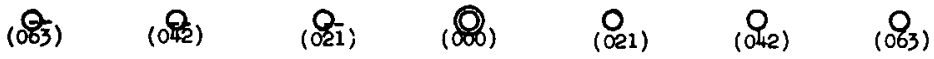

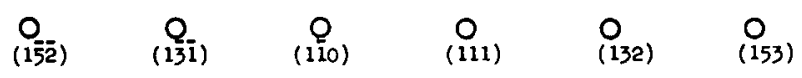

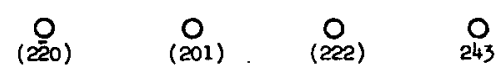

$\stackrel{\circ}{(\text { (3i1) }} \stackrel{\circ}{(312)}$

Pattern 1

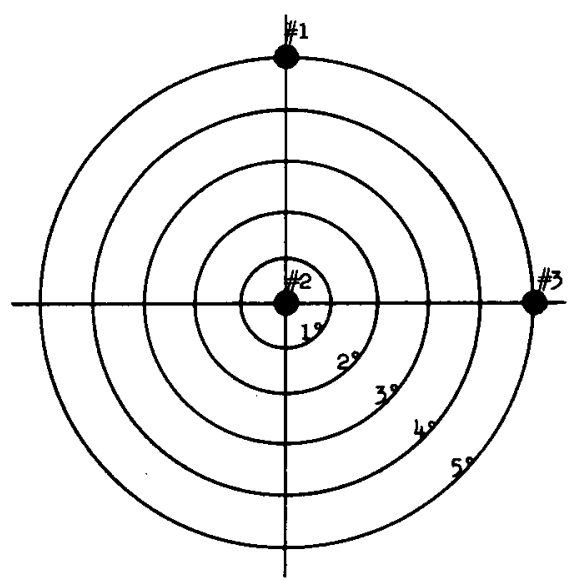

$\underset{(531)}{\mathrm{O}}$

$\stackrel{0}{\circ} \quad \stackrel{0}{(44 i i)} \quad \stackrel{0}{(42 i)}$

$\stackrel{\circ}{(400)}$

(331)

$\stackrel{\circ}{(331)}$

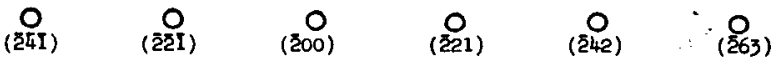

$\stackrel{0}{(200)}$

$(\stackrel{\circ}{(i \bar{i})}$

$\stackrel{\circ}{(i 31)}$

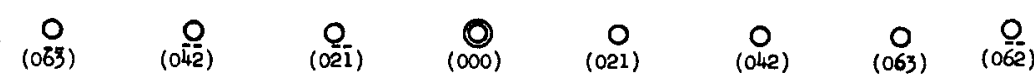

$\underset{(000)}{(0)}$

(13i)

$\underset{2 \overline{2} \overline{1}}{0} \quad \underset{(200)}{0} \underset{(221)}{0} \quad \underset{(242)}{0}$

Pattern 2 $\underset{(131)}{0}$

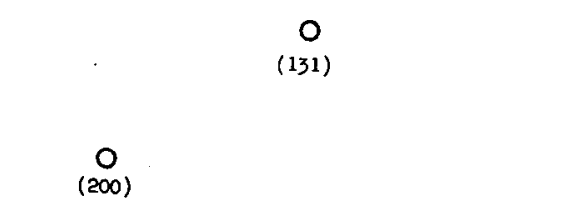

Pattern 3

Figure 11. Diffraction Patterns from the Same Area of a Foil. The foil was perpendicular to the beam in Pattern 2. In Patterns 1 and 3, the foil was tilted so that the direction perpendicular to the foil was in position indicated in the polar diagram. 


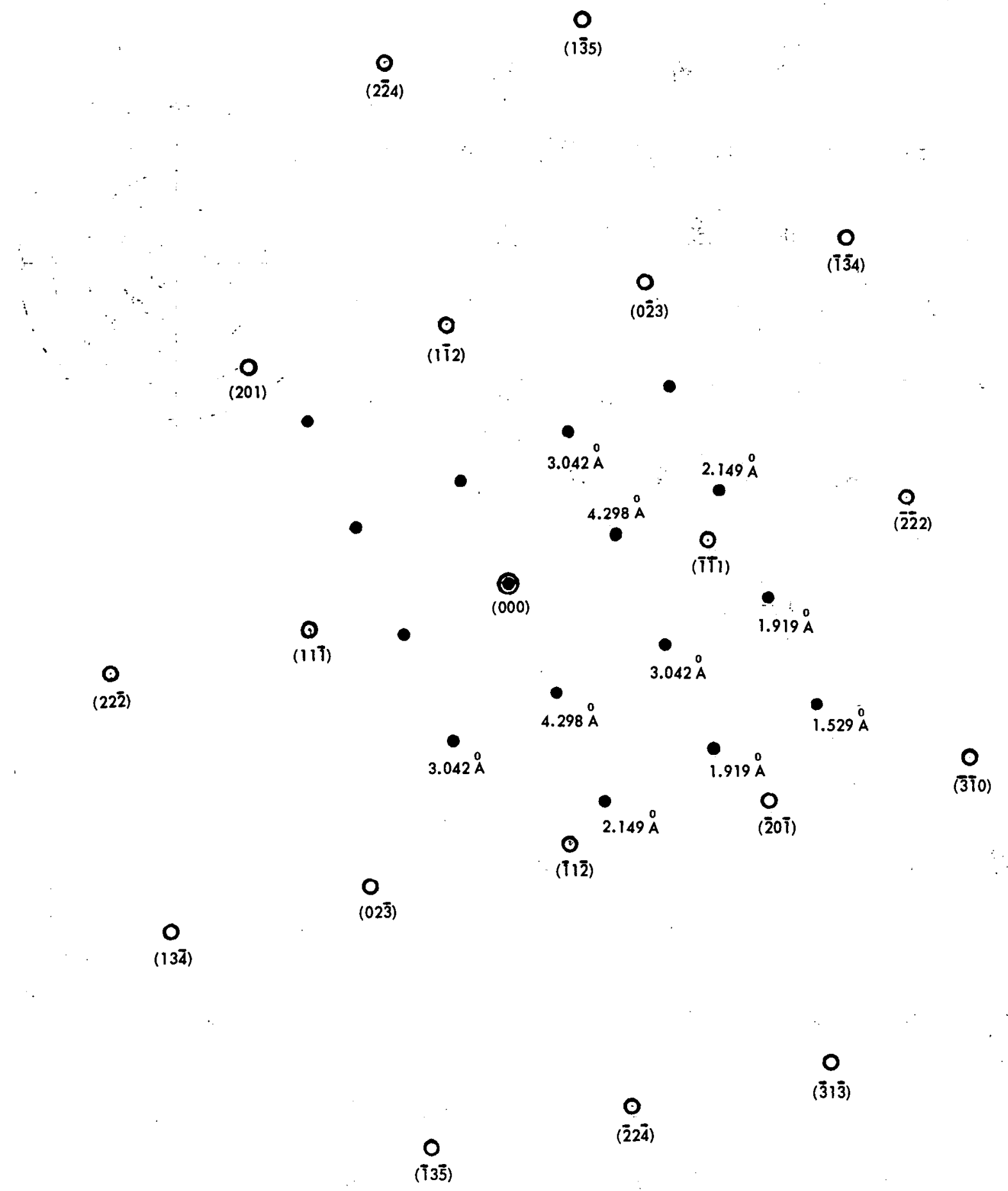

Figure 12. Diffraction Pattern from a 2000-PPM Al Alloy of Uranium. The open circles are the uranium pattern. The dots with their " $d$ " spacings beside them are from a precipitate particle. The pattern appears to be $U A l_{3}$, but is not consistent with the composition. A better solution is $\mathrm{U}_{3} \mathrm{~S}$ "with an expanded lattice. 


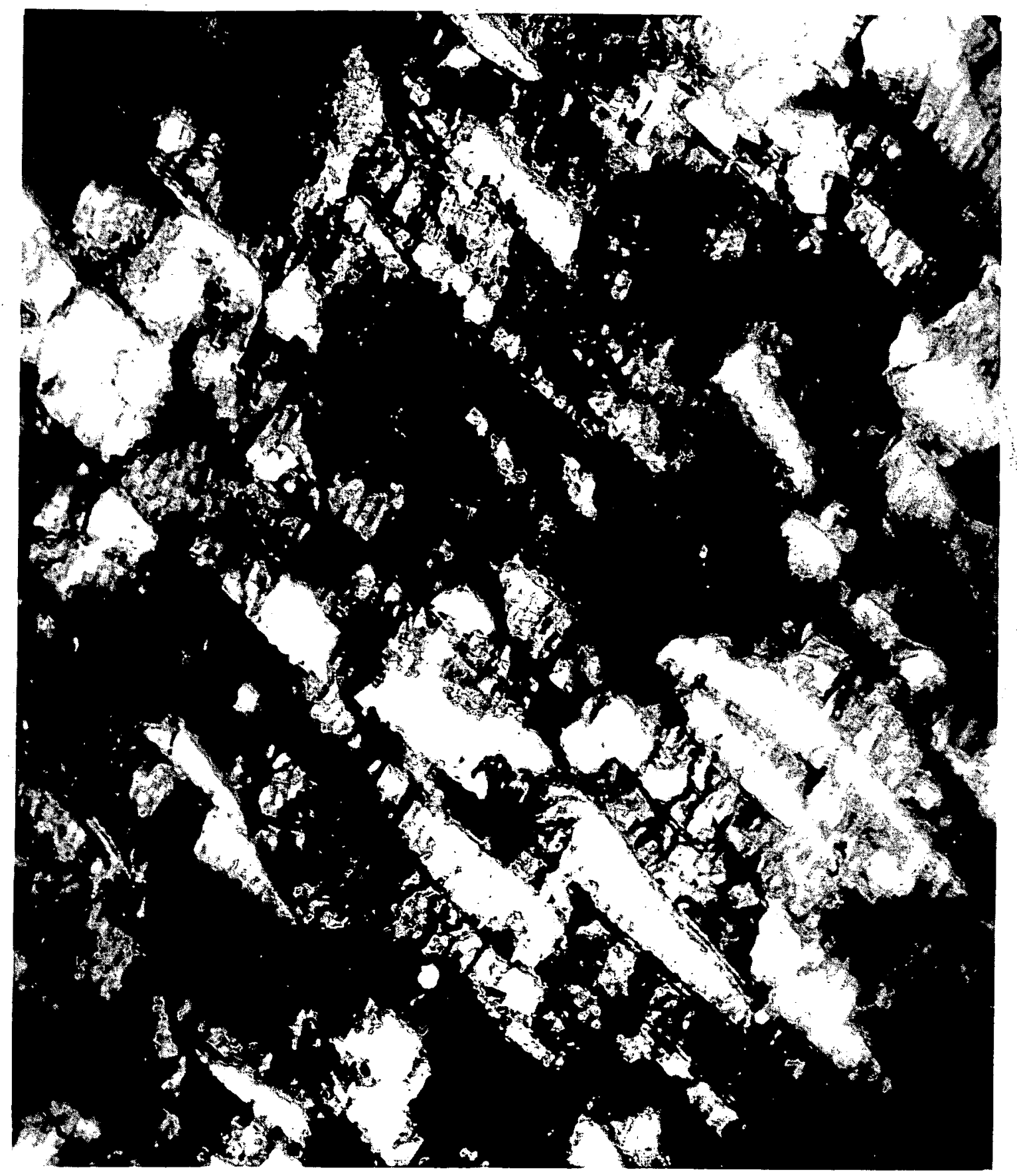

Figure 13. A Transmission Electron Micrograph of the Uranium Tensile Specimen at Room Temperature, Showing the Transverse Section Near the Break. Note the coherent boundaries (parallel lines) between twins. (Plate $855 ; 86,100 \mathrm{X}$ ) 


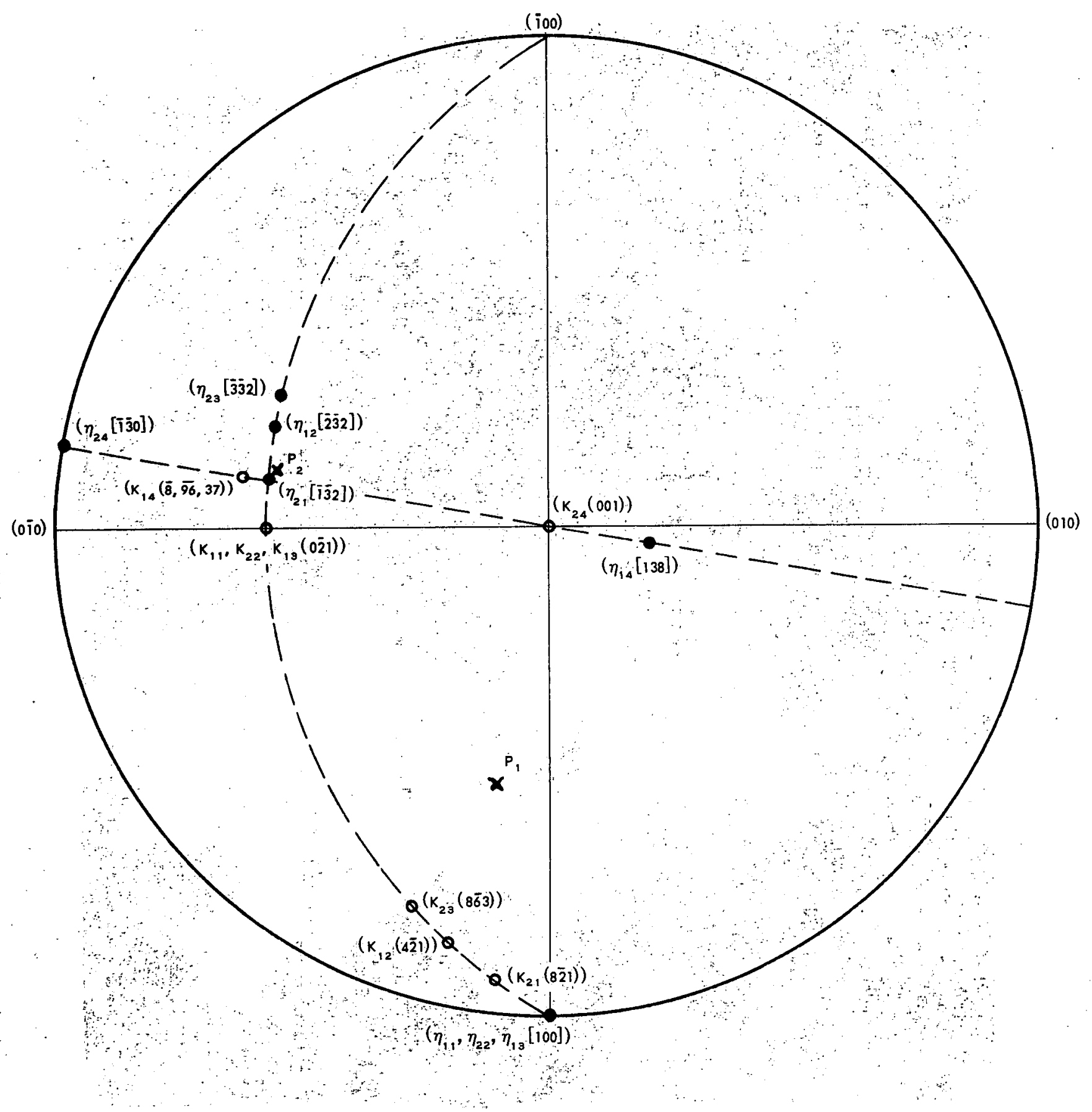

Figure 14. Possible Twin Systems in the Room-Temperature Tensile Specimen Shown on an (001) Projection for Uranium. Note that for any twin system $\mathrm{K}_{1}, \mathrm{~K}_{2}, \eta_{1}$, and $\eta_{2}$ lie in the same plane. 


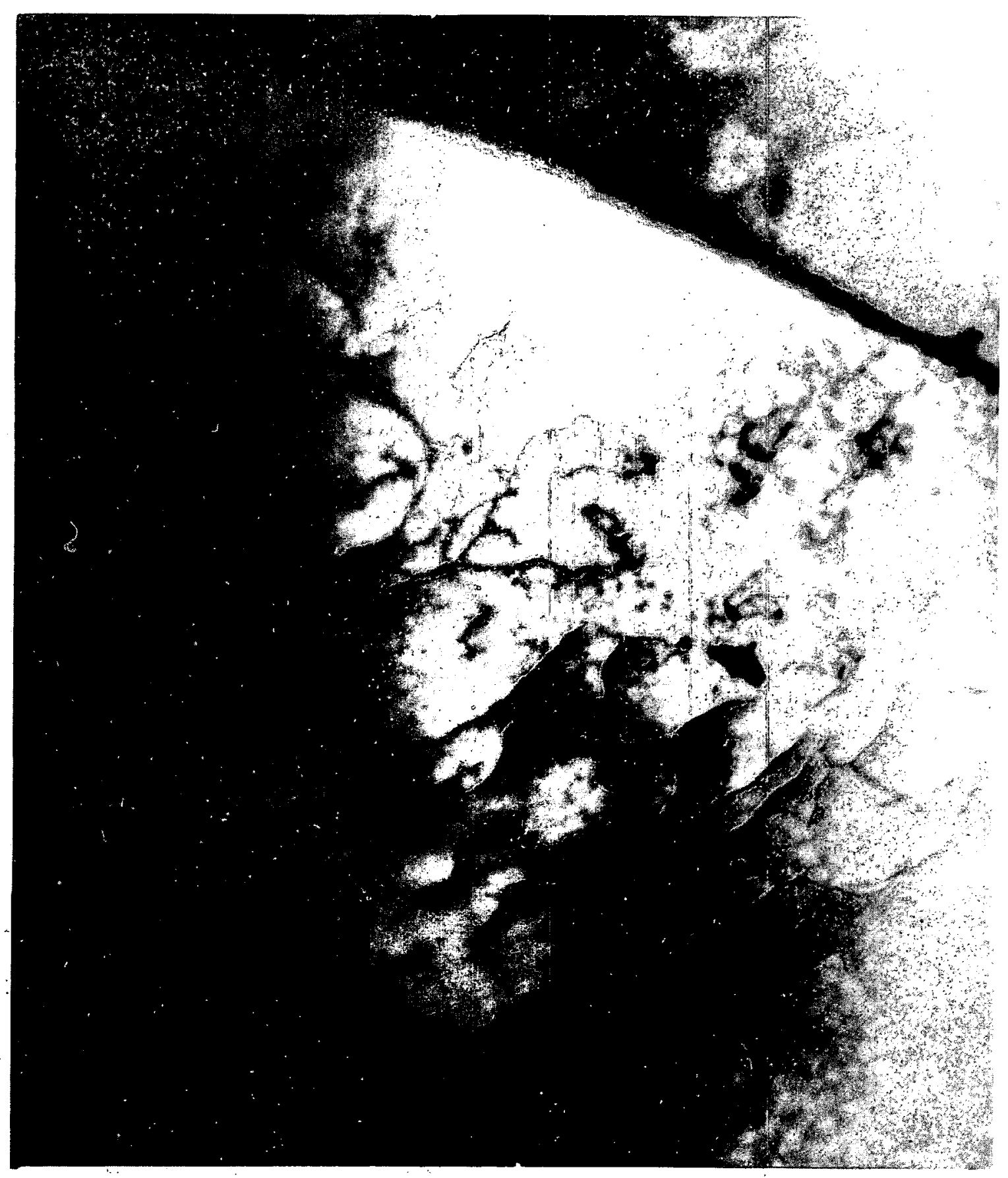

Fi gure 15. A Typical $\mathrm{T}$ win in the $300^{\circ} \mathrm{C}$ Tensile Specimen Occupies Nearly the Entire Field. Note the sparcity of dislocations in the twins. (Plate $775 ; 86,100 \mathrm{X}$ ) 


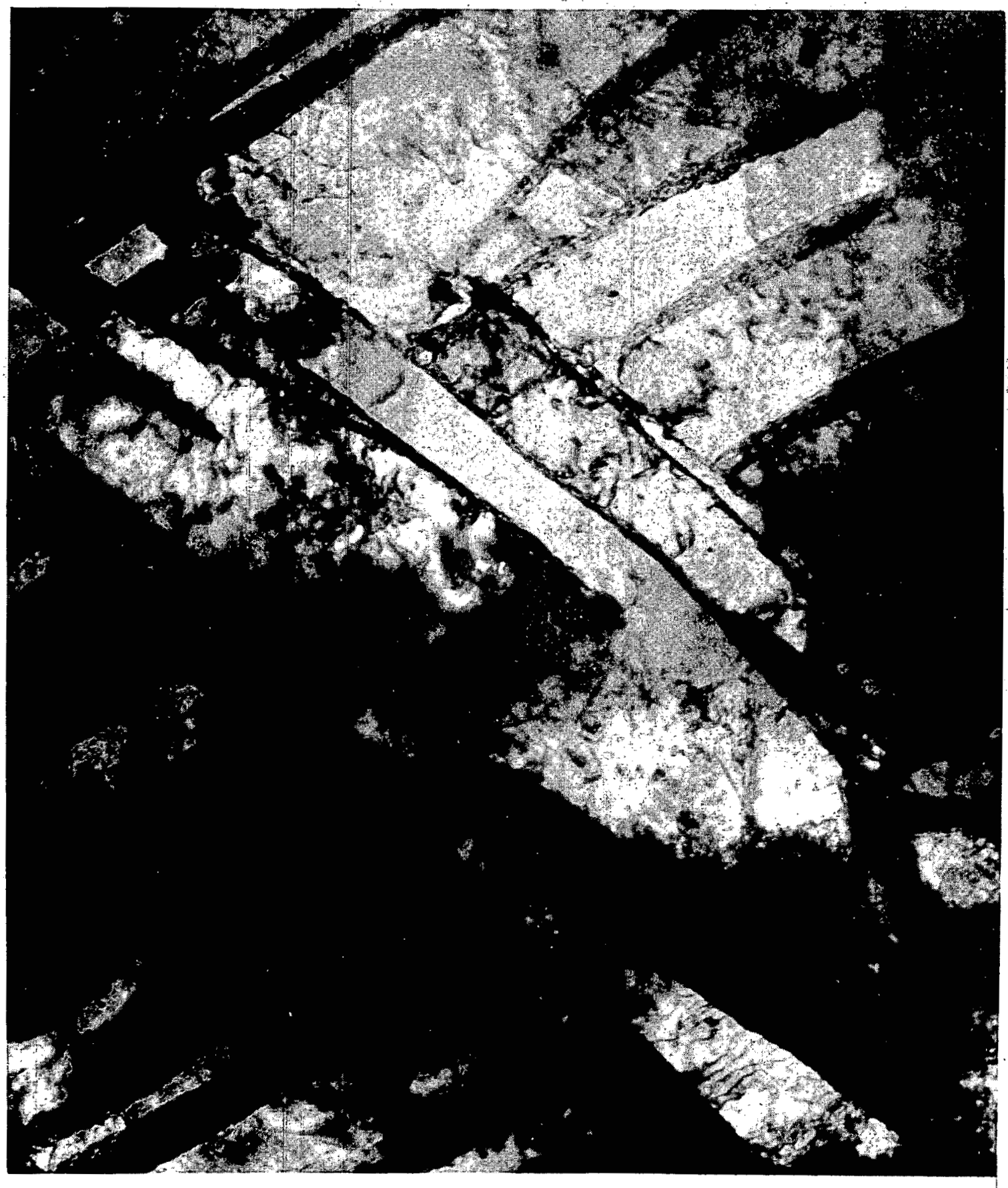

Figure 16. A Tensile Specimen of Uranium Pulled at $500^{\circ} \mathrm{C}$ Shown in

a Transverse Section Near the Break. The dislocation networks (network of lines) in the boundaries are between the slipped areas. (Plate 990; 86,100X) 


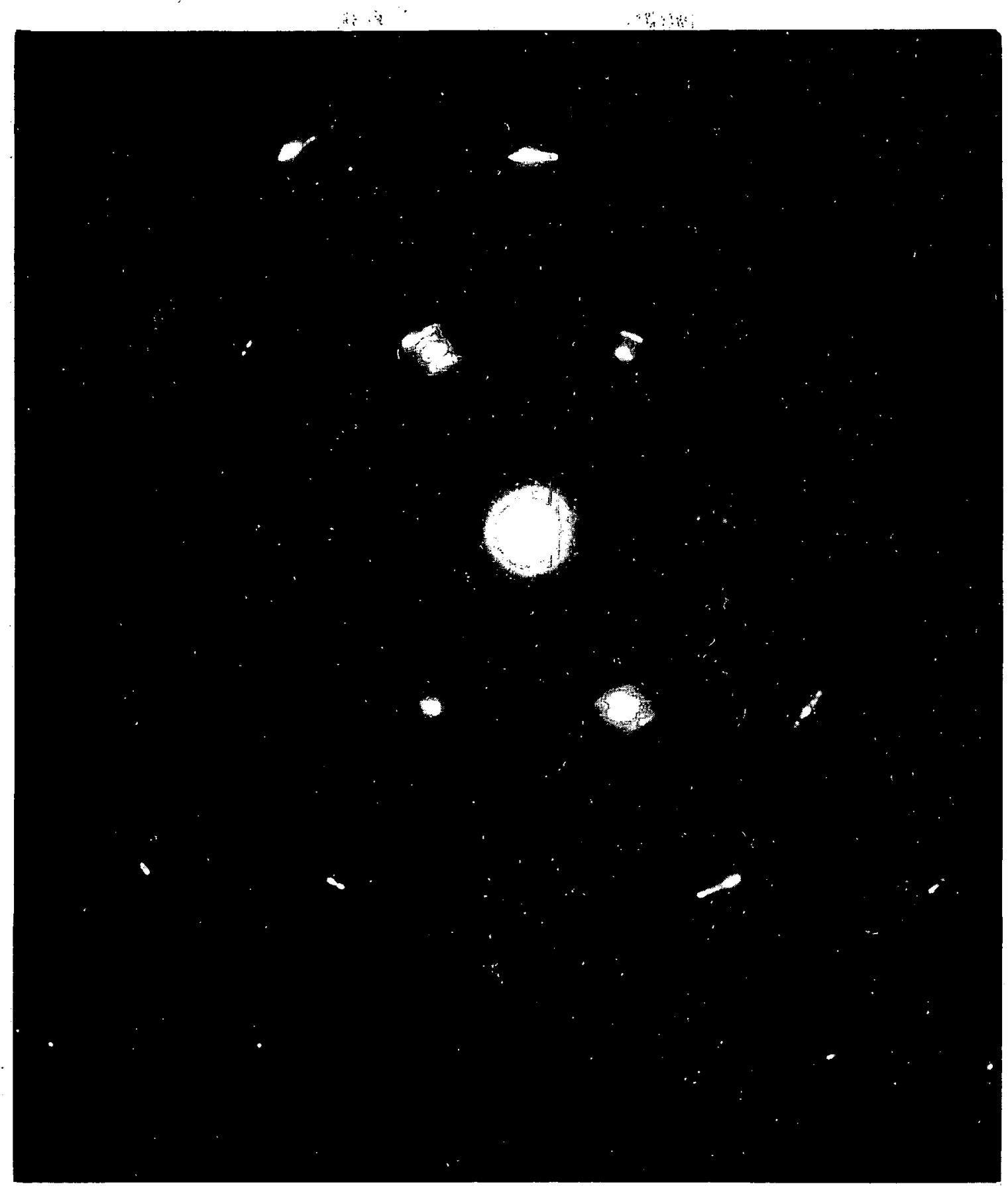

Figure 17. Diffraction Pattern from the Central Area of Figure 16.

The repeated patterns are slightly rotated from each other because of a slight reorientation of each slipped area. 